\title{
On the Language of Nested Tuple Generating Dependencies
}

\author{
PHOKION G. KOLAITIS, UC Santa Cruz and IBM Research - Almaden \\ REINHARD PICHLER, TU Wien \\ EMANUEL SALLINGER, TU Wien and University of Oxford \\ VADIM SAVENKOV, Vienna University of Economics and Business
}

\begin{abstract}
During the past 15 years, schema mappings have been extensively used in formalizing and studying such critical data interoperability tasks as data exchange and data integration. Much of the work has focused on GLAV mappings, i.e., schema mappings specified by source-to-target tuple-generating dependencies (s-t tgds), and on schema mappings specified by second-order tgds (SO tgds), which constitute the closure of GLAV mappings under composition. In addition, nested GLAV mappings have also been considered, i.e., schema mappings specified by nested tgds, which have expressive power intermediate between s-t tgds and SO tgds. Even though nested GLAV mappings have been used in data exchange systems, such as IBM's Clio, no systematic investigation of this class of schema mappings has been carried out so far. In this article, we embark on such an investigation by focusing on the basic reasoning tasks, algorithmic problems, and structural properties of nested GLAV mappings. One of our main results is the decidability of the implication problem for nested tgds. We also analyze the structure of the core of universal solutions with respect to nested GLAV mappings and develop useful tools for telling apart SO tgds from nested tgds. By discovering deeper structural properties of nested GLAV mappings, we show that also the following problem is decidable: Given a nested GLAV mapping, is it logically equivalent to a GLAV mapping?
\end{abstract}

CCS Concepts: • Theory of computation $\rightarrow$ Data exchange; $\bullet$ Information systems $\rightarrow$ Mediators and data integration;

Additional Key Words and Phrases: Schema mappings, data integration, data exchange, nested dependencies, second-order dependencies

The research of Reinhard Pichler, Emanuel Sallinger, and Vadim Savenkov was supported by the Austrian Science Fund, projects (FWF):P25207-N23 and (FWF):Y698, and the Vienna Science and Technology Fund, project ICT12-015. The research of Phokion Kolaitis on this article was partially supported by NSF Grant IIS-1217869. The full version was completed while Kolaitis was visiting the Simons Institute for the Theory of Computing during the fall of 2016. The research of Emanuel Sallinger was supported by the EPSRC programme grant EP/M025268/1, the Horizon 2020 grant 809965 and the Vienna Science and Technology Fund (WWTF) WWTF grant VRG18-013.

Authors' addresses: P. G. Kolaitis, University of California, Santa Cruz, Computer Science and Engineering Department, CA 95064, USA and IBM Research - Almaden, San Jose, CA 95120, USA; email: kolaitis@ucsc.edu; R. Pichler, TU Wien, Faculty of Informatics, Institute of Logic and Computation, Database and Artificial Intelligence Group, 1040 Vienna, Austria; email: pichler@dbai.tuwien.ac.at; E. Sallinger, TU Wien, Faculty of Informatics, Institute of Logic and Computation, Database and Artificial Intelligence Group, Knowledge Graph Lab, 1040 Vienna, Austria and University of Oxford, Department of Computer Science, OX1 3QD, Oxford, United Kingdom; email: sallinger@dbai.tuwien.ac.at; V. Savenkov, Vienna University of Economics and Business, Institute for Information Business, Welthandelsplatz 1, 1020 Vienna, Austria; email: vadim.savenkov@wu.ac.at.

Permission to make digital or hard copies of all or part of this work for personal or classroom use is granted without fee provided that copies are not made or distributed for profit or commercial advantage and that copies bear this notice and the full citation on the first page. Copyrights for components of this work owned by others than the author(s) must be honored. Abstracting with credit is permitted. To copy otherwise, or republish, to post on servers or to redistribute to lists, requires prior specific permission and/or a fee. Request permissions from permissions@acm.org.

(C) 2020 Copyright held by the owner/author(s). Publication rights licensed to ACM.

0362-5915/2020/07-ART8 \$15.00

https://doi.org/10.1145/3369554

ACM Transactions on Database Systems, Vol. 45, No. 2, Article 8. Publication date: July 2020. 


\section{ACM Reference format:}

Phokion G. Kolaitis, Reinhard Pichler, Emanuel Sallinger, and Vadim Savenkov. 2020. On the Language of Nested Tuple Generating Dependencies. ACM Trans. Database Syst. 45, 2, Article 8 (July 2020), 59 pages. https://doi.org/10.1145/3369554

\section{INTRODUCTION}

Schema mappings are high-level specifications, typically expressed in some logical formalism, that describe the relationship between two database schemas, called the source schema and the target schema. During the past 15 years, schema mappings have been extensively used in formalizing and studying such critical data interoperability tasks as data exchange and data integration. Much of the work has focused on two classes of schema mappings: GLAV mappings and mappings specified by SO tgds. A GLAV mapping is specified by a finite set of source-to-target tuple-generating dependencies (s-t tgds), which are first-order formulas of the form $\forall \vec{x}(\varphi(\vec{x}) \rightarrow \exists \vec{y} \psi(\vec{x}, \vec{y}))$ with $\varphi(\vec{x})$ a conjunction of atoms over the source schema and $\psi(\vec{x}, \vec{y})$ a conjunction of atoms over the target schema. As the name suggests, a second-order tuple-generating dependency (SO tgd) is a second-order formula; it starts with a string of existential function quantifiers that is followed by a conjunction of first-order formulas that resemble s-t tgds, but allow function terms in atomic formulas and also equalities between such terms. As shown in Reference [11], SO tgds are the right language for expressing compositions of GLAV schema mappings. The study of GLAV mappings and mappings specified by SO tgds has spanned a wide range of problems, from expressive power and algorithms to optimization and structural properties; for comprehensive overviews of the literature, see References [1, 19, 21, 25, 27].

In addition to GLAV mappings and mappings specified by SO tgds, two other classes of schema mappings of intermediate expressive power have also been considered. The first is the class of nested GLAV mappings that are specified by finitely many nested tgds, that is, first-order formulas that, informally, are obtained by a finite "nesting" of s-t tgds inside other s-t tgds. For example, the expression

$$
\forall x_{1} x_{2}\left(S\left(x_{1}, x_{2}\right) \rightarrow \exists y\left(R\left(y, x_{2}\right) \wedge \forall x_{3}\left(S\left(x_{1}, x_{3}\right) \rightarrow R\left(y, x_{3}\right)\right)\right)\right)
$$

is a nested tgd. The second is the class of plain SO tgds, which consists of those SO tgds that contain no nested terms (i.e., no functional terms that have other functional terms as arguments) and no equalities between terms. For example, the expression

$$
\exists f \forall x \forall y(S(x, y) \rightarrow R(f(x), f(y)))
$$

is a plain SO tgd. We now describe the different reasons and motivation that led to the introduction of these two classes of schema mappings.

Nested GLAV mappings were introduced in Reference [13] and demonstrated in Reference [18] as an enhancement of the specification language of the Clio system, which, at that time, was being developed at the IBM Almaden Research Center and later became part of IBM's InfoSphere BigInsights suite. Richer nested mappings, including nested XML mappings and nested secondorder tgds, were subsequently used in the Clip system [26]. In the peer data management context, in which data are exchanged between several different peer nodes (instead of between a single source schema and a single target schema), the Piazza system [16] was capable of performing query reformulation over XML data sources. As pointed out in Reference [6], the underlying mapping language Piazza XML (syntactically a fragment of XQuery) is essentially the language of nested tgds. Nested schema transformations in the peer data integration context were also used in the HePToX system [5]. 
The main argument in favor of nested GLAV mappings over GLAV mappings given in References $[13,18]$ is that they produce specifications that are more compact and also reflect more accurately the correlations between data; moreover, since they are specified in first-order logic, nested GLAV mappings give rise to transformations that, like those arising from GLAV mappings, can be implemented using SQL queries. Compared to SO tgds, nested GLAV mappings "offer a more natural programming paradigm for mapping tasks" [13]. This state of affairs suggests that there are situations in which it is important to determine if an SO tgd can be replaced by an equivalent nested GLAV mapping. Sufficient conditions under which such a rewriting is possible have been identified in Reference [4] and, very recently, in Reference [15].

Plain SO tgds were introduced and studied in depth in Reference [2] as a language particularly well suited for handling both composition and inversion of GLAV mappings. The results in Reference [2] make a strong case that plain SO tgds form the right language for handling CQcomposition and inversion of GLAV mappings, where CQ-composition is a variant of the composition operator in which two schema mappings are considered to be equivalent if they give rise to the same certain answers for conjunctive queries (the notion of CQ-composition was introduced in Reference [24]).

In terms of expressive power, nested GLAV mappings are strictly more expressive than GLAV mappings and strictly less expressive than mappings specified by plain SO tgds. As a matter of fact, it is known that the nested tgd given earlier is not logically equivalent to any finite set of s-t tgds, while the plain SO tgd given earlier is not logically equivalent to any nested GLAV mapping. Nested GLAV mappings and plain SO tgds share several desirable structural properties, such as admitting universal solutions and being closed under target homomorphisms [2,28]. These similarities notwithstanding, it should be kept in mind that nested tgds and SO tgds belong to intrinsically different logical formalisms (first-order logic vs. second-order logic), a fact that may translate to different algorithmic behavior. For instance, the data complexity of the model checking problem of nested tgds is in LOGSPACE, while the data complexity of plain SO tgds is NP-complete. As regards reasoning tasks, the decidability of the implication problem for plain SO tgds remains open, whereas the logical equivalence problem for SO tgds (hence, also the implication problem for SO tgds) is undecidable. In fact, there is no algorithm even for deciding whether a given SO tgd is logically equivalent to a given finite set of s-t tgds (see References [3, 12]). Coping with this complexity motivates the research on simplifying SO tgds and, in particular, plain SO tgds with first-order nested tgds [4, 15].

In spite of the uses and applications of nested tgds, no systematic investigation of nested tgds in their own right has been carried out to date. Our goal in this article is to embark on such an investigation by focusing on the basic reasoning tasks, algorithmic problems, and structural properties of nested GLAV mappings.

The implication problem is a fundamental problem in mathematical logic and also one of the central problems in database dependency theory. Our first main result is that the implication problem (and, hence, the equivalence problem) for nested tgds is decidable, in contrast to SO tgds. Our decision procedure for the implication problem for nested tgds is rather elaborate and entails a delicate analysis of the properties of the chase procedure for nested tgds.

After this, we address the problem of telling apart nested tgds from s-t tgds. To that end, we show that the following problem is decidable: Given a nested GLAV mapping, is it logically equivalent to some GLAV mapping? The situation is less clear regarding the problem of telling apart plain SO tgds from nested tgds. Indeed, at present, it is not known whether or not the following problem is decidable: Given a plain SO tgd, is it logically equivalent to some nested GLAV mapping? Even though we do not settle the decidability of this problem here, we succeed in providing useful and 
easy-to-use sufficient conditions for telling that a given plain SO tgd is not logically equivalent to a nested GLAV mapping.

The aforementioned algorithm for telling apart nested tgds from s-t tgds, as well as the aforementioned sufficient conditions for telling apart a plain SO tgd from nested tgds, are derived by discovering new properties of the structure of the cores of universal solutions with respect to nested GLAV mappings. We believe that these properties are of interest in their own right and may play a role in structural characterizations of schema-mapping languages.

Finally, we study settings where key dependencies or, more generally, equality generating dependencies (egds) over the source schema are present. By revisiting the fundamental decision problems of logical equivalence and of telling apart schema mappings in different formalisms, we unveil further significant differences between nested tgds and plain SO tgds. In Reference [12], the logical equivalence problem for plain SO tgds was shown undecidable if the source schema contains key dependencies. In contrast, here, we show that the implication problem (and, hence, the logical equivalence problem) for nested tgds remains decidable even in the presence of arbitrary source egds. Likewise, we show that the problem of deciding if a given nested GLAV mapping is logically equivalent to some GLAV mapping remains decidable if arbitrary source egds are allowed. Again, this is in sharp contrast to plain SO tgds, for which we prove undecidability of the following problems in the presence of source key dependencies: Given a plain SO tgd, is it logically equivalent to a GLAV mapping (or to a nested GLAV mapping, respectively)?

One of the chief goals in data exchange and data integration is to perform query answering. Hence, in addition to logical equivalence between mappings, one is also interested in $C Q-$ equivalence. By definition, two mappings are CQ-equivalent if for every target conjunctive query $q$, the certain answers of $q$ are the same for the two mappings. In general, logical equivalence implies CQ-equivalence, but not vice versa. However, logical equivalence and CQ-equivalence coincide for plain SO tgds and, in particular, for nested GLAV mappings. This can be seen by combining results from References [2] and [9]. More precisely, in Proposition 3.14 of Reference [9] the following property is shown: Let $\mathcal{M}$ and $\mathcal{M}^{\prime}$ be schema mappings such that both are preserved under target homomorphisms and both have the following property: For every instance I, if there is a solution for $I$, then there is a universal solution for $I$. Then, the fact that $\mathcal{M}$ and $\mathcal{M}^{\prime}$ are CQ-equivalent implies that $\mathcal{M}$ and $\mathcal{M}^{\prime}$ are logically equivalent. Moreover, in Theorem 14 of Reference [2] it is shown that plain SO tgds possess these properties. Thus, even though we state and prove our results for logical equivalence only, they also hold for CQ-equivalence as well.

The remainder of the article is organized as follows: Section 2 contains the definitions of the basic concepts and background material. Section 3 is devoted to the implication problem for nested tgds. Section 4 contains the analysis of the core of the universal solutions with respect to nested GLAV mappings, and the applications of this analysis to differentiating nested tgds from $s-t$ tgds as well as plain SO tgds from nested tgds. Section 5 revisits the problems studied in earlier sections when source key constraints are also present in the specification of the schema mappings at hand. The article concludes with a discussion of open problems and directions for future research.

Relationship to previous work. This article is the full version of the conference paper [20]. This full version represents a significant expansion over the conference proceedings version. In particular, the body of the article includes detailed proofs of some of the main results and additional explanations. In addition, the present article contains an Appendix featuring some of the more technical proofs and a number of figures that facilitate the understanding of these proofs. In total, this submission contains roughly double the amount of material compared to the conference paper. 


\section{PRELIMINARIES}

Schemas, Instances, and Homomorphisms. A schema $\mathrm{R}$ is a finite set $\left\{R_{1}, \ldots, R_{k}\right\}$ of relation symbols, where each $R_{i}$ has a fixed arity $\geq 1$. An instance $I$ over $\mathbf{R}$, or an $\mathbf{R}$-instance, is a set $R_{1}^{I} \cup \ldots \cup R_{k}^{I}$, where each $R_{i}^{I}$ is a finite relation of the same arity as $R_{i}$. We will often use $R_{i}$ to denote both the relation symbol and the relation $R_{i}^{I}$ that instantiates it, when instance $I$ is clear from the context.

The active domain dom $(I)$ of an instance $I$ is the set of values occurring in the relations of $I$. We assume these values to be of two sorts, namely, constants and (labeled) nulls. By CONST and NULLS, we denote the disjoint sets of all constants and all nulls, respectively. Intuitively, nulls are used as placeholders for unknown constant values. We write const $(I)$ and nulls $(I)$ to denote, respectively, the set of constants and the set of nulls in dom(I). A fact of an instance $I$ over a schema $\mathbf{R}$ is a tuple occurring in one of the relations $R_{i}$ of $I$. Such a fact is denoted $R_{i}\left(v_{1}, \ldots, v_{m}\right)$ or, for short, $R(\vec{a})$. Here, $\vec{a}=\left(v_{1}, \ldots, v_{m}\right)$ is a tuple of values from $\operatorname{dom}(I)$, every $v_{i}$ being either a constant or a null. The empty tuple is denoted by ().

Let $\mathrm{S}$ and $\mathrm{T}$ be two schemas with no relation symbols in common. We refer to $\mathrm{S}$ as the source schema, and $\mathrm{T}$ as the target schema. Similarly, we refer to $\mathrm{S}$-instances as source instances, and Tinstances as target instances. We assume that the active domains of source instances consist of constants; the active domains of target instances may consist of constants and nulls. Note that variables (in logical formulas) should not be confused with nulls (in target instances).

Let $J$ be a target instance. The Gaifman graph of facts of $J$ is the graph whose nodes are the facts of $J$ and there is an edge between any two distinct facts if they have a null in common. We say that a target instance $J$ is connected if the Gaifman graph of facts of $J$ is connected. A fact block (f-block) of $J$ is a connected component of the Gaifman graph of facts of $J$. The fact block size (f-block size) of $J$ is the cardinality of the largest f-block of $J$.

Let $J_{1}$ and $J_{2}$ be two target instances. A function $h$ that maps constants and nulls to constants and nulls is a homomorphism from $J_{1}$ to $J_{2}$ if the following holds: (i) for every constant $c$, we have that $h(c)=c$; and (ii) for every relation symbol $R$ in $\mathrm{R}$ and every tuple $\left(a_{1}, \ldots, a_{n}\right) \in R^{J_{1}}$, we have that $\left(h\left(a_{1}\right), \ldots, h\left(a_{n}\right)\right) \in R^{J_{2}}$. We use the notation $J_{1} \rightarrow J_{2}$ to denote that there is a homomorphism from $J_{1}$ to $J_{2}$. We say that $J_{1}$ is homomorphically equivalent to $J_{2}$, written $J_{1} \leftrightarrow J_{2}$, if $J_{1} \rightarrow J_{2}$ and $J_{2} \rightarrow J_{1}$. The core of an instance $J$, denoted core $(J)$, is the smallest subinstance of $J$ that is homomorphically equivalent to $J$. If there are multiple cores of $J$, then they are all isomorphic [17]. A homomorphism from $J$ to $J$ is called an endomorphism. An endomorphism is called proper if $h(v) \in$ CONST holds for at least one null $v$ or $h\left(v_{1}\right)=h\left(v_{2}\right)$ holds for two distinct nulls $v_{1}, v_{2}$.

Example 2.1. Consider the following instance $I_{1}=C_{1} \cup C_{2}$ such that $C_{1}=\left\{A\left(u_{1}, u_{2}\right), A\left(u_{2}, u_{3}\right)\right\}$ with nulls $u_{i}$ and $C_{2}=\left\{A\left(v_{1}, v_{2}\right), A\left(v_{2}, v_{3}\right), A\left(v_{3}, v_{1}\right)\right\}$ with nulls $v_{j}$. Intuitively, $I_{1}$ constitutes a directed graph where $A$ is the edge relation and the nulls $u_{i}$ and $v_{j}$ are the vertices. We see that the smaller subinstance (i.e., subgraph) $C_{1}$ can be homomorphically mapped to $C_{2}$. This is witnessed by the homomorphism that maps each $u_{i}$ to the respective $v_{i}$. Thus, we have that core $\left(I_{1}\right)=C_{2}$, as $C_{2}$ is homomorphically equivalent to the entire instance $I_{1}$.

The core looks quite different if we add a single "self-loop" (i.e., a fact $A(w, w)$ for some null $w$ ) to the instance. More precisely, let $I_{2}=I_{1} \cup S$ with $S=\{A(w, w)\}$ where $w$ is a null. Then both $C_{1}$ and $C_{2}$ can be homomorphically mapped to $S$, that is, core $\left(I_{2}\right)=S$. In fact, it is a general observation that arbitrary graphs containing a self-loop "collapse" to the self-loop in the core.

Schema mappings. A schema mapping is a triple $\mathcal{M}=(\mathrm{S}, \mathrm{T}, \Sigma)$, where $\mathrm{S}$ is the source schema, $\mathrm{T}$ is the target schema, and $\Sigma$ is a set of constraints, which are logical formulas describing the relationship between $\mathrm{S}$ and $\mathrm{T}$. We say that $\mathcal{M}$ is specified by $\Sigma$; often, we will identify the set $\Sigma$ of constraints with the mapping $\mathcal{M}$. 
If $I$ is a source instance and $J$ is a target instance such that the pair $(I, J)$ satisfies $\Sigma$ (written $(I, J) \models \Sigma)$, then we say that $J$ is a solution of $I$ w.r.t. $\mathcal{M}$. We say that $J$ is a universal solution for $I$ w.r.t. $\mathcal{M}$ if $J$ is a solution for $I$ and for every solution $J^{\prime}$ for $I$, we have $J \rightarrow J^{\prime}$. If $C$ is a class of schema mappings, we say that $C$ admits universal solutions if for every schema mapping $\mathcal{M}$ in $C$ and every source instance $I$, a universal solution for $I$ w.r.t. $\mathcal{M}$ exists.

s-t tgds. A source-to-target tuple-generating dependency (in short, s-t tgd) is a first-order sentence of the form $\forall \vec{x}(\varphi(\vec{x}) \rightarrow \exists \vec{y} \psi(\vec{x}, \vec{y}))$, where $\varphi(\vec{x})$ is a conjunction of atoms over S, each variable in $\vec{x}$ occurs in at least one atom in $\varphi(\vec{x})$, and $\psi(\vec{x}, \vec{y})$ is a conjunction of atoms over $\mathrm{T}$ with variables in $\vec{x}$ and $\vec{y}$. For simplicity, we will often suppress writing the universal quantifiers $\forall \vec{x}$ in the above formula. We refer to $\varphi(\vec{x})$ as the left-hand side, or antecedent, and $\exists \vec{y} \psi(\vec{x}, \vec{y})$ as the right-hand side, or conclusion.

Another name for s-t tgds is global-and-local-as-view (GLAV) constraints (see Reference [22]). We refer to a schema mapping specified entirely by a finite set of GLAV constraints as a GLAV mapping.

As shown in Reference [8], the class of GLAV mappings admits universal solutions. Moreover, if $\mathcal{M}$ is a GLAV mapping, then given a source instance $I$, the so-called "canonical" universal solution chase $(I, \mathcal{M})$ can be produced via the oblivious chase procedure, defined next.

Let $\forall \vec{x}(\varphi(\vec{x}) \rightarrow \exists \vec{y} \psi(\vec{x}, \vec{y}))$ be an s-t tgd in $\mathcal{M}$. For a conjunction of atoms $\varphi(\vec{x})$, we use $\vec{a}$ to define an assignment for $\vec{x}$ consisting of constants, such that the $i$ th element of $\vec{a}$ replaces the $i$ th variable of $\vec{x}$ in $\varphi(\vec{x})$, resulting in the formula $\varphi(\vec{a})$. We write $I=\varphi(\vec{x})$ if there exists an assignment $\vec{a}$ for $\vec{x}$ such that for each atom $R\left(a_{1}, \ldots, a_{k}\right)$ of $\varphi(\vec{a})$, the fact $\left(a_{1}, \ldots, a_{k}\right)$ is in $R^{I}$. Such an assignment $\vec{a}$ is called a satisfying assignment for $\varphi(\vec{x})$ w.r.t. $I$ (we omit the phrase "w.r.t. $I$ " if $I$ is clear from the context): this is denoted as $I=\varphi(\vec{a})$. For every satisfying assignment $\vec{a}$ for $\varphi(\vec{x})$ w.r.t. $I$, the oblivious chase adds to the instance chase $(I, \mathcal{M})$ all atoms of $\psi(\vec{a}, \vec{B})$ as facts, where $\vec{B}$ is an assignment replacing all variables $y$ in $\vec{y}$ with fresh, pairwise-distinct nulls. Such an extension of chase $(I, \mathcal{M})$ is performed for each satisfying assignment of each s-t $\operatorname{tgd}$ in $\mathcal{M}$.

egds. An equality generating dependency (egd) has the form $\forall \vec{x}\left(\varphi(\vec{x}) \rightarrow x_{i}=x_{j}\right)$, where the antecedent $\varphi(\vec{x})$ is a conjunction of atoms and $x_{i}, x_{j}$ are the $i$ th and the $j$ th elements of $\vec{x}$, respectively. Sets of egds generalize functional dependencies. The chase of an instance $I$ with the above egd proceeds as follows: Whenever $\vec{a}$ is a satisfying assignment for $\varphi(\vec{x})$ with $a_{i} \neq a_{j}$, then the chase replaces in $I$ each occurrence of $a_{i}$ with $a_{j}$. Typically in data exchange, egds on the target schema are defined, and only those unifications are allowed where at least one value of $a_{i}, a_{j}$ is a null: If $a_{i}$ and $a_{j}$ are distinct constants, then the chase halts with failure. This convention does not apply here, since we use egds to specify admissible source instances.

SO tgds and Plain SO tgds. Second-Order tgds, or SO tgds, were introduced in Reference [11], where it was shown that SO tgds are exactly the dependencies needed to specify the composition of an arbitrary number of GLAV mappings. Before we formally define SO tgds, we need to define terms. Given collections $\vec{x}$ of variables and $\vec{f}$ of function symbols, a term (based on $\vec{x}$ and $\vec{f}$ ) is defined recursively as follows: (1) Every variable in $\vec{x}$ is a term; (2) If $f$ is a $k$-ary function symbol in $\vec{f}$ and $t_{1}, \ldots, t_{k}$ are terms, then $f\left(t_{1}, \ldots, t_{k}\right)$ is a term.

Let $\mathrm{S}$ be a source schema and $\mathrm{T}$ a target schema. A second-order tuple-generating dependency (SO $\operatorname{tg} d)$ is a formula of the form:

$$
\exists \vec{f}\left(\left(\forall \vec{x}_{1}\left(\varphi_{1} \rightarrow \psi_{1}\right)\right) \wedge \cdots \wedge\left(\forall \vec{x}_{n}\left(\varphi_{n} \rightarrow \psi_{n}\right)\right)\right), \text { where }
$$

(1) $\vec{f}$ is a sequence of function symbols. 
(2) Each $\varphi_{i}$ is a conjunction of (i) relational atoms $S\left(y_{1}, \ldots, y_{k}\right)$, where $S$ is a $k$-ary relation symbol of schema $\mathrm{S}$ and $y_{1}, \ldots, y_{k}$ are variables in $\vec{x}_{i}$, not necessarily distinct, and (ii) equalities of the form $t=t^{\prime}$ where $t$ and $t^{\prime}$ are terms based on $\vec{x}_{i}$ and $\vec{f}$.

(3) Each $\psi_{i}$ is a conjunction of atoms $T\left(t_{1}, \ldots, t_{l}\right)$, where $T$ is an $l$-ary relation symbol of schema $\mathrm{T}$ and $t_{1}, \ldots, t_{l}$ are terms based on $\vec{x}_{i}$ and $\vec{f}$.

(4) Each variable in $\vec{x}_{i}$ appears in some relational atom of $\varphi_{i}$.

As an example, the formula

$$
\begin{aligned}
\exists f(\forall e(E m p(e) & \rightarrow \operatorname{Mgr}(e, f(e))) \wedge \\
\forall e(\operatorname{Emp}(e) & \wedge(e=f(e)) \rightarrow \operatorname{SelfMgr}(e)))
\end{aligned}
$$

expresses the property that every employee has a manager, and if an employee is the manager of himself/herself, then this employee is a self-manager.

Note that SO tgds allow for nested terms and for equalities between terms. A nested term is a functional term that contains a functional term as an argument. A plain SO tgd is an SO tgd that contains no nested terms and no equalities. For example, the preceding $\mathrm{SO}$ tgd is not plain, while the following SO tgd is plain:

$$
\exists f \forall x \forall y(S(x, y) \rightarrow R(f(x), f(y))) .
$$

The properties of plain SO tgds were recently investigated in Reference [2]. It is easy to see that every GLAV schema mapping is logically equivalent to a plain SO tgd. Moreover, as shown in Reference [11], the class of SO tgds admits universal solutions, hence, the same holds true for the class of plain SO tgds. In fact, the oblivious chase procedure can be extended to SO tgds, so that if $\sigma$ is an SO $\operatorname{tgd}$ and $I$ is a source instance, then chase $(I, \sigma)$ is the canonical universal solution for $I$ w.r.t. $\sigma$.

In what follows, we will often suppress writing the existential second-order quantifiers and the universal first-order quantifiers in front of SO tgds.

Nested tgds. Let $X$ and $Y$ be disjoint sets of variables. Consider the following recursive syntax rule:

$$
\chi:=\forall \vec{x}\left(\beta_{1} \wedge \ldots \wedge \beta_{k} \rightarrow \exists \vec{y}\left(\alpha_{1} \wedge \ldots \wedge \alpha_{n} \wedge \chi_{1} \wedge \ldots \wedge \chi_{\ell}\right)\right),
$$

where (i) $n, \ell$ are nonnegative integers, (ii) $\beta_{1}, \ldots, \beta_{k}$ with $k \geq 1$ are constant-free atoms whose relation names belong to the source schema, (iii) $\alpha_{1}, \ldots, \alpha_{n}$ are constant-free atoms whose relation names belong to the target schema, (iv) every variable that occurs in $\vec{x}$ also occurs in some atom among $\beta_{1}, \ldots, \beta_{k}$, (v) every variable occurring in some atom in $\beta_{1}, \ldots, \beta_{k}$ also belongs to $X$; and (vi) every variable that occurs in $\vec{y}$ belongs to $Y$.

An empty conjunction is understood to be equivalent to true. In particular, if $\ell=0$, then $\chi_{1} \wedge$ $\ldots \wedge \chi_{\ell} \equiv$ true, which is the base case of the recursion.

A nested $\operatorname{tg} d$ is a first-order sentence that can be generated by the recursive syntax rule (1). It can be assumed w.l.o.g. that every variable that occurs in a nested tgd is quantified exactly once by either an existential or universal quantifier. By subformula $\tau$ of a nested tgd, we will always denote an implication $\forall \vec{x}\left(\varphi\left(\vec{x}_{0}, \vec{x}\right) \rightarrow \exists \vec{y} \psi\left(\vec{x}_{0}, \vec{x}, \vec{y}_{0}, \vec{y}\right)\right)$ generated by one of the symbols $\chi_{1} \ldots \chi_{\ell}$ in the syntax rule (1). Here, $\vec{x}_{0}$ and $\vec{y}_{0}$ are tuples of free variables, respectively, universally and existentially quantified outside the subformula in the nested tgd. For instance, the conclusion $\psi\left(\vec{x}_{0}, \vec{x}, \vec{y}_{0}, \vec{y}\right)$ of $\tau$ may contain a conjunct $\tau^{\prime}=\forall \vec{x}^{\prime}\left(\varphi^{\prime}\left(\vec{x}_{0}^{\prime}, \vec{x}^{\prime}\right) \rightarrow \exists \vec{y}^{\prime} \psi^{\prime}\left(\vec{x}_{0}^{\prime}, \vec{x}^{\prime}, \vec{y}_{0}^{\prime}, \vec{y}^{\prime}\right)\right)$ where $\vec{x}_{0}^{\prime}$ is a tuple of free variables containing both the free variables $\vec{x}_{0}$ of $\tau$ and the universally quantified variables $\vec{x}$ of $\tau$. Our convention is that variables in such a tuple $\vec{x}_{0}^{\prime}$ follow the order in which they are quantified in the nested tgd, that is, $\vec{x}_{0}^{\prime}$ is the concatenation $\vec{x}_{0} \cdot \vec{x}$. 


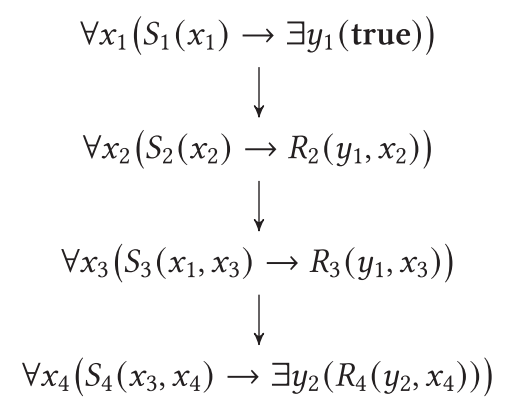

Fig. 1. Tree representation of $\sigma$.

Generating a nested $\operatorname{tgd} \sigma$ by the recursive syntax rule (1) yields a tree representation of $\sigma$, such that the root node of this tree is labeled by the subformula stemming from instantiating $\forall \vec{x}\left(\beta_{1} \wedge \ldots \wedge \beta_{k} \rightarrow \exists \vec{y}\left(\alpha_{1} \wedge \ldots \wedge \alpha_{n}\right)\right)$. Moreover, the root has $\ell$ subtrees, which correspond to the subformulas generated by $\chi_{1}, \ldots \chi \chi_{\ell}$. We refer to the subformulas labeling the nodes in this tree representation as parts of $\sigma$. The child, parent, ancestor, and descendant relations of nodes in the tree naturally carry over to the parts of $\sigma$. For a part $\sigma_{i}$ of $\sigma$, we thus write parent $\left(\sigma_{i}\right)$ to denote the parent $\sigma_{j}$ of $\sigma_{i}$ (i.e., $\sigma_{j}$ is the label of the parent node of the node labeled by $\sigma_{i}$ ) and we write $\operatorname{child}\left(\sigma_{i}\right)$, anc $\left(\sigma_{i}\right)$, and $\operatorname{desc}\left(\sigma_{i}\right)$ for the set of children, ancestors, and descendants, respectively, of $\sigma_{i}$. The part labeling the root node will be referred to as the root part of $\sigma$. Every part of $\sigma$ will be assumed to be uniquely identified by a positive integer index. The notation $\sigma_{i}\left(\vec{x}_{i}\right)$ will be used to explicitly specify the free variables $\vec{x}_{i}$ of the part $\sigma_{i}$. These are the variables quantified in anc $\left(\sigma_{i}\right)$.

Example 2.2. Assume that the nested $\operatorname{tgd} \sigma$ has the following form:

$$
\begin{aligned}
\forall x_{1}\left(S_{1}\left(x_{1}\right) \rightarrow \exists y_{1}(\right. & \forall x_{2}\left(S_{2}\left(x_{2}\right) \rightarrow\right. \\
\forall x_{3}\left(S_{3}\left(x_{1}, x_{3}\right)\right. & \rightarrow\left(R_{3}\left(y_{1}, x_{3}\right) \wedge\right. \\
\forall & \left.\left.\left.\left.x_{4}\left(S_{4}\left(x_{3}, x_{4}\right) \rightarrow \exists y_{2} R_{4}\left(y_{2}, x_{4}\right)\right)\right)\right)\right)\right) .
\end{aligned}
$$

There are four parts in $\sigma$ (see Figure 1 for a tree representation of $\sigma$ ):

- $\sigma_{0}: \forall x_{1}\left(S_{1}\left(x_{1}\right) \rightarrow \exists y_{1}(\right.$ true $\left.)\right)$

- $\sigma_{1}\left(x_{1}, y_{1}\right): \forall x_{2}\left(S_{2}\left(x_{2}\right) \rightarrow R_{2}\left(y_{1}, x_{2}\right)\right)$

- $\sigma_{2}\left(x_{1}, y_{1}\right): \forall x_{3}\left(S_{3}\left(x_{1}, x_{3}\right) \rightarrow R_{3}\left(y_{1}, x_{3}\right)\right)$

- $\sigma_{3}\left(x_{1}, x_{3}, y_{1}\right): \forall x_{4}\left(S_{4}\left(x_{3}, x_{4}\right) \rightarrow \exists y_{2}\left(R_{4}\left(y_{2}, x_{4}\right)\right)\right)$

Skolemization. In the above examples, parts of a nested tgd contain free variables that correspond to the existentially quantified variables of $\sigma$. More convenient is the Skolemized form of the nested tgd, in which free variables of the parts correspond to the universally quantified variables of $\sigma$. We use the standard first-order Skolemization procedure. Let SK denote a set of Skolem functional symbols disjoint from the set of variables, CONST and NULLS. These function symbols are considered as existentially quantified. The Skolemization replaces each existentially quantified variable $y$ in $\sigma$ with a unique term $f(\vec{x})$, where $f \in$ SK and $\vec{x}$ is the tuple of those universally quantified variables that occur in the same part as $\exists y$ or in some ancestor thereof. For a part $\sigma_{i}$, the set $\operatorname{vars}\left(\sigma_{i}\right)$ contains all Skolem terms replacing variables that were existentially quantified in $\sigma_{i}$ before Skolemization. 
Example 2.2 (continued). The Skolemization of the nested $\operatorname{tgd} \sigma$ is:

$$
\begin{aligned}
& \forall x_{1}\left(S_{1}\left(x_{1}\right) \rightarrow\right.\left(\forall x _ { 2 } \left(S_{2}\left(x_{2}\right) \rightarrow\right.\right. \\
&\left.\forall x_{2}\left(f_{1}\left(x_{1}\right), x_{2}\right)\right) \wedge \\
& \forall S_{3}\left(x_{1}, x_{3}\right) \rightarrow\left(R_{3}\left(f_{1}\left(x_{1}\right), x_{3}\right) \wedge\right. \\
&\left.\left.\left.\left.\forall x_{4}\left(S_{4}\left(x_{3}, x_{4}\right) \rightarrow R_{4}\left(f_{2}\left(x_{1}, x_{3}, x_{4}\right), x_{4}\right)\right)\right)\right)\right)\right) .
\end{aligned}
$$

The parts of $\sigma$ after Skolemization are given below.

- $\sigma_{0}=\forall x_{1}\left(S_{1}\left(x_{1}\right) \rightarrow\right.$ (true) $) ; \operatorname{vars}\left(\sigma_{0}\right)=\left\{f_{1}\left(x_{1}\right)\right\}$,

- $\sigma_{1}\left(x_{1}\right)=\forall x_{2}\left(S_{2}\left(x_{2}\right) \rightarrow R_{2}\left(f_{1}\left(x_{1}\right), x_{2}\right)\right) ; \operatorname{vars}\left(\sigma_{1}\right)=\emptyset$,

- $\sigma_{2}\left(x_{1}\right)=\forall x_{3}\left(S_{3}\left(x_{1}, x_{3}\right) \rightarrow R_{3}\left(f_{1}\left(x_{1}\right), x_{3}\right)\right) ; \operatorname{vars}\left(\sigma_{2}\right)=\emptyset$,

- $\sigma_{3}\left(x_{1}, x_{3}\right)=\forall x_{4}\left(S_{4}\left(x_{3}, x_{4}\right) \rightarrow R_{4}\left(f_{2}\left(x_{1}, x_{3}, x_{4}\right), x_{4}\right)\right)$; $\operatorname{vars}\left(\sigma_{3}\right)=\left\{f_{2}\left(x_{1}, x_{3}, x_{4}\right)\right\}$.

From now on, we only consider Skolemized tgds unless explicitly specified otherwise. As has become apparent in the previous example, after Skolemization, the parts no longer contain as free variables the existentially quantified variables of ancestor parts. We conclude this section by drawing a connection between nested tgds and SO tgds. Although syntactically, nested tgds differ from SO tgds, which do not have nested implications, a nested tgd can be converted into an SO $\operatorname{tgd}$.

E.g., a conversion procedure in Reference [14] first performs a Skolemization and then extends the antecedent in each subformula $\sigma_{i}$ generated by the symbol $\chi_{i}$ in rule (1) by adding to it the implication antecedents of all ancestor subformulas of $\sigma_{i}$ as conjuncts. A nested tgd can be shown equivalent to the resulting conjunction of its Skolemized parts, which is syntactically an SO tgd.

A nested $G L A V$ mapping is a schema mapping $\mathcal{M}=(\mathrm{S}, \mathrm{T}, \Sigma)$, where $\Sigma$ is a finite set of nested tgds. Unless mentioned otherwise, in this article, we will always assume dependencies (tgds, nested tgds, and SO tgds) to be source-to-target dependencies.

\section{THE IMPLICATION PROBLEM}

Let $\mathrm{S}$, $\mathrm{T}$ be two relational schemas and $\mathcal{M}=(\mathrm{S}, \mathrm{T}, \Sigma), \mathcal{M}^{\prime}=\left(\mathrm{S}, \mathrm{T}, \Sigma^{\prime}\right)$ be schema mappings, where $\Sigma$ and $\Sigma^{\prime}$ are expressed in some logical formalism (e.g., SO tgds or nested tgds). We say that $\Sigma$ implies $\Sigma^{\prime}$, denoted by $\Sigma \models \Sigma^{\prime}$, if for every instance $I$ of S and every instance $J$ of T such that $(I, J) \mid=\Sigma$, we have that $(I, J) \models \Sigma^{\prime}$. The implication problem asks: Given two finite sets $\Sigma$ and $\Sigma^{\prime}$ of constraints, does $\Sigma=\Sigma^{\prime}$ hold? Analogously, the (logical) equivalence problem asks if $\Sigma \equiv \Sigma^{\prime}$ holds, i.e., if $\Sigma$ and $\Sigma^{\prime}$ are satisfied by exactly the same pairs $(I, J)$ of source and target instances. In Section 5, we will study the implication and equivalence problems of schemas in the presence of a set $\Sigma_{s}$ of source constraints. In this case, only those source instances $I$ are considered that actually satisfy $\Sigma_{s}$. For instance, we will then say that $\Sigma$ implies $\Sigma^{\prime}$ if for every instance $I$ of S with $I=\Sigma_{s}$ and every instance $J$ of T such that $(I, J) \mid=\Sigma$, we have that $(I, J) \models \Sigma^{\prime}$.

Note that, since all instances considered are finite, this is the implication (and equivalence) problem in the finite. The main result of this section is as follows:

THEOREM 3.1. The implication problem for nested tgds is decidable.

We start by giving an informal, high-level explanation of the implication algorithm. All concepts used in this explanation (such as the chase with nested tgds) will be made precise below. Let $\sigma$ be a nested $\operatorname{tgd}$ and $\Sigma$ be a set of nested tgds. The proof of Theorem 3.1 will establish that if $\Sigma \vDash \sigma$, then there exists a pair of "canonical" source and target instances $\left(I_{p}, J_{p}\right)$ that satisfies $\sigma$ such that there is no homomorphism from $J_{p}$ to $\operatorname{chase}\left(I_{p}, \Sigma\right)$. The pair of instances $\left(I_{p}\right.$, chase $\left.\left(I_{p}, \Sigma\right)\right)$ then satisfies $\Sigma$ and falsifies $\sigma$, witnessing $\Sigma \not \sigma$. The canonical instances are constructed as minimal and in a sense most general instances enabling a chase of $I_{p}$ with $\sigma$, such that the number of triggerings 
of each part of $\sigma$ is bounded and the pattern of the chase has a certain form. This bound and the number of relevant chase patterns will appear quite high (in fact, non-elementary in the size of $\sigma$ and $\Sigma$ ). In Section 4 , we will show that this limit exhibits a crucial and so far unexplored difference between nested tgds and SO tgds.

The decision procedure behind Theorem 3.1 requires the introduction of several notions and technical tools that are presented in what follows:

Chase Forest. The oblivious chase of an instance with a nested tgd can be described as a sequence of recursive triggerings. To specify how an instance of the target schema $\mathrm{T}$ is produced by the chase, we define an injective function $\mu$ taking a term $f(\vec{b})$ with $f \in \mathrm{SK}, \vec{b} \in \mathrm{CONST}$, and returning a null $v \in$ NULLS. To save parentheses, we denote the null $\mu(f(\vec{b}))$ corresponding to the term $f(\vec{b})$, by $\hat{f}(\vec{b})$. Note that so encoded function $\mu$ applies to the whole term $f(\vec{b})$, even though, for the sake of readability, the hat symbol only appears over the function symbol $f$ and not over the arguments.

Definition 3.2 (Triggering). Let $I$ be an instance and $\sigma$ a nested tgd. A triggering $t$ of a part $\sigma_{i}$ of $\sigma$ (in its Skolemized form) with $\sigma_{i}=\forall \vec{x}\left(\varphi\left(\vec{x}_{0}, \vec{x}\right) \rightarrow \psi\left(\vec{x}_{0}, \vec{x}\right)\right)$, is a triple $\left(\sigma_{i}, \vec{a}_{0}, \vec{a}\right)$, where $\vec{a}$ is a tuple of constants assigned to the variables $\vec{x}$ and $\vec{a}_{0}$ is a tuple of constants assigned to the free variables $\vec{x}_{0}$ of $\sigma_{i}$ such that $I=\varphi\left(\vec{a}_{0}, \vec{a}\right)$ holds. The set facts $(t)$ consists of all facts in $\psi\left(\vec{a}_{0}, \vec{a}\right)$ obtained by substituting each instantiation of a Skolem term $f\left(\vec{a}^{\prime}\right)$ in $\psi\left(\vec{a}_{0}, \vec{a}\right)$ with $\hat{f}\left(\vec{a}^{\prime}\right)=\mu\left(f\left(\vec{a}^{\prime}\right)\right)$, where $\vec{a}^{\prime}$ is a prefix of the tuple $\vec{a}_{0} \cdot \vec{a}$. We define the set nulls $(t)$ to be $\emptyset$ if the tuple $\vec{x}$ is empty, otherwise, we set nulls $(t)=\left\{\hat{f}\left(\vec{a}_{0} \cdot \vec{a}\right) \mid f\left(\vec{a}_{0} \cdot \vec{a}\right)\right.$ occurs in $\left.\psi\left(\vec{a}_{0}, \vec{a}\right)\right\}$. That is, nulls $(t)$ is a set of nulls instantiating Skolem terms that occur in the part $\sigma_{i}$ but not in its ancestor parts in $\sigma$, that is, all Skolem terms in the set $\operatorname{vars}\left(\sigma_{i}\right)$.

Definition 3.3 (Chase with a nested $\operatorname{tg} d$, Chase forest). Let $I$ be an instance and $\sigma$ a nested tgd. Analogously to the tree structure of the parts of $\sigma$, we define the following tree structure of triggerings: Let $\sigma_{i}=\forall \vec{x}\left(\varphi_{i}\left(\vec{x}_{0}, \vec{x}\right) \rightarrow \psi_{i}\left(\vec{x}_{0}, \vec{x}\right)\right)$ be a part of $\sigma$ and let $t=\left(\sigma_{i}, \vec{a}_{0}, \vec{a}\right)$ be a triggering:

- if $\sigma_{i}$ is the root part of $\sigma$ (in which case $\vec{a}_{0}$ is empty), then $t$ is called a root triggering;

- if parent $\left(\sigma_{i}\right)=\sigma_{j}$ and there is a triggering $\left(\sigma_{j}, \vec{b}_{0}, \vec{b}\right)$ with $\vec{a}_{0}=\vec{b}_{0} \cdot \vec{b}$, then $\left(\sigma_{j}, \overrightarrow{b_{0}}, \vec{b}\right)$ is called the parent triggering of $t$.

The chase forest $\mathcal{F}_{\sigma, I}=\left(V_{\sigma, I}, T_{\sigma, I}\right)$ of $I$ with $\sigma$ is a rooted forest (i.e., a forest with a distinguished root for each connected component) whose set of vertices $V_{\sigma, I}$ is the set of all triggerings and where $T_{\sigma, I}$ contains an edge $\left(t^{\prime}, t\right)$ if $t^{\prime}$ is the parent triggering of $t$. For a set $\Sigma$ of nested tgds, the chase forest $\mathcal{F}_{\Sigma, I}$ of $I$ with $\Sigma$ is a pair $\left(V_{\Sigma, I}, T_{\Sigma, I}\right)$ with $V_{\Sigma, I}=\bigcup_{\sigma \in \Sigma} V_{\sigma, I}$ and $T_{\Sigma, I}=\bigcup_{\sigma \in \Sigma} T_{\sigma, I}$.

The chase of I with a nested $\operatorname{tg} d \sigma$, denoted as chase $(I, \sigma)$, is defined as the union of all facts of all triggerings in $\mathcal{F}_{\sigma, I}$, i.e., chase $(I, \sigma)=\bigcup_{t \in V_{\sigma, I}}$ facts $(t)$. Likewise, the chase of $I$ with a set $\Sigma$ of nested $\operatorname{tg} d s$, denoted as chase $(I, \Sigma)$, is defined as the union of all facts of all triggerings in $\mathcal{F}_{\Sigma, I}$, i.e., $\operatorname{chase}(I, \Sigma)=\bigcup_{t \in V_{\Sigma, I}} \operatorname{facts}(t)$.

Consider a triggering $t$ in $\mathcal{F}_{\Sigma, I}$. We refer to the subtree of $\mathcal{F}_{\Sigma, I}$ rooted at $t$ as the chase tree of $t$. The set of vertices in this subtree will be denoted as $\operatorname{desc}(t)$. Ancestor triggerings of $t$ are all vertices belonging to the path from the root to the parent of $t$ in $\mathcal{F}_{\Sigma, I}$. As follows from Definition 3.3, free variables $\vec{x}_{0}$ of the part associated with $t$ are bound in the ancestor triggerings of $t$ to a tuple of constants $\vec{a}_{0}$. We shall refer to $\vec{a}_{0}$ as the input assignment of $t$. If $t$ is a parent triggering of $t_{1}$, then we call $t_{1}$ a child triggering of $t$. If $t_{1}$ and $t_{2}$ are child triggerings of $t$, then we call $t_{1}$ and $t_{2}$ sibling triggerings. 


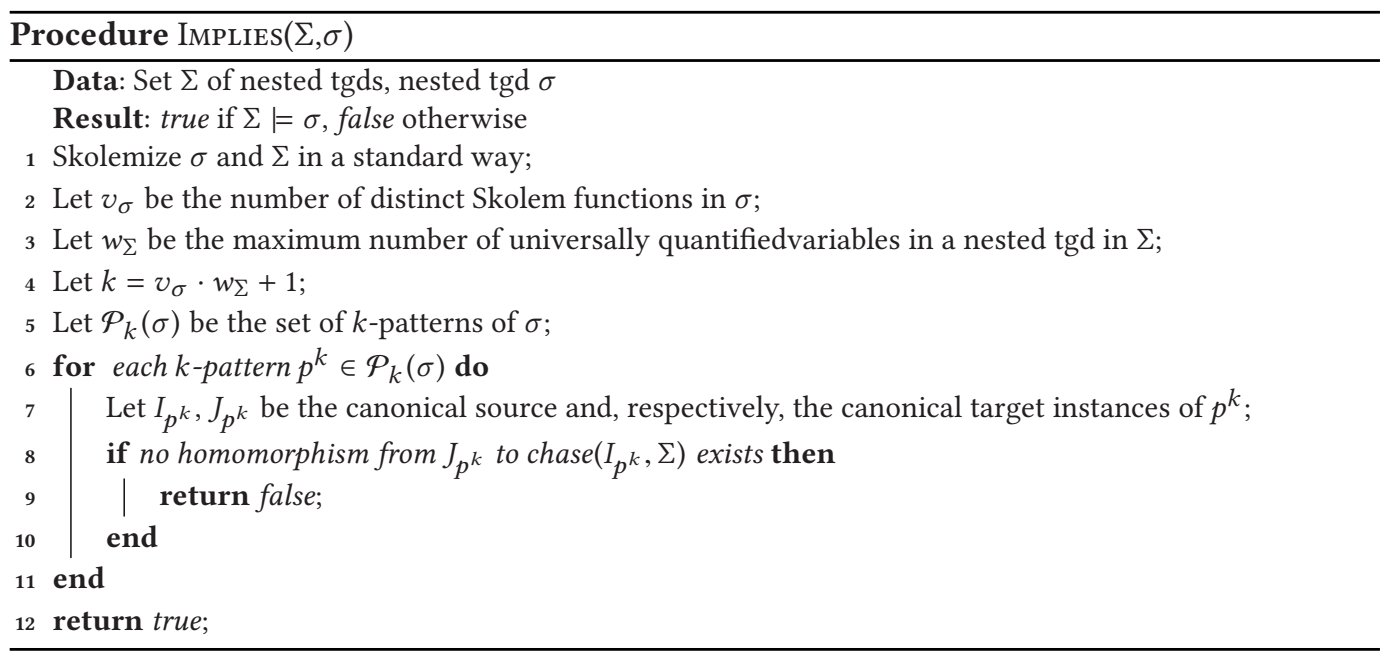

Proposition 3.4. Let I be a source instance and let $\Sigma$ be a set of nested tgds. Then, the instance chase $(I, \Sigma)$ is a universal solution for I under $\Sigma$. Namely, (i) $I \cup$ chase $(I, \Sigma) \mid=\Sigma$ and (ii) for each $J$ such that $(I, J) \mid=\Sigma$, we have chase $(I, \Sigma) \rightarrow J$.

PROOF. We refer to Reference [14] for a procedure converting a nested tgd into an equivalent SO tgd, essentially by Skolemizing it, and taking a conjunction of all parts of a nested tgd, where the antecedent of each nested part is extended with the antecedents of all its ancestors. From this, we can conclude that $\Sigma$ is logically equivalent to an SO $\operatorname{tgd} \sigma$. It is easy to verify that the chase of such an SO tgd [11] creates exactly the same instance (up to renaming of nulls) as the chase in Definition 3.3. Since the chase of $I$ with $\sigma$ results in a universal solution for $I$ under $\sigma$ [11], and $\sigma$ is logically equivalent to $\Sigma$, we have that $\operatorname{chase}(I, \Sigma)$ is a universal solution for $I$ under $\Sigma$.

The second property of the chase, underpinning the decision procedure, is that facts produced by triggerings in distinct chase trees share no nulls. A consequence of this observation is that reasoning about nested tgds may be restricted to source instances that give rise to a single chase tree. Finally, we will show that we only need to consider chase trees whose fan-out (i.e., the number of child nodes of any vertex) has an upper bound that depends on the mapping but not on any particular source instance.

Patterns, Canonical Instances of Patterns. The algorithm behind Theorem 3.1 is described in the decision procedure Implies. We now introduce the notions used in this procedure.

Definition 3.5 (Pattern). Let $\sigma$ be a nested tgd. A pattern of $\sigma$ is a vertex-labeled rooted tree whose labels are the parts $\sigma_{1}, \sigma_{2}, \ldots$ of $\sigma$, such that if $v$ is a child of $v^{\prime}$ in the pattern, then label $(v) \in$ $\operatorname{child}\left(\operatorname{label}\left(v^{\prime}\right)\right)$.

Let $\Sigma$ be a set of nested tgds, $\sigma \in \Sigma$, let $I$ be a source instance, and let $T$ be a tree in the chase forest $\mathcal{F}_{\Sigma, I}$ of $I$ with $\Sigma$. The pattern of $T$ is the vertex-labeled tree obtained from $T$ by relabeling each vertex with label $\left(\sigma_{i}, \vec{a}_{0}, \vec{a}\right)$ by $\sigma_{i}$.

Note that the pattern of any tree $T$ in a chase forest $\mathcal{F}_{\Sigma, I}$ is a pattern of some $\sigma \in \Sigma$. Conversely, not every pattern of a nested $\operatorname{tgd} \sigma$ is necessarily attainable as the pattern of some tree $T$ in the chase forest for some source instance $I$. For instance, consider the nested tgd $\sigma=\forall x_{1}\left(S_{1}\left(x_{1}\right) \rightarrow\left(\left(S_{2}\left(x_{1}\right) \rightarrow T\left(x_{1}\right)\right)\right)\right.$ with a single nested part. This tgd can only generate chase trees with patterns having at most two nodes. This is because the assignment of the only variable $x_{1}$ 


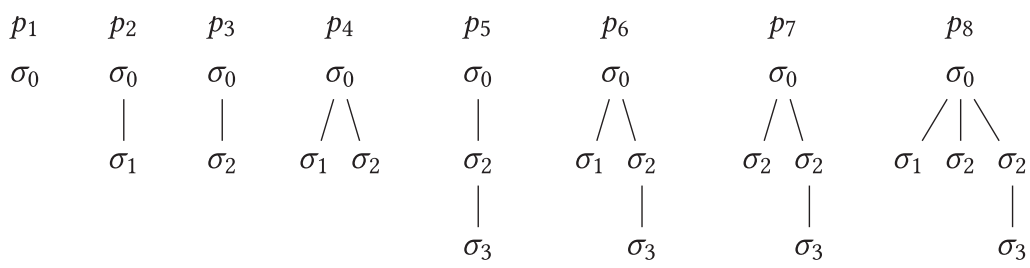

Fig. 2. 1-patterns of the $\operatorname{tgd} \sigma$ with the parts $\sigma_{0}, \ldots, \sigma_{3}$ from Example 2.2.

is determined by the root triggering, and thus only a single triggering of the nested part is possible. However, suppose that we modify $\sigma$ to $\sigma^{\prime}=\forall x_{1}\left(S_{1}\left(x_{1}\right) \rightarrow\left(\forall x_{2}\left(S_{2}\left(x_{2}\right) \rightarrow T\left(x_{1}, x_{2}\right)\right)\right)\right.$. Then, for appropriately chosen source instance $I$, the chase forest $\mathcal{F}_{\sigma^{\prime}, I}$ can give rise to patterns with an arbitrarily big number of nodes. For instance, for the source instance $I_{n}=\left\{S_{1}(a), S_{2}\left(b_{1}\right), \ldots, S_{2}\left(b_{n}\right)\right\}$, we get a pattern whose root node has $n$ child nodes.

We also use the notion of "subtree clones" in a pattern. Manipulating patterns by adding or removing clones will be a basic technique in many proofs of this article.

Definition 3.6 (Pattern subtree, clone, $k$-pattern). Let $\sigma$ be a nested tgd and let $p$ be a pattern of $\sigma$. By a subtree of a pattern $p$, we mean a complete subtree with all descendant nodes. We call $t^{\prime}$ a clone of $t$ if $t, t^{\prime}$ are isomorphic subtrees and their roots have the same parent node. Let $C_{t}$ denote the set containing $t$ and all its clones in $p$. If for each subtree $t,\left|C_{t}\right| \leq k$, we call $p$ a $k$-pattern. $\mathcal{P}_{k}(\sigma)$ is the set of all $k$-patterns of $\sigma$.

Example 3.7. Recall the nested tgd $\sigma$ with four parts from Example 2.2. The set $\mathcal{P}_{1}(\sigma)=$ $\left\{p_{1}, \ldots, p_{8}\right\}$ containing all 1-patterns of $\sigma$ is shown in Figure 2.

Proposition 3.8. For a positive integer $k$ and a nested $\operatorname{tg} d \sigma$, the set of $k$-patterns of $\sigma$ is finite.

Proof. Note that each part $\sigma_{i}$ of $\sigma$ can be obtained from some subformula $\forall \vec{x}(\varphi \rightarrow \psi)$ of $\sigma$ by omitting conjuncts with implications from $\psi$. Let $\lceil\cdot\rceil$ denote the function taking the part index $i$ and retrieving the subformula of $\sigma$ corresponding to the part $\sigma_{i}:\lceil i\rceil=\sigma_{i}\left(\vec{x}_{i}\right)$ where $\vec{x}_{i}$ denotes the free variables of the subformula. Syntactically, the sentence $\forall \vec{x}_{i} \sigma_{i}\left(\vec{x}_{i}\right)$ is a nested tgd. By slight abuse of notation, we use $\mathcal{P}_{k}(\lceil i\rceil)$ to denote the set of $k$-patterns of this nested tgd. Clearly, $\mathcal{P}_{k}(\lceil i\rceil)$ coincides with the set of all distinct subtrees in $\mathcal{P}_{k}(\sigma)$ with the root labeled by the part $\sigma_{i}$. We now proceed by structural induction on $\sigma$ to prove the proposition.

Base case: $\lceil i\rceil$ contains no nested implications. The set of $k$-patterns $\mathcal{P}_{k}(\lceil i\rceil)$ then consists of a single tree whose single node is labeled $\sigma_{i}$.

Now let $\lceil i\rceil=\forall \vec{x}\left(\varphi_{i} \rightarrow \psi_{i}\right)$ be such that $\psi_{i}$ is $\psi_{\alpha} \wedge\left\lceil i_{1}\right\rceil \wedge \ldots \wedge\left\lceil i_{n}\right\rceil$ where $\psi_{\alpha}$ is a conjunction of atoms and $\left\lceil i_{1}\right\rceil, \ldots,\left\lceil i_{n}\right\rceil$ are subformulas $\forall \vec{x}_{i_{j}}\left(\varphi_{i_{j}} \rightarrow \psi_{i_{j}}\right)$, for $j \in\{1 \ldots n\}$. The inductive hypothesis is that the sets $\mathcal{P}_{k}\left(\left\lceil i_{1}\right\rceil\right), \ldots, \mathcal{P}_{k}\left(\left\lceil i_{n}\right\rceil\right)$ of $k$-patterns are finite. The set $\mathcal{P}_{k}(\lceil i\rceil)$ contains all patterns that have (i) the root node labeled with $\sigma_{i}$, and (ii) up to $k$ copies of each pattern from the set $\bigcup_{j \in\{1 \ldots n\}} \mathcal{P}_{k}\left(\left\lceil i_{j}\right\rceil\right)$ as subtrees rooted at child nodes of the root. Since each set $\mathcal{P}_{k}\left(\left\lceil i_{j}\right\rceil\right)$ is finite, $\mathcal{P}_{k}(\lceil i\rceil)$ is finite as well. It follows that also the set $\mathcal{P}_{k}(\sigma)$ is finite (albeit non-elementary in $k)$.

Definition 3.9 (Canonical chase tree, canonical instances of a pattern). Let $p$ be a pattern of a nested tgd $\sigma$. The canonical chase tree $T_{p}$ of $p$ is a labeled tree isomorphic to $p$ : for every node labeled $\sigma_{i}$ in $p$, there is a unique triggering $t=\left(\sigma_{i}, \vec{a}_{0}, \vec{a}\right)$ in $T_{p}$, such that $\vec{a}$ assigns fresh, pairwisedistinct constants to the universally quantified variables of $\sigma_{i}$, and $\vec{a}_{0}$ is either empty if $t$ is a root triggering, or equal to $\vec{b}_{0} \cdot \vec{b}$ if $\left(\sigma_{j}, \vec{b}_{0}, \vec{b}\right)$ is the parent triggering of $t$. The canonical source instance $I_{p}$, respectively, canonical target instance $J_{p}$, of the chase tree $T_{p}$ are defined as minimal instances 


$$
\left(\sigma_{1},\left(a_{1}\right),\left(a_{2}\right)\right): \frac{S_{2}\left(a_{2}\right)}{R_{2}\left(\hat{f}\left(a_{1}\right), a_{2}\right)}\left(\sigma_{0},(),\left(a_{1}\right)\right): \frac{S_{1}\left(a_{1}\right)}{\emptyset}
$$

Fig. 3. Canonical chase tree of the pattern $p_{8}$ and the facts of its canonical source instance $I_{p_{8}}$ (above the bars) and the canonical target instance $J_{p_{8}}$ (below the bars).

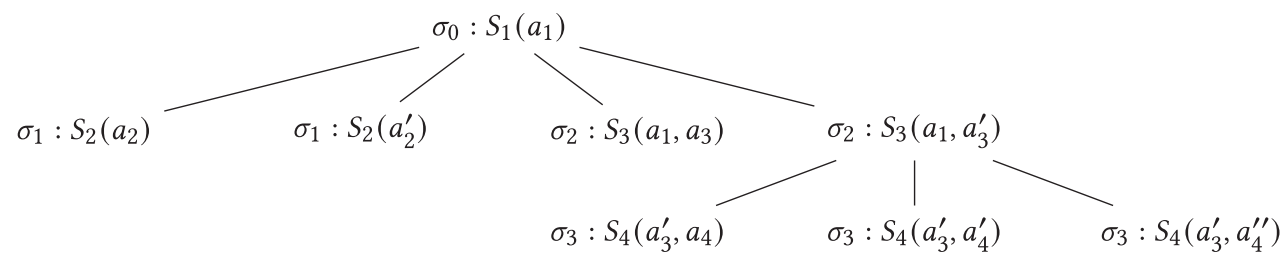

Fig. 4. A 3-pattern and the facts constituting its canonical source instance.

such that for each node $t=\left(\sigma_{i}, \vec{a}_{0}, \vec{a}\right)$ of $T_{p}$ with $\sigma_{i}=\forall \vec{x}\left(\varphi\left(\vec{x}_{0}, \vec{x}\right) \rightarrow \psi\left(\vec{x}_{0}, \vec{x}\right)\right)$, the atoms of $\varphi\left(\vec{a}_{0}, \vec{a}\right)$ are contained in $I_{p}$ and the atoms of $\psi\left(\vec{a}_{0}, \vec{a}\right)$-substituting every instantiation $f\left(\vec{a}^{\prime}\right)$ of a Skolem term in $\psi\left(\vec{a}_{0}, \vec{a}\right)$ by a fresh null $\hat{f}\left(\vec{a}^{\prime}\right)$-are in $J_{p}$, i.e., facts $(t) \subseteq J_{p}$ holds.

Note that the canonical target instance $J_{p}$ is, in general, not a solution of the canonical source instance $I_{p}$, since it may not be fully chased. Moreover, note that we often speak of the canonical source and target instances of a pattern $p$ instead of $T_{p}$, even though the constants used to create $T_{p}$ from $p$ can be arbitrary. The justification is that such instances are unique up to renaming of constants, and the concrete names of the constants are irrelevant for our further considerations.

Example 3.10. Figure 3 shows the canonical chase tree $T_{p_{8}}$, canonical source instance $I_{p_{8}}$, and the canonical target instance $J_{p_{8}}$ of the 1-pattern $p_{8}$ from Example 3.7 .

The next example shows the canonical source instance of a pattern containing clones of subtrees.

Example 3.11. One possible 3-pattern based on the 1-pattern $p_{8}$ from Example 3.10, in which one clone of the node $\sigma_{1}$ and two clones of the node $\sigma_{3}$ are added, is shown in Figure 4, along with the facts of its canonical source instance.

Finally, we have the stage set to put the procedure IMPLIES into action.

Example 3.12. Consider the nested $\operatorname{tgd} \tau$ and the s-t $\operatorname{tgds} \tau^{\prime}$ and $\tau^{\prime \prime}$ :

$$
\begin{aligned}
& \tau: \forall x_{1}\left(S_{1}\left(x_{1}\right) \rightarrow \exists y\left(\forall x_{2} S_{2}\left(x_{2}\right) \rightarrow R\left(x_{2}, y\right)\right)\right), \\
& \tau^{\prime}: \forall x_{1} \forall x_{2}\left(S_{1}\left(x_{1}\right) \wedge S_{2}\left(x_{2}\right) \rightarrow \exists z R\left(x_{2}, z\right)\right), \\
& \tau^{\prime \prime}: \forall x_{1} \forall x_{2}\left(S_{1}\left(x_{1}\right) \wedge S_{2}\left(x_{2}\right) \rightarrow R\left(x_{2}, x_{1}\right)\right) .
\end{aligned}
$$

We now use the procedure Implies to test if any of $\tau^{\prime}, \tau^{\prime \prime}$ implies $\tau$. The first step is the Skolemization of $\tau$ :

$$
\begin{array}{lrl}
\tau_{1}: & \forall x_{1}\left(S_{1}\left(x_{1}\right) \rightarrow\right. \\
\tau_{2}: & \left.\forall x_{2}\left(S_{2}\left(x_{2}\right) \rightarrow R\left(x_{2}, f\left(x_{1}\right)\right)\right)\right) .
\end{array}
$$




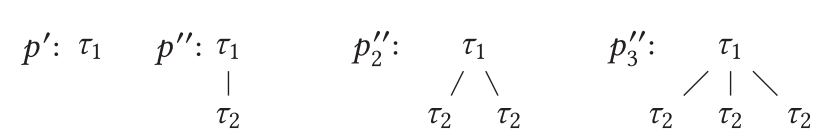

Fig. 5. Patterns used to test $\tau^{\prime}=\tau$ and $\tau^{\prime \prime} \mid=\tau$.

According to line 4 of the procedure, the bound $k$ on the number of clones should be 3 for testing $\tau^{\prime} \mid=\tau$ and $\tau^{\prime \prime} \mid=\tau$, since we have $v_{\tau}=1$ and $w_{\left\{\tau^{\prime}\right\}}=w_{\left\{\tau^{\prime \prime}\right\}}=2$. The set $\mathcal{P}_{3}(\tau)$ has two 1-patterns $\left\{p^{\prime}, p^{\prime \prime}\right\}$, of which only $p^{\prime \prime}$ has a non-empty canonical target instance. Based on $p^{\prime \prime}$, the 2-pattern $p_{2}^{\prime \prime}$ and the 3-pattern $p_{3}^{\prime \prime}$ can be obtained. One can then check that the set $\left\{p^{\prime}, p^{\prime \prime}, p_{2}^{\prime \prime}, p_{3}^{\prime \prime}\right\}$ in Figure 5 is actually the complete set of 3-patterns of $\tau$.

Let $I_{p}$ and $J_{p}$ denote the canonical source, respectively, canonical target instance, of a pattern $p$ of $\tau$ with $p \in\left\{p^{\prime}, p^{\prime \prime}, p_{2}^{\prime \prime}, p_{3}^{\prime \prime}\right\}$. Let $\Sigma$ be one of $\left\{\tau^{\prime}\right\}$ or $\left\{\tau^{\prime \prime}\right\}$. To test if $\Sigma=\tau$ holds, the procedure IMPLIES checks the existence of a homomorphism from $J_{p}$ to $\operatorname{chase}\left(I_{p}, \Sigma\right)$ for all patterns $p \in\left\{p^{\prime}, p^{\prime \prime}\right.$, $\left.p_{2}^{\prime \prime}, p_{3}^{\prime \prime}\right\}$ (for the case $\Sigma=\left\{\tau^{\prime \prime}\right\}$ ). For $p^{\prime}$, this check is trivial, since $p^{\prime}$ has an empty canonical target instance. We illustrate the check for the pattern $p_{2}^{\prime \prime}$. The canonical source and target instances for this pattern are as follows:

$$
I_{p_{2}^{\prime \prime}}=\left\{S_{1}\left(a_{1}\right), S_{2}\left(a_{2}\right), S_{2}\left(a_{2}^{\prime}\right)\right\}, J_{p_{2}^{\prime \prime}}=\left\{R_{2}\left(a_{2}, \hat{f}\left(a_{1}\right)\right), R_{2}\left(a_{2}^{\prime}, \hat{f}\left(a_{1}\right)\right)\right\} .
$$

The Skolemization of $\tau^{\prime}$ yields $\forall x_{1} \forall x_{2}\left(S_{1}\left(x_{1}\right) \wedge S_{2}\left(x_{2}\right) \rightarrow R\left(x_{2}, g\left(x_{1}, x_{2}\right)\right)\right)$ and the chase of $I_{p_{2}^{\prime \prime}}$ with $\tau^{\prime}$ results in $J_{\tau^{\prime}}=\left\{R\left(a_{2}, g\left(a_{1}, a_{2}\right)\right), R\left(a_{2}^{\prime}, g\left(a_{1}, a_{2}^{\prime}\right)\right)\right\}$. There is no homomorphism from $J_{p_{2}^{\prime \prime}}$ to $J_{\tau^{\prime}}$ and thus ImPLies $\left(\left\{\tau^{\prime}\right\}, \tau\right)$ outputs false, indicating $\tau^{\prime} \mid \vDash \tau$. The failure of implication is because the existentially quantified variable in $\tau^{\prime}$ depends on both $x_{1}$ and $x_{2}$, but only on $x_{1}$ in $\tau$.

We now check if $\tau^{\prime \prime}=\tau$ holds. The chase of $I_{p_{2}^{\prime \prime}}$ with $\tau^{\prime \prime}$ results in $J_{\tau^{\prime \prime}}=\left\{R\left(a_{2}, a_{1}\right), R\left(a_{2}^{\prime}, a_{1}\right)\right\}$. The mapping $\left[\hat{f}\left(a_{1}\right) \mapsto a_{1}\right]$ is a homomorphism from $J_{p_{2}^{\prime \prime}}$ to $J_{\tau^{\prime \prime}}$, and thus the test at line 8 of the procedure IMPLIES passes successfully for the pattern $p_{2}^{\prime \prime}$. One can verify that so do the checks for the patterns $p^{\prime \prime}$ and $p_{3}^{\prime \prime}$. Therefore, Implies $\left(\left\{\tau^{\prime \prime}\right\}, \tau\right)$ outputs true, indicating $\tau^{\prime \prime} \mid=\tau$. Indeed, where $\tau^{\prime \prime}$ yields the value of $x_{1}, \tau$ only requires some value (that is, a null) determined by the assignment of $x_{1}$, which is a strictly weaker condition.

\subsection{Proof of Theorem 3.1}

Two ideas underlie the correctness of the procedure ImpLIEs. The first is a well-known property of schema mappings that are closed under target homomorphisms and allow for universal solutions for every source instance. Namely, $\Sigma \mid=\sigma$ if and only if for every source instance $I$, there is a homomorphism from a universal solution $J_{I}^{\sigma}$ for $I$ under $\sigma$ into all solutions for $I$ under $\Sigma$. By Proposition 3.4 it suffices to homomorphically embed $\operatorname{chase}(I, \sigma)$ into $\operatorname{chase}(I, \Sigma)$, which is equivalent to embedding every f-block of $\operatorname{chase}(I, \sigma)$ in $\operatorname{chase}(I, \Sigma)$ independently [10]. This is not a feasible test, however, since enumerating all source instances is required. Our main finding in this section is how to address this issue for nested tgds: We show that for arbitrary $I$, chase $(I, \sigma) \rightarrow \operatorname{chase}(I, \Sigma)$ holds if for every $k$-pattern $p^{k} \in \mathcal{P}_{k}(\sigma)$, a homomorphism from $J_{p^{k}}$ to $\operatorname{chase}\left(I_{p^{k}}, \Sigma\right)$ exists, where $k$ is a constant depending on $\sigma$ and $\Sigma$, as defined at line 4 of the procedure IMPLIES, and $I_{p^{k}}$ and $J_{p^{k}}$ are the canonical source instance and, respectively, the canonical target instance of $p^{k}$. That is, instead of an infinite number of source instances, one can enumerate a finite set of patterns.

The proof of this latter idea makes use of special notation and basic facts about chase trees, which we present next.

Active domain of the chase result. The chase with nested tgds identifies nulls with instantiations of Skolem terms. By Definition 3.3, for every fact $R\left(e_{1}, \ldots, e_{m}\right)$ in $\operatorname{chase}(I, \Sigma)$ and for every $i \in\{1, \ldots, m\}, e_{i}$ is either a constant from $\operatorname{dom}(I)$ or a null $\hat{f}(\vec{a})=\mu(f(\vec{a}))$, where $f(\vec{x})$ is a Skolem 
function in $\Sigma, \vec{a}$ is a tuple of constants from $\operatorname{dom}(I)$ instantiating the variables $\vec{x}$ during the chase, and $\mu$ is an injective function mapping the Skolem term $f(\vec{a})$ onto the null $\hat{f}(\vec{a}) \in$ NULLS. By the injectivity of $\mu$, we can define an inverse function $\mu^{-1}$ from NULLS to the set of Skolem terms SK $\times$ CONST ${ }^{*}$. This inverse function allows us to formally define an operation of extracting all nulls used to build a chase instance.

Definition 3.13. Let $J$ be a target instance produced by the chase with a nested tgd. We define the set $\operatorname{cst}(J)$ as the set containing $\operatorname{dom}(J) \cap$ CONST plus all constants occurring within terms $\mu^{-1}(e)$ for every $e \in \operatorname{dom}(J) \cap$ NULLS.

The relationship between the sets $\operatorname{dom}(J)$ and $\operatorname{cst}(J)$ is thus the following: If $J$ consists solely of constants (e.g., $J$ is a source instance), then we have $\operatorname{dom}(J)=\operatorname{cst}(J)$. Otherwise, $\operatorname{dom}(J) \backslash \operatorname{cst}(J)$ is the set of nulls of $J$ and $\operatorname{cst}(J) \backslash \operatorname{dom}(J)$ is the set of constants that only occur in the tuple $\vec{a}$ of arguments of a term $f(\vec{a})$ associated by $\mu$ with some null in $\operatorname{dom}(J)$. As specified above, we use $\hat{f}(\vec{a})$ as a shorthand for $\mu(f(\vec{a}))$.

Independence of chase trees. Note that by the definition of the chase, nulls created by the chase with nested tgds correspond to Skolem terms of a specific structure: If a null $\hat{f}(\vec{a})$ is introduced by a non-root triggering $t$ for the first time in the chase, then all the constants used in any of the ancestor triggerings of $t$ occur in a prefix of $\vec{a}$. This observation provides a means of restricting the set of nulls that connect chase fragments generated by different triggerings.

Lemma 3.14. Let $\Sigma$ be a set of nested tgds, let I be a source instance for $\Sigma$, and let $J=$ chase $(I, \Sigma)$. Consider a triggering $\left(\sigma_{i}, \vec{a}_{0}, \vec{a}\right)$ in the chase forest of $I$ with $\Sigma$ and a subinstance $J^{\prime}$ of $J$ defined as follows: $J^{\prime}=\bigcup\left\{\right.$ facts $\left.(t) \mid t \in \operatorname{desc}\left(\left(\sigma_{i}, \vec{a}_{0}, \vec{a}\right)\right)\right\}$. If a null $\hat{f}(\vec{b})$ occurs both in $J^{\prime}$ and in $J \backslash J^{\prime}$, then (i) the variables $\vec{x}$ in the Skolem term $f(\vec{x})$, which $f(\vec{b})$ instantiates, appear free in $\sigma_{i}$ and (ii) $\vec{b}$ is a prefix of $\vec{a}_{0}$.

Proof. We first prove by contradiction that $\vec{b}$ is a prefix of $\vec{a}_{0}$. Suppose that $\vec{b}$ is not a prefix of $\vec{a}_{0}$. By the definition of triggering and the convention that variables in Skolem terms appear in the order they are quantified in the nested tgd, each null in $J^{\prime}$ has the form $g(\vec{c})$ such that either $\vec{c}=\vec{a}_{0} \cdot \vec{a}$ or the shorter tuple in the pair $\left(\vec{c}, \vec{a}_{0} \cdot \vec{a}\right)$ is a proper prefix of the longer one. Therefore, it must be the case that $\vec{a}_{0}$ is a proper prefix of $\vec{b}$. Since $J^{\prime}$ consists of facts generated by triggerings in $\operatorname{desc}\left(\left(\sigma_{i}, \vec{a}_{0}, \vec{a}\right)\right)$, we have that $\vec{b}$ must be prefixed by $\vec{a}_{0} \cdot \vec{a}$. From this and the assumption $\hat{f}(\vec{b}) \in \operatorname{dom}\left(J^{\prime}\right)$, we have that the Skolem function $f$ replaces a variable existentially quantified in $\operatorname{desc}\left(\sigma_{i}\right)$. Let $t^{\prime}=\left(\sigma_{j}, \vec{a}_{0}^{\prime}, \vec{a}^{\prime}\right)$ be an arbitrary triggering such that $\hat{f}(\vec{b})$ occurs in facts $\left(t^{\prime}\right)$ and $t^{\prime} \notin \operatorname{desc}(t)$. The existence of $t^{\prime}$ follows from the assumption of the lemma that $\hat{f}(\vec{b})$ occurs in the subinstance $\operatorname{chase}(I, \Sigma) \backslash \bigcup\left\{\operatorname{facts}(t) \mid t \in \operatorname{desc}\left(\left(\sigma_{i}, \vec{a}_{0}, \vec{a}\right)\right)\right\}$. Since $\sigma_{j} \in \operatorname{desc}\left(\sigma_{i}\right)$ and $\vec{b}$ is prefixed by $\vec{a}_{0} \cdot \vec{a}$, we have that $t^{\prime}$ must be contained in $\operatorname{desc}(t)$, which is a contradiction. We have shown that $\vec{b}$ must be a prefix of $\vec{a}_{0}$, and since $\vec{a}_{0}$ is the input assignment of $\left(\sigma_{i}, \vec{a}_{0}, \vec{a}\right), \vec{b}$ instantiates exclusively the variables that occur free in $\sigma_{i}$.

The following corollary of Lemma 3.14 is crucial for the correctness of the procedure IMPLIES:

Corollary 3.15. Consider a finite set $\Sigma$ of nested $t g d s$, a source instance $I$, and subinstances $J_{1}, J_{2}$ of chase $(I, \Sigma)$ produced by distinct chase trees in the chase forest of I with $\Sigma$. That is, $J_{1}=\bigcup\{$ facts $(t) \mid$ $\left.t \in \operatorname{desc}\left(t_{1}\right)\right\}, J_{2}=\bigcup\left\{f a c t s(t) \mid t \in \operatorname{desc}\left(t_{2}\right)\right\}$ for distinct root triggerings $t_{1}, t_{2}$. Then, $J_{1}$ and $J_{2}$ have no nulls in common. 
Proof. By Lemma 3.14, a null $\hat{f}(\vec{b})$ occurring both in $J_{1}$ and in $J_{2}$ must be such that $\vec{b}$ is a prefix of the input assignment of both $t_{1}$ and $t_{2}$, and thus (since $t_{1}$ and $t_{2}$ are root triggerings) $\vec{b}$ must be the empty tuple. However, the arguments of a Skolem term have to be non-empty.

Transforming patterns by increments. We now turn to $k$-patterns as the main tool of the procedure ImPLIEs. The following definition facilitates the reasoning about patterns obtained by inserting new clones of subtrees.

Definition $3.16\left(k^{+}\right.$-increment of a pattern). Let $p$ be a pattern of a nested $\operatorname{tg} d \sigma$. An increment of $p$ is obtained by (i) choosing a node $v$ of $p$, (ii) choosing a subtree $\pi$ rooted at some child node of $v$, and (iii) adding to $p$ a clone $\pi^{\prime}$ of $\pi$.

An increment is called a $k^{+}$-increment of $p$ if, in the above definition of an increment, the newly inserted subtree $\pi^{\prime}$ has at least $k$ clones in $p$ (and thus becomes one of at least $k+1$ isomorphic subtrees rooted at $v$ ).

We write $p \leq p^{\prime}$ if pattern $p^{\prime}$ can be obtained from a pattern $p$ by a sequence of zero or more increments, and we write $p \leq_{k} p^{\prime}$ if $p^{\prime}$ can be obtained from $p$ by a sequence of zero or more $k^{+}$-increments.

Equipped with this notation, we formulate a property of $k$-patterns that underpins the proof of Theorem 3.1. It will also be used in the proof of Theorem 4.14 in Section 4 on the structure of the core of nested tgds.

Lemma 3.17. For every positive integer $k$ and for every pattern $p$ of $\sigma$, there is a $k$-pattern $p^{k} \in$ $\mathcal{P}_{k}(\sigma)$ such that $p^{k} \leq_{k} p$ holds. Moreover, $p$ can be obtained from $p^{k}$ by applying $k^{+}$-increment steps in such a way that whenever a subtree $t$ is added, then for every subtree $t^{\prime}$ of $t$, the set $C_{t^{\prime}}$ of all clones of $t^{\prime}$ (according to Definition 3.6) satisfies the condition $\left|C_{t^{\prime}}\right| \leq k$.

Intuitively, the above condition $\left|C_{t^{\prime}}\right| \leq k$ for subtrees $t^{\prime}$ of cloned subtrees $t$ means that the $k^{+}$-increment steps are applied in a top-down fashion, i.e., it cannot happen that some subtree $t^{\prime}$ is cloned and later a subtree $t$ containing $t^{\prime}$ is also cloned. This fact will be important in Theorem 4.14 to get an appropriate upper bound on the size of any cloned subtree $t$.

Proof. We consider the inverse operation of $k^{+}$-increments, namely, $k^{+}$-elimination defined as follows: Let $v$ be a node in a pattern $p$ such that there are $k^{\prime}>k$ isomorphic subtrees rooted at the child nodes of $v$. Then, we may delete $k^{\prime}-k$ of these subtrees.

Below, we will show that there exists a pattern $p^{k} \in \mathcal{P}_{k}(\sigma)$ that can be obtained from $p$ by a sequence of $k^{+}$-elimination steps in such a way that every subtree $t^{\prime}$ of an eliminated subtree $t$ satisfies the condition $\left|C_{t^{\prime}}\right| \leq k$. The desired transformation of $p^{k}$ into $p$ is then obtained by applying the sequence of corresponding $k^{+}$-increment steps in inverse order.

The proof is by induction on the number $n$ of " $k$-violations" in pattern $p$, i.e., the number of pairs $\left(v, C_{t}\right)$ where $v$ is a node in $p$ and $C_{t}$ is a set of clones rooted at child nodes of $v$ such that $\left|C_{t}\right|>k$.

For the induction begin, if $n=0$, then $p \in \mathcal{P}_{k}(\sigma)$ and we are done. For the induction step, suppose that $p$ has $n>0 k$-violations. We choose a node $v$ at maximal depth in $p$, such that there are $k^{\prime}>k$ isomorphic subtrees rooted at the child nodes of $v$. By our assumption of maximal depth, we conclude that every subtree $t^{\prime}$ of any of these isomorphic subtrees satisfies the condition $\left|C_{t^{\prime}}\right| \leq k$. We can transform $p$ into $p^{\prime}$ by a $k^{+}$-elimination step that deletes $k^{\prime}-k$ of these isomorphic subtrees. The resulting pattern $p^{\prime}$ has only $n-1 k$-violations. Hence, by the induction hypothesis, $p^{\prime}$ can be transformed into a pattern $p^{k} \in \mathcal{P}_{k}(\sigma)$ via a sequence of $k^{+}$-elimination steps such that every subtree $t^{\prime}$ of an eliminated subtree $t$ satisfies the condition $\left|C_{t^{\prime}}\right| \leq k$. Hence, by first transforming $p$ into $p^{\prime}$ and then $p^{\prime}$ into $p^{k}$, we get the desired transformation from $p$ into $p^{k}$. 
Abstracting chase trees via canonical target instances. It turns out that, to test the condition $\operatorname{chase}(I, \sigma) \rightarrow \operatorname{chase}(I, \Sigma)$, it suffices to use canonical instances of patterns realized in the chase tree of $I$ with $\sigma$. This way one can, e.g., abstract away from undesired multiple occurrences of constants in the facts of $I$.

Lemma 3.18. Let $\sigma$ be a nested $\operatorname{tgd}$, let $\Sigma$ be a set of nested $\operatorname{tgds}$, and let I be an arbitrary source instance. Moreover, let $\mathcal{P}$ be the set of patterns of the chase forest of I with $\sigma$. Then, if for each pattern $p$ in $\mathcal{P}, J_{p} \rightarrow \operatorname{chase}\left(I_{p}, \Sigma\right)$ holds, where $I_{p}, J_{p}$ are the canonical sources, respectively, target instances, of $p$, then chase $(I, \sigma) \rightarrow \operatorname{chase}(I, \Sigma)$ holds as well.

Note that the opposite direction of the above lemma does, in general, not hold, i.e.: It is not guaranteed that chase $(I, \sigma) \rightarrow \operatorname{chase}(I, \Sigma)$ implies $J_{p} \rightarrow \operatorname{chase}\left(I_{p}, \Sigma\right)$. However, this is irrelevant, since the only purpose of Lemma 3.18 is to restrict the set of source instances that have to be considered in the implication test of nested tgds: In principle, we would have to test that $\operatorname{chase}(I, \sigma) \rightarrow \operatorname{chase}(I, \Sigma)$ holds for every possible source instance. Lemma 3.18 tells us that we may restrict ourselves to canonical source instances. That is, we get rid of undesired double occurrences of constants. Below, in Lemma 3.22, we will prove a further restriction of the source instances that have to be considered. More precisely, we will show in Lemma 3.22 that we may restrict ourselves to canonical source instances up to a certain size. The decidability of the implication problem of nested tgds (see the proof of Theorem 3.1 at the end of this section) will then be an easy consequence.

The proof of Lemma 3.18 uses a notion of homomorphism that affects the constants of an instance and preserves function symbols in nulls, in contrast to the usual constant-preserving homomorphism acting on nulls, as defined in Section 2.

Definition 3.19 (C-mapping, c-homomorphism). A c-mapping is a function $\theta:$ CONST $\rightarrow$ CONST that is naturally extended to Skolem terms with arguments from CONST: For every $f\left(a_{1}, \ldots, a_{k}\right)$, we define $\theta\left(f\left(a_{1}, \ldots, a_{k}\right)\right)=f\left(\theta\left(a_{1}\right), \ldots, \theta\left(a_{k}\right)\right)$. For $e \in$ NULLS, we then let $\theta(e)=\mu\left(\theta\left(\mu^{-1}(e)\right)\right)$ or, using the shorthand notation, $\theta(\hat{f}(\vec{a}))=\hat{f}(\theta(\vec{a}))$. The latter extension leads to a function $\theta^{\text {[null] }}$ : NULLS $\rightarrow$ NULLS derived from the c-mapping $\theta$. For tuples of constants and nulls, we define $\theta\left(\left(s_{1}, \ldots, s_{k}\right)\right)=\left(\theta\left(s_{1}\right), \ldots, \theta\left(s_{k}\right)\right)$. Finally, a c-mapping $\theta$ is called a $c$-homomorphism from $J$ to $J^{\prime}$ if for every fact $R(\vec{s}) \in J$, there is a fact $R(\theta(\vec{s})) \in J^{\prime}$.

Example 3.20. There is a c-homomorphism $\theta=\left[a \mapsto a^{\prime}, b \mapsto b^{\prime}\right]$ transforming the instance $J=$ $\{R(a, b, \hat{f}(a, b))\}$ into $J^{\prime}=\left\{R\left(a^{\prime}, b^{\prime}, \hat{f}\left(a^{\prime}, b^{\prime}\right)\right)\right\}$.

Proof of Lemma 3.18. By Corollary 3.15, subinstances of chase $(I, \sigma)$ generated by distinct chase trees have no nulls in common. Therefore, a homomorphism from chase $(I, \sigma)$ to chase $(I, \Sigma)$ exists if and only if for every chase tree $T$ in $\mathcal{F}_{\sigma, I}$, the condition $J \rightarrow \operatorname{chase}(I, \Sigma)$ holds, where $J=\bigcup\{f a c t s(t) \mid t$ is a node of $T\}$.

Fix an arbitrary chase tree $T$ in $\mathcal{F}_{\sigma, I}$ with $t_{0}$ denoting its root triggering, and let $J=\bigcup\{$ facts $(t) \mid$ $t$ be a node of $T$ \}. We use $p$ to denote the pattern of $T$, and we denote by $T_{p}$ the canonical chase tree of $p$, i.e., $T_{p}$ is a chase tree where the triggerings assign fresh, pairwise-distinct constants to variables. Furthermore, let $I_{p}$ be the canonical source instance and let $J_{p}$ be the canonical target instance. Since the chase tree $T$ is arbitrary, it is sufficient to show that $J_{p} \rightarrow \operatorname{chase}\left(I_{p}, \Sigma\right)$ implies $J \rightarrow \operatorname{chase}(I, \Sigma)$. Under the assumption that a homomorphism $h$ from $J_{p}$ to $\operatorname{chase}\left(I_{p}, \Sigma\right)$ exists, we will construct a homomorphism $e$ from $J$ to $\operatorname{chase}(I, \Sigma)$.

Since $p$ is a pattern of $T$, there is a one-to-one correspondence $\theta^{[\mathrm{tr}]}$ between the triggerings in $T$ and those in the canonical chase tree $T_{p}$. Since triggerings in $T_{p}$ assign fresh, pairwise-distinct 


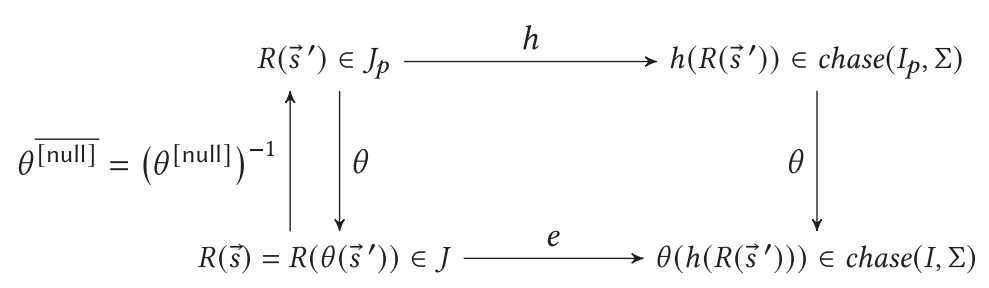

Fig. 6. Mappings used in the proof of Lemma 3.18.

constants to variables, there is a c-homomorphism $\theta$ from $I_{p}$ to $I$, which, by extending its domain to nulls according to Definition 3.19 is also a c-homomorphism from $J_{p}$ to $J$. Specifically, for every triggering $\left(\sigma_{i}, \vec{a}_{0}, \vec{a}\right)$ in $T$ and triggering $\left(\sigma_{i}, \vec{a}_{0}^{\prime}, \vec{a}^{\prime}\right)$ in $T_{p}$ such that $\theta^{[\operatorname{tr}]}\left(\left(\sigma_{i}, \vec{a}_{0}^{\prime}, \vec{a}^{\prime}\right)\right)=\left(\sigma_{i}, \vec{a}_{0}, \vec{a}\right)$, $\theta$ maps the elements of $\vec{a}^{\prime}$ to the respective elements of $\vec{a}$. Consider the mapping $\theta^{\text {[null] }}$ on nulls of $J_{p}$, derived from $\theta$ as in Definition 3.19. In Definition 3.2, the function nulls is defined, which, for a triggering $\left(\sigma_{j}, \vec{b}_{0}, \vec{b}\right)$, retrieves all nulls $\hat{f}_{i}\left(\vec{b}_{0} \cdot \vec{b}\right)$ instantiating Skolem terms from $\operatorname{vars}\left(\sigma_{j}\right)$. Note that $\bigcup\left\{\right.$ nulls $(t) \mid t$ a node of $\left.T_{p}\right\}$ is exactly the set $N_{p}$ of nulls of $J_{p}: N_{p}=\operatorname{dom}\left(J_{p}\right) \cap$ NULLS. Similarly, the set $N$ of nulls of $J$ is $\bigcup\{$ nulls $(t) \mid t$ a node of $T\}$.

We claim that $\theta^{[\text {null] }}$ is a bijection between the nulls $N_{p}$ in $J_{p}$ and the nulls $N$ in $J$. This can be seen as follows: By Corollary 3.15, we know that any two different triggerings introduce disjoint sets of nulls. Moreover, $\theta^{[\mathrm{tr}]}$ is a bijection between triggerings in $T_{p}$ and in $T$. Hence, it suffices to show that, for every single triggering $t$ in $T_{p}$, the mapping $\theta^{[\text {null] }}$ is a bijection from nulls $(t)$ to nulls $\left(\theta^{[\operatorname{tr}]}(t)\right)$. Let $\left.t=\left(\sigma_{i}, \vec{a}_{0}^{\prime}, \vec{a}^{\prime}\right)\right)$ and $\theta^{[\operatorname{tr}]}(t)=\left(\sigma_{i}, \vec{a}_{0}, \vec{a}\right)$. Moreover, let $\vec{x}_{0}$ denote the universally quantified variables of all ancestors of part $\sigma_{i}$ and let $\vec{x}$ denote the universally quantified variables of part $\sigma_{i}$. Then the Skolem terms introducing nulls by a triggering of $\sigma_{i}$ are all of the form $f_{1}\left(\vec{x}_{0}, \vec{x}\right), \ldots, f_{m}\left(\vec{x}_{0}, \vec{x}\right)$ for some $m \geq 0$. We thus have nulls $(t)=\left\{f_{1}\left(\vec{a}_{0}^{\prime}, \vec{a}^{\prime}\right), \ldots, f_{m}\left(\vec{a}_{0}^{\prime}, \vec{a}^{\prime}\right)\right\}$ and nulls $\left(\theta^{[\operatorname{tr}]}(t)\right)=\left\{f_{1}\left(\vec{a}_{0}, \vec{a}\right), \ldots, f_{m}\left(\vec{a}_{0}, \vec{a}\right)\right\}$. In other words, the triggering of such a part generates $m$ distinct nulls (corresponding to the $m$ existentially quantified variables in the non-Skolemized form) no matter if in this triggering some of the variables in $\left(\vec{x}_{0}, \vec{x}\right)$ are assigned the same constant. Hence, $\theta^{[\mathrm{null}]}$ is indeed a bijection from nulls $(t)$ to $\operatorname{nulls}\left(\theta^{[\mathrm{tr}]}(t)\right)$.

This observation is crucial for our construction, which will be using $\theta^{\overline{[\text { null] }}}$, the inverse of $\theta^{\text {[null] }}$, that is $\theta^{\overline{\text { null] }}}=\left(\theta^{\text {[null] }}\right)^{-1}: N \rightarrow N_{p}$. Specifically, our desired homomorphism $e$ from $J$ to $\operatorname{chase}(I, \Sigma)$ will be defined as the composition of $\theta^{\overline{\text { [null] }}}$ with $h$ and $\theta^{\text {[null] }}$, where $h$ is the homomorphism from $J_{p}$ to $\operatorname{chase}\left(I_{p}, \Sigma\right)$ from the assumption of the lemma:

$$
e(s)= \begin{cases}s, & \text { if } s \text { is a constant } \\ \theta^{[\text {null }]}\left(h\left(\theta^{\overline{[\text { null }]}}(s)\right)\right), & \text { if } s \text { is a null. }\end{cases}
$$

We need to show that this mapping $e$ satisfies the definition of homomorphism: For an arbitrary fact $R(\vec{s}) \in J$, we prove that $R(e(\vec{s})) \in \operatorname{chase}(I, \Sigma)$ holds.

By the definition of canonical chase tree and bijectivity of $\theta^{[\mathrm{tr}]}, \theta$ is a surjective mapping. Thus, for each fact $R(\vec{s}) \in J$, there exists a fact $R\left(\vec{s}^{\prime}\right)$ in $J_{p}$ such that $\theta\left(\vec{s}^{\prime}\right)=\vec{s}$. The existence of the homomorphism $h$ from $J_{p}$ to $\operatorname{chase}\left(I_{p}, \Sigma\right)$ implies that for each $R\left(\vec{s}^{\prime}\right)$ there is a fact $h\left(R\left(\vec{s}^{\prime}\right)\right) \in \operatorname{chase}\left(I_{p}, \Sigma\right)$. Consider the chase forest $\mathcal{F}_{\Sigma, I_{p}}$ of $I_{p}$ with $\Sigma$ and let $\left(\tau_{j}, \overrightarrow{b_{0}}, \vec{b}\right)$ be the triggering of some part $\tau_{j}$ in a nested tgd of $\Sigma$ that has produced the atom $h\left(R\left(\vec{s}^{\prime}\right)\right)$. Since $\theta$ is a c-homomorphism from $I_{p}$ to $I$, there also exists a triggering $\left(\tau_{j}, \theta\left(\vec{b}_{0}\right), \theta(\vec{b})\right)$ in the chase forest $\mathcal{F}_{\Sigma, I}$ of $I$ producing the fact $\theta\left(h\left(R\left(\vec{s}^{\prime}\right)\right)\right) \in \operatorname{chase}(I, \Sigma)$. These facts and mappings are presented in Figure 6. 


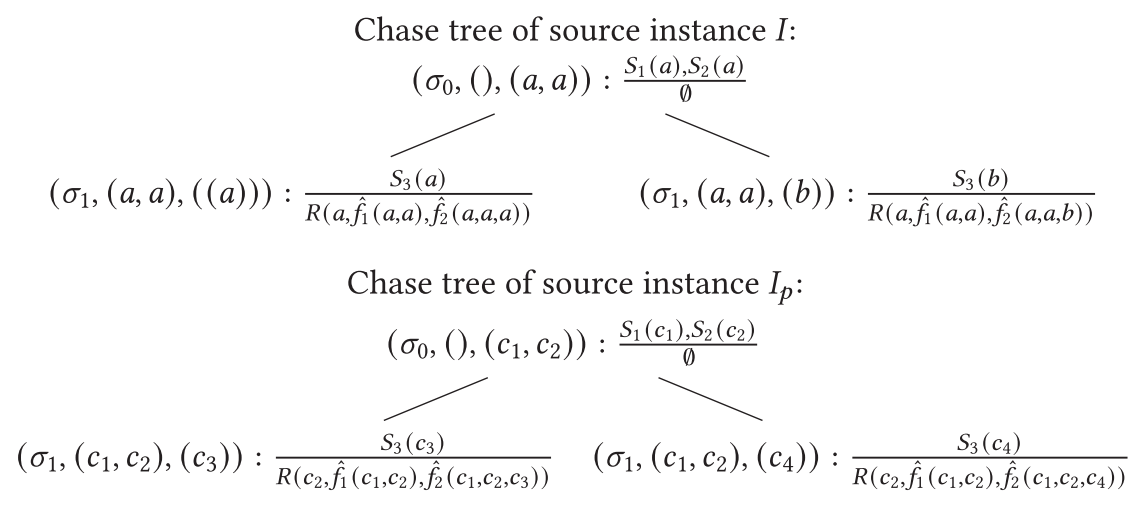

Fig. 7. Chase tree of $I$ in Example 3.21 (upper part) and its canonical chase tree of $I_{p}$ (lower part).

We have shown that for an arbitrary fact $R\left(\vec{s}^{\prime}\right) \in J_{p}$ such that $\theta\left(\vec{s}^{\prime}\right)=\vec{s}$, there exists a fact $\theta\left(h\left(R\left(\vec{s}^{\prime}\right)\right)\right)$ in chase $(I, \Sigma)$. It remains to show that this fact coincides with $R(e(\vec{s}))$. Recall that $e$ preserves constants and maps each null $s_{i}$ to $\theta^{[\text {null }]}\left(h\left(\theta^{\overline{[n u l l]}}\left(s_{i}\right)\right)\right)$. For each term position $i \leq\left|\vec{s}^{\prime}\right|$ and for the $i$ th element $s_{i}^{\prime}$ of $\vec{s}^{\prime}$, consider two cases:

Case 1: $s_{i}^{\prime}$ is a null. Then, also the corresponding element $s_{i}$ of $\vec{s} \in \operatorname{dom}(J)$ is a null. Then, $\theta\left(h\left(s_{i}^{\prime}\right)\right)=\theta\left(h\left(\theta^{\overline{[\text { null }]}}\left(s_{i}\right)\right)\right)=\theta^{[\text {null }]}\left(h\left(\theta^{\overline{[\text { null] }}}\left(s_{i}\right)\right)\right)$ holds, and thus $\theta\left(h\left(s_{i}^{\prime}\right)\right)=e\left(s_{i}\right)$.

Case 2: $s_{i}^{\prime}$ is a constant. By the choice of $\vec{s}^{\prime}$, the equality $\theta\left(s_{i}^{\prime}\right)=s_{i}$ holds. Note also that $h$ preserves constants, so $\theta\left(h\left(s_{i}^{\prime}\right)\right)=\theta\left(s_{i}^{\prime}\right)=s_{i}=e\left(s_{i}\right)$.

We have demonstrated that, for an arbitrary fact $R(\vec{s}) \in J$, there exists a fact $R(e(\vec{s}))$ in chase $(I, \Sigma)$ and thus $e$ is a homomorphism from $J$ to $\operatorname{chase}(I, \Sigma)$.

Example 3.21. Consider the nested tgds $\sigma$ and $\sigma^{\prime}$ :

$$
\begin{aligned}
& \sigma: \forall x_{1} \forall x_{2}\left(S_{1}\left(x_{1}\right) \wedge S_{2}\left(x_{2}\right) \rightarrow \exists y\left(\forall x_{3}\left(S_{3}\left(x_{3}\right) \rightarrow \exists z\left(R\left(x_{2}, y, z\right)\right)\right)\right),\right. \\
& \sigma^{\prime}: \forall x_{1}\left(S_{1}\left(x_{1}\right) \rightarrow \exists y\left(\forall x_{2} \forall x_{3}\left(S_{2}\left(x_{2}\right) \wedge S_{3}\left(x_{3}\right) \rightarrow \exists z\left(R\left(x_{2}, y, z\right)\right)\right)\right),\right.
\end{aligned}
$$

with the Skolemized forms given below:

$$
\begin{aligned}
& \sigma: \forall x_{1} \forall x_{2}\left(S_{1}\left(x_{1}\right) \wedge S_{2}\left(x_{2}\right) \rightarrow \forall x_{3}\left(S_{3}\left(x_{3}\right) \rightarrow R\left(x_{2}, f_{1}\left(x_{1}, x_{2}\right), f_{2}\left(x_{1}, x_{2}, x_{3}\right)\right)\right)\right), \\
& \sigma^{\prime}: \forall x_{1}\left(S_{1}\left(x_{1}\right) \rightarrow\left(\forall x_{2} \forall x_{3}\left(S_{2}\left(x_{2}\right) \wedge S_{3}\left(x_{3}\right) \rightarrow R\left(x_{2}, g_{1}\left(x_{1}\right), g_{2}\left(x_{1}, x_{2}, x_{3}\right)\right)\right)\right) .\right.
\end{aligned}
$$

Suppose that we want to test $\Sigma \mid=\sigma$ with $\Sigma=\left\{\sigma^{\prime}\right\}$ and consider the source instance $I=$ $\left\{S_{1}(a), S_{1}(b), S_{2}(a), S_{2}(b), S_{3}(a), S_{3}(b)\right\}$. Figure 7 shows a chase tree $T$ of source instance $I$ with nested tgds $\sigma$ and the corresponding canonical chase tree $T_{p}$. In these trees, apart from the triggerings, we also display the relevant source atoms enabling each triggering (above the bar) and the target atoms produced by each triggering (below the bar). In case of $T_{p}$, these are the canonical source and target instances, respectively. In particular, we have

$I_{p}=\left\{S_{1}\left(c_{1}\right), S_{2}\left(c_{2}\right), S_{3}\left(c_{3}\right), S_{3}\left(c_{4}\right)\right\}$,

$J_{p}=\left\{R\left(c_{2}, \hat{f}_{1}\left(c_{1}, c_{2}\right), \hat{f}_{2}\left(c_{1}, c_{2}, c_{3}\right)\right), R\left(c_{2}, \hat{f}_{1}\left(c_{1}, c_{2}\right), \hat{f}_{2}\left(c_{1}, c_{2}, c_{4}\right)\right)\right\}$, and

$J=\left\{R\left(a, \hat{f}_{1}(a, a), \hat{f}_{2}(a, a, a)\right), R\left(a, \hat{f}_{1}(a, a), \hat{f}_{2}(a, a, b)\right)\right\}$.

Moreover, we have $\operatorname{chase}\left(I_{p}, \Sigma\right)=\left\{R\left(c_{2}, \hat{g}_{1}\left(c_{1}\right), \hat{g}_{2}\left(c_{1}, c_{2}, c_{3}\right)\right), R\left(c_{2}, \hat{g}_{1}\left(c_{1}\right), \hat{g}_{2}\left(c_{1}, c_{2}, c_{4}\right)\right)\right\}$. Hence, there exists a homomorphism $h: J_{p} \rightarrow \operatorname{chase}\left(I_{p}, \Sigma\right)$ with $h=\left[\hat{f}_{1}\left(c_{1}, c_{2}\right) \mapsto \hat{g}_{1}\left(c_{1}\right), \hat{f}_{2}\left(c_{1}, c_{2}, c_{3}\right) \mapsto\right.$ $\left.\hat{g}_{2}\left(c_{1}, c_{2}, c_{3}\right), \hat{f}_{2}\left(c_{1}, c_{2}, c_{4}\right) \mapsto \hat{g}_{2}\left(c_{1}, c_{2}, c_{4}\right)\right]$. 
The bijection $\theta^{[\mathrm{tr}]}$ between the three triggerings in $T_{p}$ and the three triggerings in $T$ is clear. The c-homomorphism $\theta$ according to the proof of Lemma 3.18 is $\theta=\left[c_{1} \mapsto a, c_{2} \mapsto a, c_{3} \mapsto a, c_{4} \mapsto b\right]$ and thus $\theta^{\text {[null }]}=\left[\hat{f}_{1}\left(c_{1}, c_{2}\right) \mapsto \hat{f}_{1}(a, a), \hat{f}_{2}\left(c_{1}, c_{2}, c_{3}\right) \mapsto \hat{f}_{2}(a, a, a), \hat{f}_{2}\left(c_{1}, c_{2}, c_{4}\right) \mapsto \hat{f}_{2}(a, a, b)\right]$. From this, we define the mapping $e: \operatorname{dom}(J) \rightarrow \operatorname{dom}(\operatorname{chase}(I, \Sigma))$ as in the proof of Lemma 3.18:

$$
e(s)= \begin{cases}s, & \text { if } s \text { is a constant } \\ \left.\theta^{\text {[null }]}\left(h\left(\theta^{\overline{\text { null }}}\left(\hat{f}_{1}(a, a)\right)\right)\right)=\theta^{[\text {null }}\left(h\left(\hat{f}_{1}\left(c_{1}, c_{2}\right)\right)\right)\right)=\hat{g}_{1}(a), & \text { if } s=\hat{f}_{1}(a, a) . \\ \theta^{[\text {null }]}\left(h\left(\theta^{\overline{\text { null }]}}\left(\hat{f}_{2}(a, a, a)\right)\right)\right)=\hat{g}_{2}(a, a, a), & \text { if } s=\hat{f}_{2}(a, a, a) . \\ \theta^{[\text {null }]}\left(h\left(\theta^{\overline{\text { null }}}\left(\hat{f}_{2}(a, a, b)\right)\right)\right)=\hat{g}_{2}(a, a, b), & \text { if } s=\hat{f}_{2}(a, a, b) .\end{cases}
$$

Moreover, chase $(I, \Sigma)$ with $\Sigma=\left\{\sigma^{\prime}\right\}$ contains eight atoms obtained by instantiating any of the variables $x_{1}, x_{2}, x_{3}$ in $\sigma^{\prime}$ to either $a$ or $b$. In particular, $\left\{R\left(a, \hat{g}_{1}(a), \hat{g}_{2}(a, a, a)\right), R\left(a, \hat{g}_{1}(a), \hat{g}_{2}(a, a, b)\right)\right\} \subseteq$ chase $(I, \Sigma)$ holds. Hence, $e$ is indeed a homomorphism from $J$ to chase $(I, \Sigma)$.

Lemma 3.18 reduced checking $\operatorname{chase}(I, \sigma) \rightarrow \operatorname{chase}(I, \Sigma)$ to a series of tests of $J_{p} \rightarrow \operatorname{chase}\left(I_{p}, \Sigma\right)$, for the canonical source and target instances $I_{p}$, respectively, $J_{p}$, of each pattern $p$, realized in the chase forest of $I$ with $\sigma$. The next Lemma 3.22 strengthens this result by showing that only patterns up to a certain size (which is independent of a concrete source instance $I$ ) need to be considered.

LEMMA 3.22. Consider the following setting:

- A set $\Sigma$ of nested tgds and a nested $\operatorname{tg} d \sigma$.

- Integer $k>v_{\sigma} w_{\Sigma}$, where $v_{\sigma}$ is the number of existentially quantified variables (i.e., distinct Skolem functions) in $\sigma$ and $w_{\Sigma}$ is the maximum number of universally quantified variables in any nested tgd in $\Sigma$.

- Patterns $p^{k}, p$ of $\sigma$ where $p^{k}$ is a $k$-pattern and $p^{k} \leq_{k} p$ holds.

- Pairs $\left(I_{p^{k}}, J_{p^{k}}\right)$ and $\left(I_{p}, J_{p}\right)$ of the canonical source and target instances of the patterns $p^{k}$ and $p$, respectively. We assume $I_{p^{k}} \subseteq I_{p}$ and $J_{p^{k}} \subseteq J_{p}$, that is, the same constants as in $\left(I_{p^{k}}, J_{p^{k}}\right)$ are used to instantiate the subtree $p^{k}$ of $p$ in $\left(I_{p}, J_{p}\right)$.

Then, every homomorphism $h^{k}$ from $J_{p^{k}}$ to chase $\left(I_{p^{k}}, \Sigma\right)$ can be extended to a homomorphism $h$ from $J_{p}$ to $\operatorname{chase}\left(I_{p}, \Sigma\right)$.

The proof of Lemma 3.22 will require reasoning about parts of canonical instances corresponding to clone subtrees in patterns. To this end, we first formulate an easy lemma that establishes the relationship between such subinstances.

Definition 3.23. Let $J$ be an instance resulting from a chase with nested tgds. For a subinstance $K$ of $J$, we define the set $l \operatorname{cst}(K)$ of local constants of $K$ as $\operatorname{cst}(K) \backslash \operatorname{cst}(J \backslash K)$. That is, local to $K$ are constants occurring in $K$ and nowhere else in $J$, as non-functional terms (i.e., constants) or as arguments of nulls.

LEMMA 3.24. Let $p$ be a pattern of a chase tree $T$ with a nested $\operatorname{tg} d \sigma$ and let $J_{p}$ be its canonical target instance; let $v_{1}, v_{2}$ be child nodes of some node $v$ of $p$ such that the subtrees $t_{1}, t_{2}$ of $p$ rooted at nodes $v_{1}, v_{2}$ are isomorphic; finally, let $K_{1}, K_{2}$ denote the subinstances of $J_{p}$ such that $K_{i}$ consists of the facts generated by any of the triggerings in the subtree $t_{i}$ with $i \in\{1,2\}$. Then $K_{1}$ and $K_{2}$ are equal up to a c-isomorphism (i.e., a bijection that is a c-homomorphism in both directions) that maps constants from lcst $\left(K_{1}\right)$ to lcst $\left(K_{2}\right)$ and leaves all other constants unchanged.

Proof. The proof is straightforward.

With Lemma 3.24, we can now prove Lemma 3.22 about extensions of homomorphisms to canonical instances resulting from $k^{+}$-increments. 
Proof of Lemma 3.22. The proof is by induction on the length $i$ of the sequence of $k^{+}-$ increments of $p^{k}$ transforming it to $p$. For the base case $i=0$, the existence of $h$ is an assumption of the lemma. Take as induction hypothesis that for some $i \geq 0$, a homomorphism $h_{i}$ from $J_{p_{i}}$ to $\operatorname{chase}\left(I_{p_{i}}, \Sigma\right)$ exists, s.t. $h_{i}$ extends $h^{k}$. We show that $h_{i}$ can be further extended to a homomorphism $h_{i+1}$ from $J_{p_{i+1}}$ to chase $\left(I_{p_{i+1}}, \Sigma\right)$, where $p_{i+1}$ is obtained from $p_{i}$ by a single $k^{+}$-increment.

By the definition of $k^{+}$-increment, $p_{i+1}$ is obtained by inserting a copy of some node $v$ in $p_{i}$-including the full subtree $\pi_{v}$ rooted at $v$-to the parent node of $v$. Moreover, $v$ is one of $\ell$ nodes $v_{1}, \ldots, v_{\ell}$ with $\ell \geq k$ having the same parent in $p_{i}$ and being roots to subtrees each of which is isomorphic to $\pi_{v}$. In the canonical chase tree $T_{p_{i}}$ of $p_{i}$ this corresponds to a parent triggering $t_{\rho}$ of the part label(parent $\left.(v)\right)$, with $\ell \geq k$ isomorphic subtrees rooted at triggerings $t_{1}=\left(\sigma_{i}, \vec{a}_{0}, \vec{a}_{1}\right), \ldots, t_{\ell}=\left(\sigma_{i}, \vec{a}_{0}, \vec{a}_{\ell}\right)$ of the same part $\sigma_{i}$ with the same input assignment $\vec{a}_{0}$.

The canonical chase tree $T_{p_{i+1}}$ extends $T_{p_{i}}$ with yet another subtree rooted at a triggering $t_{\ell+1}=\left(\sigma_{i}, \vec{a}_{0}, \vec{a}_{\ell+1}\right)$ placed directly under $t_{\rho}$. For $j \in\{1, \ldots, \ell+1\}$, we define $K_{j} \subseteq J_{p_{i+1}}$ as $K_{j}=$ $\bigcup\left\{\operatorname{facts}(t) \mid t \in \operatorname{desc}\left(t_{j}\right)\right\}$, i.e., $K_{j} \subseteq J_{p_{i}}$ for each $j \in\{1, \ldots, \ell\}$ and $K_{\ell+1}=J_{p_{i+1}} \backslash J_{p_{i}}$.

Let $F$ denote the set of nulls shared between any two distinct instances in the set $\left\{K_{1}, \ldots, K_{\ell+1}\right\}$. By Lemma 3.14, each such shared null has the form $\hat{f}(\vec{b})$ where $\vec{b}$ is a prefix of the common input assignment $\vec{a}_{0}$ of the triggerings $t_{1}, \ldots, t_{\ell+1}$. Thus, the number of nulls in $F$ is bounded: $|F| \leq v_{\sigma}$. This observation pinpoints the key idea of the proof, formulated as the following claim:

Claim A. There exists at least one $\tilde{K} \in\left\{K_{1}, \ldots, K_{\ell}\right\}$ such that for every null $\hat{f}(\vec{a}) \in F$, with $h_{i}(\hat{f}(\vec{a}))=\hat{g}(\vec{b}), \vec{b}$ does not contain a local constant of $\tilde{K}$. This claim is proved as follows: As explained above, there are at most $v_{\sigma}$ nulls in $F$, and each term in an instance obtained by the chase with $\Sigma$ is built using at most $w_{\Sigma}$ constants, namely: either a single constant or up to $w_{\Sigma}$ constants serving as arguments of a null. Therefore, at most $w_{\Sigma}$ instances among $\left\{K_{1}, \ldots, K_{\ell}\right\}$ can contribute their local constants to the term $h_{i}(\hat{f}(\vec{a}))$, and thus local constants from at most $v_{\sigma} w_{\Sigma}$ instances can occur in $h_{i}(F)$. However, by the assumption of the lemma, $k>v_{\sigma} w_{\Sigma}$. Hence, by $\ell \geq k$, a subinstance $\tilde{K} \in\left\{K_{1}, \ldots, K_{\ell}\right\}$ with the desired property exists.

We now consider a subinstance $\tilde{K} \in\left\{K_{1}, \ldots, K_{\ell}\right\}$ from the claim above and the subinstance $K^{\prime}=K_{\ell+1}$, distinguishing $J_{p_{i+1}}$ from $J_{p_{i}}$. Our task is to extend the homomorphism $h_{i}$ to the nulls of $K^{\prime}$, which are not present in the domain of $J_{p_{i}}$, to obtain a homomorphism $h_{i+1}$ from $J_{p_{i+1}}$ to $\operatorname{chase}\left(I_{p_{i+1}}, \Sigma\right) . \tilde{K}$ and $K^{\prime}$ represent two isomorphic sibling subtrees of the pattern $p_{i+1}$ in its canonical target instance. By Lemma 3.24 a renaming of local constants of $\tilde{K}$ into the local constants of $K^{\prime}$ exists, transforming $\tilde{K}$ into $K^{\prime}$. We let $\theta$ be such a renaming extended with the identity mapping on all other constants in $J_{p_{i}}$. That is, $\theta$ is the identity on $\operatorname{cst}\left(J_{p_{i}}\right) \backslash \operatorname{lcst}(\tilde{K})$. By definition $\theta$ is a bijection between $\operatorname{cst}(\tilde{K})$ and $\operatorname{cst}\left(K^{\prime}\right)$. Consider the function $h_{i+1}^{\prime}$ defined on $\operatorname{dom}\left(K^{\prime}\right)$ as $h_{i+1}^{\prime}(x)=\theta\left(h_{i}\left(\theta^{-1}(x)\right)\right)$. Mapping $h_{i+1}^{\prime}$ can be used to extend $h_{i}$ in the following sense: For every $x \in \operatorname{dom}\left(K^{\prime}\right) \cap \operatorname{dom}\left(J_{p_{i}}\right)$, we have $h_{i+1}^{\prime}(x)=h_{i}(x)$. Indeed, $\theta^{-1}$ preserves the non-local constants of $K^{\prime}$ and by Claim A, $h_{i}$ maps each null containing no local constants of $\tilde{K}$ to a term also using no local constants of $\tilde{K}$ : Thus, by construction of $\theta$ for every null $\hat{f}(\vec{a})$ in the domain of $h_{i}$, we have $\theta\left(h_{i}\left(\theta^{-1}(\hat{f}(\vec{a}))\right)\right)=h_{i}(\hat{f}(\vec{a}))$. Moreover, for a constant $a$, we have $\theta\left(h_{i}\left(\theta^{-1}(a)\right)\right)=\theta\left(\theta^{-1}(a)\right)=a$.

We now take $h_{i+1}=h_{i} \cup h_{i+1}^{\prime}$ and show that $h_{i+1}$ is a homomorphism. First note that $\theta$ is a c-homomorphism from $I_{p_{i}}$ to $I_{p_{i+1}}$. Extended to nulls as in Definition 3.19, $\theta$ is also a chomomorphism from $J_{p_{i}}$ to $J_{p_{i+1}}$ and from $\operatorname{chase}\left(I_{p_{i}}, \Sigma\right)$ to $\operatorname{chase}\left(I_{p_{i+1}}, \Sigma\right)$, analogously to the proof of Lemma 3.18. In particular, $\theta\left(h_{i}(\tilde{K})\right) \subseteq \operatorname{chase}\left(I_{p_{i+1}}, \Sigma\right)$ and, since $\tilde{K}=\theta^{-1}\left(K^{\prime}\right)$, we have $\theta\left(h_{i}\left(\theta^{-1}\left(K^{\prime}\right)\right)\right) \subseteq$ chase $\left(I_{p_{i+1}}, \Sigma\right)$. Note that $h_{i}$ preserves constants, and therefore $h_{i+1}^{\prime}=\theta^{-1} \circ h_{i} \circ \theta$ is a constant-preserving homomorphism from $K^{\prime}$ to $\operatorname{chase}\left(I_{p_{i+1}}, \Sigma\right)$. Since $h_{i+1}=h_{i} \cup h_{i+1}^{\prime}$, we have 
just shown that $h_{i+1}$ is a homomorphism from $K^{\prime}$ to $\operatorname{chase}\left(I_{p_{i+1}}, \Sigma\right)$ and with that, a homomorphism from $J_{p_{i+1}}$ to $\operatorname{chase}\left(I_{p_{i+1}}, \Sigma\right)$, as desired.

We now have all properties required to prove Theorem 3.1:

THEOREM 3.1. The implication problem for nested tgds is decidable.

Proof. The decision procedure ImPLiEs relies on the usual characterization of the implication in the finite based on the chase, namely, $\Sigma=\sigma$ if and only if $\operatorname{chase}(I, \sigma) \rightarrow \operatorname{chase}(I, \Sigma)$ holds for every source instance $I$. This characterization is applicable to mappings that are closed under target homomorphisms, which is the case for SO tgds [11], and hence, in particular for nested tgds. Indeed, assume $\Sigma \mid=\sigma$. That is, for each pair $(I, J) \mid=\Sigma$, also $(I, J) \mid=\sigma$ is the case. In other terms, each solution $J$ for $I$ under $\Sigma$ is also a solution for $I$ under $\sigma$ and chase $(I, \sigma) \rightarrow J$ must hold. In particular, this holds for $J=\operatorname{chase}(I, \Sigma)$, and therefore chase $(I, \sigma) \rightarrow \operatorname{chase}(I, \Sigma)$ is the case. In the opposite direction, assume that for all source instances $I$, a homomorphism from chase $(I, \sigma)$ to chase $(I, \Sigma)$ exists. Since chase $(I, \Sigma)$ is a universal solution under $\Sigma$, for arbitrary $J$ such that $(I, J)$ $\Sigma$, chase $(I, \Sigma) \rightarrow J$ and thus chase $(I, \sigma) \rightarrow J$ holds. Since $\sigma$ is closed under target homomorphisms, an arbitrary solution $J$ for $I$ under $\Sigma$ is also a solution for $I$ under $\sigma$, for arbitrary $I$. This concludes the proof of the characterization of implication in the finite via the chase.

The completeness of the decision procedure is immediate: $\Sigma \not \sigma$ is only returned if for some $k$-pattern $p^{k}$ with the canonical source, respectively, target instances $I_{p^{k}}, J_{p^{k}}$, no homomorphism from $J_{p^{k}}$ to $\operatorname{chase}\left(I_{p^{k}}, \Sigma\right)$ exists. Note that $J_{p^{k}}$ is a subinstance of $\operatorname{chase}\left(I_{p^{k}}, \sigma\right)$, and thus $\operatorname{chase}\left(I_{p^{k}}, \sigma\right) \nrightarrow \operatorname{chase}\left(I_{p^{k}}, \Sigma\right)$ holds as well. This makes $I_{p^{k}}$ a witness for the failure of the implication.

It remains to show the soundness. Assume that for some nested $\operatorname{tgd} \sigma$ and a set $\Sigma$ of nested tgds the procedure returns true. Consider an arbitrary source instance $I$ corresponding to the target instance $J_{\sigma}=\operatorname{chase}(I, \sigma)$. We show that a homomorphism from $J_{\sigma}$ to $J_{\Sigma}$ exists, where $J_{\Sigma}=\operatorname{chase}(I, \Sigma)$. By Lemma 3.18, it suffices to show that for each pattern $p$ in the chase forest of $I$ with $\sigma$, a homomorphism $e$ from $J_{p}$ to $\operatorname{chase}\left(I_{p}, \Sigma\right)$ exists, where $I_{p}, J_{p}$ are the canonical source and target instances of $p$, respectively.

Let $p^{k}$ be a $k$-pattern of $\sigma$ such that $p^{k} \leq_{k} p$ holds. By Lemma 3.17, such $k$-pattern $p^{k}$ exists. Since the procedure IMPLIES returned true, it follows that a homomorphism $h^{k}$ from $J_{p^{k}}$ to chase $\left(I_{p^{k}}, \Sigma\right)$ exists, where $I_{p^{k}}$ and $J_{p^{k}}$ are, respectively, the canonical source and target instances of $p^{k}$.

We now apply Lemma 3.22 to prove that $h^{k}$ can be extended to a homomorphism $h$ from $J_{p}$ to chase $\left(I_{p}, \Sigma\right)$, where $I_{p}, J_{p}$ are, respectively, the canonical source and target instances of $p$. Since $p$ is arbitrary, by Lemma 3.18 this implies that a homomorphism from chase $(I, \sigma)$ to $\operatorname{chase}(I, \Sigma)$ exists for every source instance $I$ and, hence, $\Sigma \mid=\sigma$.

We conclude this section by discussing an immediate consequence of Theorem 3.1.

\section{COROLlary 3.25. The logical equivalence problem for nested tgds is decidable.}

In contrast, it is known that the logical equivalence problem for SO tgds is undecidable, according to Theorem 1 in Reference [12], which builds on Reference [3]. As a matter of fact, an examination of the proof of Theorem 1 in Reference [12] reveals that the following problem is undecidable: Given an SO tgd $\sigma$ and a finite set $\Sigma^{\prime}$ of s-t tgds, is $\sigma \equiv \Sigma^{\prime}$ ? Hence, the following problem is undecidable as well: Given an SO $\operatorname{tgd} \sigma$ and a finite set $\Sigma^{\prime}$ of nested tgds, is $\sigma \equiv \Sigma^{\prime}$ ? Therefore, Corollary 3.25 contributes to the delineation of the boundary between decidability and undecidability for the logical equivalence problem. 


\section{THE STRUCTURE OF THE CORE AND APPLICATIONS}

As mentioned in Section 2, the class of nested GLAV mappings contains the class of GLAV mappings and is, in turn, contained in the class of (mappings specified by) plain SO tgds. Moreover, it is known that both containments are proper. In this section, we produce powerful tools that allow us to tell apart nested GLAV mappings from GLAV mappings, and also plain SO tgds from nested GLAV mappings. The main result of this section is an algorithm for telling whether or not a given nested GLAV mapping is logically equivalent to a GLAV mapping. In addition, we give useful sufficient conditions for showing that a plain SO tgd is not logically equivalent to any nested GLAV mapping. Moreover, these conditions are easy to apply in concrete instances, in the sense that they give the user tools based on simple and natural concepts.

The results in this section are obtained by analyzing the structure of the core of the universal solutions of nested GLAV mappings. We embark on this analysis next, which we believe is of interest in its own right.

\subsection{Nested GLAV Mappings vs. GLAV Mappings}

Recall that every schema mapping $\mathcal{M}$ specified by an SO-tgd admits universal solutions. Moreover, for every source instance $I$, a canonical universal solution chase $(I, \mathcal{M})$ for $I$ w.r.t. $\mathcal{M}$ can be obtained via the chase procedure. Since all universal solutions for a given source instance $I$ are homomorphically equivalent, it follows that their cores are unique up to isomorphism, hence, we can take core $(\operatorname{chase}(I, \mathcal{M}))$ as the core of the universal solutions for $I$ w.r.t. $\mathcal{M}$ [10]. Note that, in general, $\operatorname{core}(\operatorname{chase}(I, \mathcal{M}))$ need not be a universal solution for $I$ w.r.t. $\mathcal{M}$ [9]. However, if $\mathcal{M}$ is specified by a plain SO tgd, then $\operatorname{core}(\operatorname{chase}(I, \mathcal{M}))$ is a universal solution for $I$ w.r.t. $\mathcal{M}$. The reason for this is that, as shown in Reference [2], every schema mapping $\mathcal{M}$ specified by a plain SO tgd is closed under target homomorphisms, which means that if $J$ is a solution for $I$ w.r.t. $\mathcal{M}$ and if there is a homomorphism from $J$ to $J^{\prime}$ that is the identity on constants, then $J^{\prime}$ is also a solution for $I$ w.r.t. $\mathcal{M}$. Moreover, $\operatorname{core}(\operatorname{chase}(I, \mathcal{M}))$ is the smallest universal solution for $I$ w.r.t. $\mathcal{M}$. In particular, the above facts hold true for nested GLAV mappings (hence, also for GLAV mappings). We will make extensive use of the following notion, which was introduced in Reference [9].

Definition 4.1. A schema mapping $\mathcal{M}$ specified by an SO tgd has bounded f-block size if there is an integer $b$ such that for every source instance $I$, the f-block size of core $(\operatorname{chase}(I, \mathcal{M}))$ is at most $b$, the smallest such $b$ is then called the f-block size of $\mathcal{M}$; otherwise, we say that $\mathcal{M}$ has unbounded f-block size.

The next result follows immediately from Proposition 3.14 and Theorem 4.10 in Reference [9]. Recall that every nested GLAV mapping is equivalent to some plain SO tgd.

Theorem 4.2 ([9]). A schema mapping $\mathcal{M}$ specified by a plain SO tgd is logically equivalent to a GLAV schema mapping if and only if $\mathcal{M}$ has bounded f-block size.

Proof. Theorem 4.10 [9] states: A schema mapping $\mathcal{M}$ specified by an $S O$ tgd is CQ-equivalent to a GLAV schema mapping if and only if $\mathcal{M}$ has bounded f-block size. This is almost the statement we need, except that it talks about CQ-equivalence instead of logical equivalence. However, as has been detailed on page four in Section 1, by combining results from References [2] and [9], we may conclude that CQ-equivalence and logical equivalence coincide for plain SO tgds.

The preceding Theorem 4.2 will be used to prove the main result in this section, which we now state formally.

Theorem 4.3. The following problem is decidable: Given a nested GLAV mapping $\mathcal{M}$, is there a GLAV mapping $\mathcal{M}^{\prime}$ such that $\mathcal{M}$ is logically equivalent to $\mathcal{M}^{\prime}$ ? 
In view of Theorem 4.2, it suffices to give an algorithm that, given a nested GLAV schema mapping $\mathcal{M}$, determines whether or not $\mathcal{M}$ has bounded f-block size. To this end, we introduce a crucial property of mappings and show that nested GLAV mappings have this property.

Definition 4.4. Let $C$ be a class of schema mappings. We say that $C$ has effective threshold for $f$-block size if there exists a recursive function $f: C \rightarrow \mathbb{N}$, where $\mathbb{N}$ is the set of natural numbers, s.t. every mapping $\mathcal{M} \in C$ either has f-block size at most $f(\mathcal{M})$ or has unbounded f-block size. $\triangleleft$

Before showing that nested GLAV mappings actually have effective bounded threshold for fblock size, let us give the following lemma and introduce some useful notation:

Lemma 4.5. For every nested GLAV mapping specified by a set $\Sigma$ of nested tgds, there exists a single nested $\operatorname{tg} d \sigma$, such that $\Sigma$ and $\sigma$ have the same f-block size. Moreover, for every source instance I for $\Sigma$, there is a source instance $I^{\prime}$ for $\sigma$ with $\left|I^{\prime}\right|=|I|+1$, such that chase $(I, \Sigma)=\operatorname{chase}\left(I^{\prime}, \sigma\right)$.

Proof. Let $\Sigma=\left\{\sigma_{i} \mid 1 \leq i \leq k\right\}$ be a set of nested tgds. Let $R$ denote a relation symbol not occurring in $\Sigma$ and let $x$ be a variable not occurring in $\Sigma$. Then $\sigma$ is given by

$$
R(x) \rightarrow\left(\sigma_{1} \wedge \ldots \wedge \sigma_{k}\right) .
$$

We now claim that $\Sigma$ and $\sigma$ have the same f-block size. Indeed, since $R$ is a fresh relation symbol, the source instance $I$ for $\Sigma$ witnessing some f-block size can be extended to $I^{\prime}=I \cup\{R(a)\}$ for $\sigma$ where $a$ is an arbitrary constant. Thus, we know that $\sigma$ has at least the f-block size of $\Sigma$. Conversely, $\sigma$ has at most the f-block size of $\Sigma$, since the variable $x$ does not occur anywhere in $\Sigma$, thus not allowing f-blocks to be connected through a shared variable.

In Section 3, we introduced several crucial notions for the analysis of nested tgds, such as chase forests, chase trees, patterns, isomorphic subtrees in a pattern, $k$-patterns, and so on. To prove that the class of nested GLAV mappings has effective threshold for f-block size, we have to relativize these notions to a subinstance of the chase result.

Definition 4.6 (Chase tree with restricted conclusions). Let $\sigma$ be a nested tgd and let $I$ be a source instance. Let $\mathcal{F}_{\sigma, I}$ be the chase forest of $I$ with $\sigma$ and let $T_{\sigma, I}=(V, T)$ be a chase tree in this chase forest. Moreover, let $J \subseteq$ chase $(I, \sigma)$. Recall from Definition 3.3 that each vertex in $T_{\sigma, I}$ corresponds to a triggering $\left(\sigma_{i}, \vec{a}_{0}, \vec{a}\right)$ and that $\sigma_{i}$ is a Skolemized formula of the form $\forall \vec{x}\left(\varphi\left(\vec{x}_{0}, \vec{x}\right) \rightarrow \alpha_{1}\left(\vec{x}_{0}, \vec{x}\right) \wedge\right.$ $\left.\cdots \wedge \alpha_{n}\left(\vec{x}_{0}, \vec{x}\right)\right)$.

The chase tree with conclusions restricted to $J$ (or, simply, the $R C$-chase tree w.r.t. J), which we denote as $T_{\sigma, I}(J)$, is obtained from $T_{\sigma, I}$ by leaving the tree structure unchanged and extending the triples $\left(\sigma_{i}, \vec{a}_{0}, \vec{a}\right)$ in the vertices with the fourth element $C$, defined as the following set of conclusion atoms of the part $\sigma_{i}: C=\left\{\alpha_{i}\left(\vec{x}_{0}, \vec{x}\right) \mid \mu\left(\alpha_{i}\left(\vec{a}_{0}, \vec{a}\right)\right) \in J\right\}$, where $\mu\left(\alpha_{i}\left(\vec{a}_{0}, \vec{a}\right)\right)$ denotes the fact obtained from the atom $\alpha_{i}\left(\vec{a}_{0}, \vec{a}\right)$ by replacing every Skolem term $f\left(\vec{a}_{0}, \vec{a}\right)$ in it by the null $\mu\left(f\left(\vec{a}_{0}, \vec{a}\right)\right)=\hat{f}\left(\vec{a}_{0}, \vec{a}\right)$.

In other words, the additional label $C$ of each vertex in an RC-chase tree adds to the chase tree the information as to which target atoms are ultimately retained in the subset $J$ of $\operatorname{chase}(I, \sigma)$. We now also extend the notion of patterns from Definition 3.5 to allow for restrictions of the conclusions.

Definition 4.7 (Patterns with restricted conclusions). Let $\sigma$ be a nested tgd and let $p$ be a pattern of $\sigma$. Recall that a pattern is a rooted tree where each vertex is labeled by some part $\sigma_{i}$ of $\sigma$. A pattern with restricted conclusions (or, simply, an $R C$-pattern) obtained from $p$ has the same tree structure as $p$, and the label $\sigma_{i}$ of each vertex is extended to the label $\left(\sigma_{i}, C\right)$, where $C$ is an arbitrary subset of the conclusion atoms $\left\{\alpha_{1}\left(\vec{x}_{0}, \vec{x}\right), \ldots, \alpha_{n}\left(\vec{x}_{0}, \vec{x}\right)\right\}$ of $\sigma_{i}$. 
Let $I$ be an arbitrary source instance and let $J \subseteq \operatorname{chase}(I, \sigma)$ such that all atoms in $J$ are generated by a single chase tree $T_{\sigma, I}$. Then the $R C$-pattern w.r.t. $J$ is the RC-pattern obtained from $T_{\sigma, I}(J)$ by leaving the tree structure unchanged and restricting every label $\left(\sigma_{i}, \vec{a}_{0}, \vec{a}, C\right)$ to $\left(\sigma_{i}, C\right)$.

Analogously to Definition 3.6, we can consider the notions of (isomorphic) subtrees of an RCpattern and clones of subtrees, which lead to the definition of RC- $k$-patterns.

Definition 4.8 (RC-k-patterns). Let $\sigma$ be a nested tgd and let $p$ be an RC-pattern of $\sigma$. Let $(V, T)$ denote the tree structure of $p$, and let $\lambda$ denote the vertex labeling of $p$. As in Definition 3.6, we mean by a "subtree" a complete subtree with all its descendant nodes. Now let $v$ be some vertex in $V$ and let $t_{1}=\left(V_{1}, T_{1}\right)$ and $t_{2}=\left(V_{2}, T_{2}\right)$ denote two subtrees of $(V, T)$ rooted at two different child nodes of $v$. We call $t_{1}$ and $t_{2}$ isomorphic if they have the same tree structure and the same labeling, i.e.: there exists an isomorphism $h$ from $\left(V_{1}, T_{1}\right)$ to $\left(V_{2}, T_{2}\right)$ such that $\lambda(v)=\lambda(h(v))$ holds for every $v \in V_{1}$. If this is the case, we also say that $t_{2}$ is an $R C$-clone of $t_{1}$.

Let RC- $C_{t}$ denote the set containing some subtree $t$ and all its RC-clones in $p$. If for each subtree $t$ of $p,\left|\mathrm{RC}-C_{t}\right| \leq k$, we call $p$ an $R C$ - $k$-pattern. RC- $\mathcal{P}_{k}(\sigma)$ is the set of all RC- $k$-patterns of $\sigma . \triangleleft$

Recall from Proposition 3.8 that for a positive integer $k$ and a nested $\operatorname{tgd} \sigma$, the set of $k$-patterns of $\sigma$ is finite. The proof was by structural induction on $\sigma$ making use of the observation, that the sets of $k$-patterns of immediate subparts $\sigma_{1}^{\prime}, \ldots, \sigma_{n}^{\prime}$ of some part $\sigma_{i}$ in $\sigma$ can only be combined in finitely (but of course exponentially) many ways into $k$-patterns of $\sigma_{i}$. For RC-patterns, we get an additional exponentiality, since, in addition to the parts of $\sigma$, we also have to take subsets of the conclusions of each part into account. However, this does not affect the finiteness of RC- $\mathcal{P}_{k}$. We thus get the following result:

COROLLARY 4.9. For a positive integer $k$ and a nested $\operatorname{tg} d \sigma$, the set $R C-\mathcal{P}_{k}$ of $R C$ - $k$-patterns of $\sigma$ is finite.

The definition of the canonical source instance $I_{p}$ of a pattern (see Definition 3.9) is not affected by the restriction of the conclusions in an RC-pattern $p$. The same applies to the canonical chase tree (canonical RC-chase tree), with the provision that the triggerings at its vertices are now specified by quadruples $t=\left(\sigma_{i}, \vec{a}_{0}, \vec{a}, C\right)$ rather than triples $\left(\sigma_{i}, \vec{a}_{0}, \vec{a}\right)$. The function facts $(t)$ applied to such a quadruple $t$ returns the instantiation of atoms in $C$ by $\vec{a}_{0}$ and $\vec{a}$. The canonical target instance $J_{p}$ of the RC-pattern $p$ with the canonical chase tree $(V, T)$ is the union $\bigcup_{\left(\sigma_{i}, \vec{a}_{0}, \vec{a}, C\right) \in V} \operatorname{facts}\left(\left(\sigma_{i}, \vec{a}_{0}, \vec{a}, C\right)\right)$.

Let $T$ be a subtree of an RC-pattern and $\tau=\left(V^{\prime}, T^{\prime}\right)$ be the respective subtree of the canonical chase tree. By the set of facts generated by $T$ (respectively, by $\tau$ ), we mean the set $K=\bigcup\{$ facts $(t) \mid$ $\left.t \in V^{\prime}\right\}$, that is, the set of facts generated by triggerings in $\tau$, restricted to the target atoms in the sets $C$ at each vertex in $\tau$. The set of local constants of $T$ (respectively, of $\tau$ ) is

$$
\operatorname{lcst}(T)=\operatorname{lcst}(\tau)=\left\{a \mid \text { there exists } \vec{a} \text { such that } a \text { occurs in } \vec{a} \text { and }\left(\sigma_{i}, \vec{a}_{0}, \vec{a}, C\right) \in V^{\prime}\right\},
$$

that is, the set of constants occurring in the assignments of the triggerings in $\tau$. Regarding $K$ as a subset of the canonical target instance $J_{p}$ of the RC-pattern $p$, observe that $l \operatorname{cst}(T) \cap \operatorname{cst}(K)$ (the local constants of $T$ actually occurring in $K$ ) are exactly the local constants of $K$ in the sense of Definition 3.23, namely, those constants occurring in $K$ and nowhere else in $J_{p}$. We say that a fact $A$ contains a local constant from $\tau$ if at least one constant of $l \operatorname{cst}(\tau)$ occurs in $A$ : $\operatorname{cst}(\{A\}) \cap l \operatorname{cst}(\tau) \neq \emptyset$. Finally, for every such RC-pattern subtree $T$, with the corresponding subtree $\tau$ of the canonical chase tree and the set $K$ of facts generated by $T$, we fix an order of local constants, which allows us to speak of the tuple $\vec{b}$ of local constants of $\tau$ (respectively, of $K$, respectively, of $T$ ). Note that, for each triggering $\left(\sigma_{i}, \vec{a}_{0}, \vec{a}\right)$, the tuple notation $\vec{a}$ assumes an order of the constants in $\vec{a}$ anyway. This 
order of local constants of individual triggerings can be extended to an order of the local constants of $T$ by fixing the order of the nodes in $T$ (e.g., assume top-down, left-to-right order).

Now consider a pattern $p$ and let $K_{1}, K_{2}$ be sets of facts generated by isomorphic subtrees of a pattern such that the roots of these subtrees are siblings in $p$. Moreover, let the respective tuples of local constants be $\vec{b}_{K_{1}}$ and $\vec{b}_{K_{2}}$. Then, the c-homomorphism $\theta$ mapping the $i$ th constant in $\vec{b}_{K_{1}}$ to the $i$ th constant in $\vec{b}_{K_{2}}$ for every $i$ clearly defines a c-isomorphism between $K_{1}$ and $K_{2}$. It is convenient to denote this c-isomorphism by $\theta\left(\vec{b}_{K_{1}}\right)=\vec{b}_{K_{2}}$.

\section{THEOREM 4.10. The class of nested GLAV mappings has effective threshold for f-block size.}

Proof. By Lemma 4.5, it suffices to consider mappings defined by a single nested $\operatorname{tgd} \sigma$. We have to show that there exists a recursive function $g$ that, for a given nested $\operatorname{tgd} \sigma$, returns an integer $g(\sigma)$ witnessing bounded f-block size for $\sigma$. The proof proceeds in several steps and is split into three claims. We give the overall picture of the proof here. Detailed proofs of the claims are given in the Appendix.

Given an arbitrary nested $\operatorname{tgd} \sigma$, let $n$ denote the maximum size of all RC-1-patterns of $\sigma$. Here, we define the size of an RC-pattern $p$ as the sum of the cardinalities of the sets $C$ of conclusion atoms in the labels of the vertices in $p$. By the finiteness of RC- $\mathcal{P}_{k}$ according to Corollary 4.9, this maximum exists. We set the threshold $g(\sigma)$ to $g(\sigma)=n+1$. Now suppose that there exists a source instance $I$, s.t. $\operatorname{core}(\operatorname{chase}(I, \sigma))$ has f-block size $m \geq g(\sigma)$. We will show that then there exists a source instance $I^{\prime}$, s.t. core $\left(\operatorname{chase}\left(I^{\prime}, \sigma\right)\right)$ has f-block size $\geq m+1$. Since our construction of $I^{\prime}$ from $I$ can be iterated arbitrarily often, it follows immediately that $\sigma$ has unbounded f-block size.

Considering canonical source instances only. By Corollary 3.15, we know that the facts in some f-block $B$ of core $(\operatorname{chase}(I, \sigma))$ must be generated by a single chase tree in the chase forest $\mathcal{F}_{\sigma, I}$. Now suppose that f-block size $m$ of $\operatorname{core}(\operatorname{chase}(I, \sigma))$ is realized by some f-block $B$ of core $(\operatorname{chase}(I, \sigma))$ and that the facts in $B$ are generated by RC-chase tree $T_{p}$ with RC-pattern $p$. Let $I_{p}$ be the canonical source instance of $p$. Then the following property holds:

Claim 1. The f-block size of $\operatorname{core}\left(\operatorname{chase}\left(I_{p}, \sigma\right)\right)$ is at least $m$.

The proof idea of this claim is as follows: Recall from the proof of Lemma 3.18 that there is a one-to-one correspondence between the triggerings in (and the facts generated by) the chase tree $T_{\sigma, I}$ and the corresponding pattern $p$ equipped with the variable bindings according to the canonical source instance $I_{p}$. We can thus define the set $B_{p}$ of facts in the canonical target instance corresponding to the set $B$ in chase $(I, \sigma)$. It can be shown that $B_{p}$ or a superset thereof must also be an f-block in the core of the canonical target instance. In the remainder of the proof, we may thus assume w.l.o.g. that $I$ is the canonical source instance of $p$.

Isomorphic subtrees in $T_{p}$ with local constants in facts of $B$. By our definition of the size of an RC-pattern $p$ as the total number of conclusion atoms in the labels of $p$ and by Corollary 3.15 recalled above, the size of $p$ yields an upper bound on the cardinality of an f-block whose facts are generated by chase tree $T_{p}$ with pattern $p$. We are assuming that f-block $B$ has cardinality greater than the maximum size of all 1-patterns of $\sigma$. Hence, there exist (at least) two siblings in the pattern $p$ that are the roots of isomorphic subtrees $T_{1}$ and $T_{2}$, such that both $T_{1}$ and $T_{2}$ generate some facts of $B$. We strengthen this observation to the following claim:

Claim 2. For each $i \in\{1,2\}$, there exists an atom $A_{i}$ generated by $T_{i}$ such that $A_{i} \in B$ and $A_{i}$ contains a local constant of $T_{i}$. (Recall that in a canonical target instance, local constants do not occur outside a subinstance generated by a subtree of a chase tree.) 
By the considerations on the size of RC-1-patterns and of $B$, it is clear that $p$ must be a $k$-pattern with $k \geq 2$, i.e., there must exist isomorphic subtrees $T_{1}$ and $T_{2}$ whose roots are siblings and which generate some facts of $B$. For the proof of Claim 2, it only remains to show that it cannot happen that the facts generated by $T_{1}$ and $T_{2}$ contain no local constants. Indeed, this can be excluded by showing that otherwise there exists an endomorphism on core (chase $(I, \sigma))$ that allows us to "shrink" $B$ by mapping all facts generated by $T_{1}$ to the facts generated by $T_{2}$ or vice versa. But this contradicts the assumption that $B$ is an f-block in $\operatorname{core}(\operatorname{chase}(I, \sigma))$.

Construct another isomorphic subtree. Let $\vec{b}$ and $\vec{c}$ denote the tuples of the local constants of $T_{1}$ and of $T_{2}$, respectively. By the above considerations, there exists a one-to-one correspondence between these vectors of local constants. We now introduce a vector of fresh constants $\vec{d}$ such that we again have a one-to-one correspondence between this vector and $\vec{b}$. Let $\theta$ realize this oneto-one correspondence, i.e.: $\theta\left(b_{i}\right)=d_{i}$ for every $b_{i}$ in $\vec{b}$ and $d_{i}$ in $\vec{d}$. We extend $I$ to $I^{\prime}=I \cup \theta(I)$. Hence, the chase tree of $I^{\prime}$ with $\sigma$ is obtained from the chase tree of $I$ by adding another isomorphic subtree $T_{3}$ to the RC-pattern $p$ and taking $\vec{d}$ as the local constants in the variable assignments in $T_{3}$. For the f-block size of core $\left(\right.$ chase $\left.\left(I^{\prime}, \sigma\right)\right)$ the following property can be shown:

Claim 3. The instance core $\left(\operatorname{chase}\left(I^{\prime}, \sigma\right)\right)$ has f-block size $\geq m+1$.

The proof of this claim essentially consists of two steps: First, we observe that the set of facts generated by $T_{1}$ and by $T_{2}$ have labeled nulls of the form $\hat{f}(\vec{a})$ in common, where $\vec{a}$ is a vector of constants used in the variable instantiations at the joint ancestor triggerings of $T_{1}$ and $T_{2}$. This is an immediate consequence of Lemma 3.14. It can then be shown that also the facts generated by $T_{3}$ contain these nulls. In other words, the facts generated by $T_{3}$ in chase $\left(I^{\prime}, \sigma\right)$ are connected to the facts generated by $T_{1}$ and by $T_{2}$. To complete the proof of Claim 3 , it remains to show that $B$ persists in the chase $\left(I^{\prime}, \sigma\right)$ and that at least one more fact is connected to $B$ and also persists in the core. The latter property can be established for the fact $A_{3}$ generated by $T_{3}$, which corresponds to $A_{i}$ in the facts generated by $T_{i}$ for $i \in\{1,2\}$. Since $A_{3}$ contains a null $\hat{f}\left(\vec{a} \cdot \overrightarrow{d^{\prime}}\right)$ with $\overrightarrow{d^{\prime}} \subseteq \vec{d}$, no endomorphism on chase $\left(I^{\prime}, \sigma\right)$ can map it to one of the facts in $B \subseteq \operatorname{chase}\left(I^{\prime}, \sigma\right)$.

It is worth briefly reflecting on the different proof methods applied in Theorem 3.1 (in particular, Lemma 3.22) and in Theorem 4.10 above. Recall that the proofs in Section 3 made use of $k$-patterns, where $k$ depends on the size of the nested tgds involved. In contrast, the proof of Theorem 4.10 above only uses 2-patterns-independently of the size of the nested tgds. In principle, the crucial step in both cases is the extension of a homomorphism: In case of Lemma 3.22, the very essence of the lemma is the existence of an extension of a given homomorphism. In case of Theorem 4.10, the extension of a homomorphism is hidden in the proof of step 3. The important difference between these two proofs is that in case of Lemma 3.22, the extension of the homomorphism has to be constructed yet. In case of Theorem 4.10, we just need to prove certain properties of an assumed extension of the original homomorphism. Obviously, actually constructing the extension of a given homomorphism is more difficult than establishing certain properties of the assumed extension of a given homomorphism. Hence, the former requires stronger tools, namely, $k$-patterns. It will turn out that this is also the case in the proof of Theorem 4.14 below.

But first let us recall the following claim from Reference [7]:

CLAIM 1. There is an algorithm for the following problem: Given an SO tgd $\sigma$ and a positive integer $b$, is the f-block size of $\sigma$ bounded by $b$ ?

Our desired Theorem 4.3 would follow immediately by combining Theorem 4.2 and Theorem 4.10 with Claim 1. Though the algorithm for Claim 1 presented in Reference [7, Theorem 5.2] appears to be correct, the proof of correctness of the algorithm given there has a flaw, which 

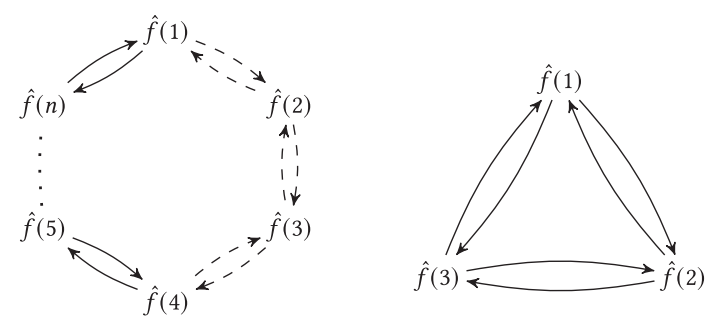

Fig. 8. The undirected cycle of length $n$ on the left side and of length 3 on the right side.

will be pointed out in the sequel. It should be noted that the above claim would also follow from Theorem 3 in Reference [24] together with Theorem 4.10 in Reference [9]; however, Theorem 3 in Reference [24] is stated without proof. In view of this state of affairs, we prove that Claim 1 indeed holds for nested GLAV mappings. For this purpose, we introduce the following concept:

Definition 4.11. A schema mapping $\mathcal{M}$ is said to have a bounded anchor if there exists an integer $a$ such that for every source instance $I$ and for every connected target instance $J$ with $J \subseteq \operatorname{core}(\operatorname{chase}(I, \mathcal{M}))$, there are a source instance $I^{\prime}$ and a connected target instance $J^{\prime}$ such that

- $\left|I^{\prime}\right| \leq a|J|$;

- $\left|J^{\prime}\right| \geq|J|$ and $J^{\prime} \subseteq \operatorname{core}\left(\operatorname{chase}\left(I^{\prime}, \mathcal{M}\right)\right)$.

We say that the bounded anchor of $\mathcal{M}$ is witnessed by $a$.

We extend this notion to classes of schema mappings.

Definition 4.12. Let $C$ be a class of schema mappings. We say that $C$ has effective bounded anchor, if there exists a recursive function $a: C \rightarrow \mathbb{N}$ such that every schema mapping $\mathcal{M}$ in $C$ has bounded anchor witnessed by $a(\mathcal{M})$.

For understanding the intuition behind the concept of bounded anchor, let us consider the following example:

Example 4.13. Let $\sigma$ be the following plain SO tgd:

$$
\exists f \forall x \forall y(S(x, y) \rightarrow R(f(x), f(y)) \wedge R(f(y), f(x))) .
$$

Suppose we want to determine whether $\sigma$ has a bounded anchor. Intuitively, what a bounded anchor gives is the following guarantee: If we find an f-block of a certain size in the target instance, then there is a "small" source instance (the "anchor") that also yields an f-block of at least that certain size in the target instance. Here, "small" means that the source instance is of the same size as the f-block up to a constant factor.

Showing that a schema mapping has bounded anchor is structurally not an easy task, as it involves showing that for any source and connected target instance, we can find such an anchor. All the more, it will be interesting to see that nested GLAV mappings indeed always have bounded anchor. Let us now illustrate, based on our example SO tgds $\sigma$, why it is not even simple to find such an anchor for a given source instance (or rather, class of source instances).

Let $I_{n}=\{S(1,2), S(2,3), \ldots, S(n, 1)\}$ be the source instance consisting of a directed cycle of length $n$ for some $n \geq 3$. Then chase $\left(I_{n}, \sigma\right)$ is the undirected cycle of length $n$. Let $n$ be an odd number. It follows that core $\left(\operatorname{chase}\left(I_{n}, \sigma\right)\right)$ is also the undirected cycle of length $n$, which we depict on the left side of Figure 8 for $n>5$ (each arc, whether it is solid or dashed, denotes an $R$-atom). We now take $J$ to be the subinstance of core $\left(\operatorname{chase}\left(I_{n}, \sigma\right)\right)$ consisting of the dashed edges on the left side of Figure 8 (i.e., six $R$-atoms denoted by the six dashed arcs). Intuitively, the definition of 
bounded anchor requires us to find a "small" source instance $I^{\prime}$ that gives rise to a connected $J^{\prime}$ of size at least $|J|=6$ such that $J^{\prime}$ is contained in core $\left(\operatorname{chase}\left(I^{\prime}, \sigma\right)\right)$. Here, "small" means that the size of $I^{\prime}$ may depend on the size of $J$ but not on $n$. Now observe that no such small source instance can be constructed using the atoms of $I_{n}$ : If $I^{\prime}$ is any proper subinstance of $I_{n}$, then core $\left(\operatorname{chase}\left(I^{\prime}, \sigma\right)\right)$ is just an undirected $R$-edge. However, we can meet the requirements in the definition of bounded anchor by taking $I^{\prime}=I_{3}$ (note that for each $n>3, I_{3} \nsubseteq I_{n}$ holds). Indeed, $\operatorname{core}\left(\operatorname{chase}\left(I_{3}, \sigma\right)\right)$ is the undirected cycle of size 3, depicted on the right side of Figure 8.

Note that Example 4.13 yields a counter-example to a step in the proof of correctness of the algorithm in Theorem 5.2 in Reference [7], where the search for $I^{\prime}$ and $J^{\prime}$ was confined to subsets of the given instances $I$ and $J$.

We show next that the class of nested GLAV mappings indeed has effective bounded anchor.

THEOREM 4.14. The class of nested GLAV mappings has effective bounded anchor.

Proof IdEA. As before, by Lemma 4.5, it suffices to consider a single nested $\operatorname{tgd} \sigma$. We have to show that there exists a recursive function $a$ that, for a given schema mapping specified by a nested $\operatorname{tgd} \sigma$, returns an integer $a(\sigma)$ witnessing bounded anchor for $\sigma$. Let $I$ be a source instance and let $J \subseteq \operatorname{core}(\operatorname{chase}(I, \sigma))$ be connected. We have to show that there exists a source instance $I^{\prime}$ with $\left|I^{\prime}\right| \leq a(\sigma)|J|$ and connected $J^{\prime} \subseteq \operatorname{core}\left(\operatorname{chase}\left(I^{\prime}, \sigma\right)\right)$ with $\left|J^{\prime}\right| \geq|J|$.

The proof reuses the key ideas of the proof of Theorem 3.1: Analogously to Lemma 3.22, let $k=v_{\sigma} w_{\sigma}+1$, and define $a(\sigma)$ as the maximum size of the canonical source instance of any $k$ pattern of $\sigma$. As in Lemma 3.18, we may restrict ourselves to canonical source and target instances, referred to as $I$ and $J$. Moreover, by Lemma 3.17, any pattern $p$ can be obtained from a $k$-pattern $p_{0}$ by a sequence of $k^{+}$-increments, i.e., we get a sequence of patterns $p_{0}, \ldots, p_{n}$ with $p_{n}=p$ and $p_{i-1} \leq_{k} p_{i}$ for every $i$. By Lemma 3.22, we know that if $p_{i} \leq_{k} p$ holds, then certain homomorphisms from $J_{p_{i}}$ can be extended to homomorphisms from $J_{p}$. We use this fact to argue that any homomorphism $h$ from $J_{p_{i}}$ to $\operatorname{core}\left(\operatorname{chase}\left(I_{p_{i}}, \sigma\right)\right)$ preserves the subinstance $J_{p_{i}} \cap J$ of $\operatorname{core}\left(\operatorname{chase}\left(I_{p_{i}}, \sigma\right)\right)$, since, otherwise, the extension of $h$ to a homomorphism from $J$ to $\operatorname{core}(\operatorname{chase}(I, \sigma))$ would also reduce the subinstance $J$ of $\operatorname{core}(\operatorname{chase}(I, \sigma))$.

Having shown that the class of nested GLAV mappings has effective bounded anchor, we can now prove that Claim 1 indeed holds for the class of nested GLAV mappings.

THEOREM 4.15. Let $C$ be a class of schema mappings that has effective bounded anchor. Then the following problem is decidable: Given a schema mapping $\mathcal{M}$ in $C$ and a positive integer $b$, is the f-block size of $\mathcal{M}$ at most $b$ ?

Proof. Let $a$ denote the witness of bounded anchor of $\mathcal{M}$. We test for all source instances $I$ with $|I| \leq a(b+1)$ whether the f-block size of core $(\operatorname{chase}(I, \Sigma))$ is at most $b$. There are finitely many such instances (up to isomorphism) and each test itself is decidable by computing and inspecting the core. If at least one of these tests returns an f-block size greater than $b$, we return that the f-block size is larger than $b$ ("no"). Otherwise, we return that the f-block size is at most $b$ ("yes").

For the correctness, it is clear that in the "no" case, if we witness an f-block of size larger than $b$ for some source instance, then the f-block size is indeed larger than $b$. We thus have to show that in the "yes" case, if for all source instances $K$ with $|K| \leq a(b+1)$ the f-block size of core $(\operatorname{chase}(K, \Sigma))$ is at most $b$, then also for all other, larger source instances the f-block size is at most $b$. We will prove this by showing the following: If for source instance $I$ with $|I|>a(b+1)$ we have that core $(\operatorname{chase}(I, \Sigma))$ has f-block size at least $b+1$, then there is also some source instance $I^{\prime}$ with $\left|I^{\prime}\right| \leq a(b+1)$ s.t. $\operatorname{core}\left(\operatorname{chase}\left(I^{\prime}, \Sigma\right)\right)$ has f-block size at least $b+1$.

Assume that core $(\operatorname{chase}(I, \mathcal{M}))$ contains an f-block $B$ of size at least $b+1$. We take an arbitrary connected $J \subseteq B$ with $|J|=b+1$. By assumption, $J$ is connected. Since $\Sigma$ has bounded anchor 
witnessed by $a$, we know that there is $I^{\prime}$ with $\left|I^{\prime}\right| \leq a|J|$ and connected $J^{\prime} \subseteq \operatorname{core}\left(\operatorname{chase}\left(I^{\prime}, \mathcal{M}\right)\right)$ with $\left|J^{\prime}\right| \geq|J|$. Now, since $|J|=b+1$, we know that $\left|I^{\prime}\right| \leq a|J|=a(b+1)$ satisfies the requirement on the size of $I^{\prime}$. Since $J^{\prime}$ is connected, and $\left|J^{\prime}\right| \geq|J|=b+1$, we also produce the required size of the f-block.

Now, by exploiting the fact that nested GLAV mappings have both effective threshold for f-block size and effective bounded anchor, we show that there is an algorithm for deciding whether the f-block size of a nested GLAV mapping is bounded.

THEOREM 4.16. Let $C$ be a class of schema mappings having both effective threshold for f-block size and effective bounded anchor. Then the following problem is decidable: Given a schema mapping $\mathcal{M}$ in $\mathcal{C}$, does $\mathcal{M}$ have bounded f-block size?

Proof. Let $f$ be the recursive function providing the effective threshold for f-block size for schema mappings in $C$. Consider the following algorithm: Given a mapping $\mathcal{M}$ in $C$, compute the bound $b=f(\mathcal{M})$ for the effective threshold for f-block size. Since $C$ has effective bounded anchor, we can use the algorithm in Theorem 4.15 to test whether $\mathcal{M}$ has f-block size bounded by $b$. If it does, return that $\mathcal{M}$ has bounded f-block size; otherwise, return that $\mathcal{M}$ has unbounded f-block size.

By assembling all the preceding machinery, we can now prove the main result of this section.

Proof of Theorem 4.3. By Theorem 4.10 and Theorem 4.14, the class of nested GLAV mappings has both effective threshold for f-block size and effective bounded anchor. Therefore, by Theorem 4.16, the following problem is decidable: Given a nested GLAV mapping, does it have bounded f-block size? Thus, together with Theorem 4.2, we get the decidability result stated in Theorem 4.3.

\subsection{Plain SO Tgds vs. Nested GLAV Mappings}

We have just seen that there is an algorithm to differentiate between nested GLAV mappings and GLAV mappings. It is not known, however, whether there is an algorithm to differentiate between plain SO tgds and nested GLAV mappings. In other words, it is not known whether or not the following problem is decidable: Given a plain SO $\operatorname{tgd} \sigma$, is there a nested GLAV mapping $\mathcal{M}$ such that $\sigma$ is logically equivalent to $\mathcal{M}$ ?

What tools are there for showing that a particular plain SO $\operatorname{tgd} \sigma$ is not logically equivalent to any nested GLAV mapping? Since plain SO tgds are expressible in second-order logic while nested GLAV mappings are expressible in first-order logic, it suffices to show that $\sigma$ is not firstorder expressible. The standard methods for doing this are Ehrenfeucht-Fraïssé games or locality methods (see Reference [23]). In fact, this method is behind the proof in Reference [2] that the plain SO tgd

$$
\exists f \forall x \forall y(S(x, y) \rightarrow R(f(x), f(y)))
$$

is not logically equivalent to any nested GLAV mapping. In what follows, we take a totally different approach and give two different sufficient conditions for showing that a given SO tgd is not logically equivalent to any nested GLAV mapping. The idea behind these conditions is as follows: Suppose we suspect that a given plain SO $\operatorname{tgd} \sigma$ is not logically equivalent to any nested GLAV mapping. In this case, $\sigma$ is not equivalent to any GLAV mapping either and, hence, $\sigma$ has unbounded f-block size by Theorem 4.2. Now, a schema mapping may have unbounded f-block size for a number of different reasons. However, we will show that a nested GLAV mapping can have unbounded f-block size only for certain specific reasons that are not shared by all plain SO tgds. Therefore, if the given SO $\operatorname{tgd} \sigma$ is contained in one of these categories, then we can conclude that indeed $\sigma$ is not logically equivalent to any nested GLAV mapping. 
F-Degree. Before stating the first result of this section, we need to relativize the notion of bounded f-block size to a class of source instances. Assume that $\mathcal{M}$ is a schema mapping specified by an SO $\operatorname{tgd}$ and $C$ is a class of source instances. We say that $\mathcal{M}$ has bounded f-block size on $C$ if there is a positive integer $b$ such that for every source instance $I$ in $C$, the f-block size of $\operatorname{core}(\operatorname{chase}(I, \mathcal{M}))$ is at most $b$; otherwise, we say that $\mathcal{M}$ has unbounded f-block size on $C$. Clearly, $\mathcal{M}$ has bounded f-block size if it has bounded f-block size on the class of all source instances.

If $G$ is an undirected graph and $v$ is a node of $G$, then the degree of $v$ is the number of edges incident to $v$. The degree of $G$ is the maximum degree of its nodes. We say that a schema mapping has bounded $f$-degree on $C$ if there is a positive integer $d$ such that for every source instance $I$ in $C$, the degree of every f-block of $\operatorname{core}(\operatorname{chase}(I, \mathcal{M}))$ is at most $d$; otherwise, we say that $\mathcal{M}$ has unbounded $f$-degree on $C$.

Before illustrating the close connection between f-block size and f-degree for nested GLAV mappings, we show that there is no such close connection between f-block size and f-degree for plain SO tgds. This simple proof for SO tgds allows the reader to get an intuition of the two concepts before the more complex construction for nested GLAV mappings.

Proposition 4.17. There is a plain SO tgd $\tau$ and a class $C$ of source instances such that $\tau$ has unbounded f-block size on $C$, but bounded f-degree on $C$.

Proof. Let $\tau$ be the plain SO $\operatorname{tgd} \exists f \forall x \forall y(S(x, y) \rightarrow R(f(x), f(y)))$ and let $C$ be the class of all source instances $I$ such that $S$ is a successor relation. If $I \in C$, then core $(\operatorname{chase}(I, \tau))$ consists of a single f-block of the same size as $S$ in which no null occurs more than twice. Thus, the f-block size of $\tau$ on $C$ is unbounded, but the f-degree of $\tau$ on $C$ is 2 .

We now show the close connection between f-degree and f-block size for nested GLAV mappings. From this it will immediately follow that the plain SO tgd $\tau$ in Proposition 4.17 is not logically equivalent to any nested GLAV mapping.

Theorem 4.18. Let $\mathcal{M}$ be a nested GLAV mapping and $C$ a class of source instances. Then $\mathcal{M}$ has bounded f-block size on $C$ if and only if $\mathcal{M}$ has bounded $f$-degree on $C$.

The theorem follows immediately from the following lemma. Note that the lemma holds even if we consider the Gaifman graph of facts for arbitrary instancesnot just for the core of some chase result. But for our purposes it will suffice to prove this restricted version.

Lemma 4.19. Let $\mathcal{M}$ be a nested GLAV mapping. The following claims hold:

(1) For an arbitrary source instance $I$, it holds that core $($ chase $(I, \mathcal{M}))$ has f-block size greater than f-degree.

(2) For each integer $k$, there exists an integer $m_{k}$, such that for an arbitrary source instance I, if core $(\operatorname{chase}(I, \mathcal{M}))$ has f-block size $b \geq m_{k}$, then it has $f$-degree $g \geq k$.

Proof. (1) Since the f-degree $g$ of core $(\operatorname{chase}(I, \mathcal{M}))$ is the maximum number of facts sharing at least one null with a given fact $R(\vec{c})$, the f-block size of core $(\operatorname{chase}(I, \mathcal{M}))$ is at least $g+1$.

(2) We start by showing the following claim:

ClAIm A. For each integer $n$, there exists an integer $m_{n}$ such that for an arbitrary source instance $I$, if core $(\operatorname{chase}(I, \mathcal{M}))$ has f-block size $b \geq m_{n}$, then at least one null occurs at least $n$ times in $\operatorname{core}(\operatorname{chase}(I, \mathcal{M}))$.

Proof of Claim A. Let $n$ be an arbitrary integer. We have to show that there exists an integer $m_{n}$ such that for an arbitrary source instance $I$, if core $(\operatorname{chase}(I, \mathcal{M}))$ has f-block size $b \geq m_{n}$, then there are at least $n$ occurrences of some null in $\operatorname{core}(\operatorname{chase}(I, \mathcal{M}))$. 
Let $w$ denote the maximum conclusion size (i.e., number of atoms in the conclusion) of a part of a nested $\operatorname{tgd}$ of $\mathcal{M}$. Let $p$ denote the maximum number of parts in a nested tgd of $\mathcal{M}$. Let $d$ denote the maximum nesting depth of a nested tgd of $\mathcal{M}$. Let $e$ denote the maximum number of existentially quantified variables in a part of a nested $\operatorname{tgd}$ of $\mathcal{M}$. Define $m_{n}=w(p \ell)^{d}$ where $\ell=n d e$. In the rest of the proof of this claim, we will show why this choice of $m_{n}$ yields our desired result.

Let $I$ be an arbitrary source instance and assume that core $(\operatorname{chase}(I, \mathcal{M}))$ has f-block size $b \geq m_{n}$. Let $J_{B}$ be an arbitrary f-block of core $(\operatorname{chase}(I, \mathcal{M}))$ of size $b \geq m_{n}$. Let $T$ be the RC-chase tree w.r.t. $J_{B}$. Since $J_{B}$ has size at least $m_{n}=w(p \ell)^{d}$, and we know that a single triggering can produce at most $w$ facts, we know that there must be at least $\left\lceil m_{n} / w\right\rceil=(p \ell)^{d}$ triggerings in $T$. Since the height of $T$ is bounded by $d$, we know that some triggering in $T$ must have at least $p \ell$ child triggerings. Note that by definition, the child triggerings of a triggering in a chase tree have the same input assignment $\vec{a}_{0}$. Of these $p \ell$ triggerings, since there are at most $p$ parts of nested tgds in $\mathcal{M}$, one of these parts $\sigma_{j}$ must have at least $\ell$ triggerings $t_{1}, \ldots, t_{\ell}$.

Let $J_{i}=\left\{f a c t s(t) \mid t \in \operatorname{desc}\left(t_{i}\right)\right\}$ denote the subinstance of $J_{B}$ generated by the subtree $\operatorname{desc}\left(t_{i}\right)$ rooted at $t_{i}$. Note that all of these $J_{i}$ are non-empty by the definition of $T$. From Lemma 3.14, we can conclude that $J_{1} \cup \ldots \cup J_{\ell}$ can belong to the same f-block only if each of the facts in $J_{1} \cup \ldots \cup J_{\ell}$ contains a null $\hat{f}(\vec{c})$ with $f$ from $\operatorname{anc}\left(\sigma_{j}\right)$ and $\vec{c}$ a prefix of $\vec{a}_{0}$. Observe that there are at most $d e$ Skolem functions $f$ (as $e$ denotes the maximum number of existentially quantified variables in a part of a nested tgd in $\mathcal{M}$ and $d$ bounds the depth)-and thus at most $d e$ nulls instantiating the Skolem terms in $J_{1} \cup \ldots \cup J_{\ell}$ (since their arguments $\vec{c}$ are a prefix of $\vec{a}_{0}$ ). Observe that there are at least $n d e$ facts in $J_{1} \cup \ldots \cup J_{\ell}$ (as $\ell=n d e$ and all $J_{i}$ are non-empty). Thus, there must exist at least one null that occurs at least $n d e / d e=n$ times, which was to be shown.

It is not difficult to see that Claim A implies the second claim of the lemma. Indeed, let $k$ be an arbitrary integer. We have to show that there exists an integer $m_{k}$, such that for an arbitrary source instance $I$, if $\operatorname{core}(\operatorname{chase}(I, \mathcal{M}))$ has f-block size $b \geq m_{k}$, then it has f-degree $g \geq k$. Let $r$ denote the maximum arity of a relation in the target schema of $\mathcal{M}$. We now invoke Claim A with $n=$ $r(k+1)$, yielding an integer $m_{n}$ such that for an arbitrary source instance $I$, if core $($ chase $(I, \mathcal{M}))$ has f-block size $b \geq m_{n}$, then at least one null occurs at least $n$ times in $\operatorname{core}(\operatorname{chase}(I, \mathcal{M}))$. Define $m_{k}=m_{n}$. Let $I$ be an arbitrary source instance such that core $($ chase $(I, \mathcal{M}))$ has f-block size $b \geq m_{k}$ (and, equivalently, $b \geq m_{n}$ ). We thus know that there are at least $n$ occurrences of some null in $\operatorname{core}(\operatorname{chase}(I, \Sigma))$.

Since there are $n=r(k+1)$ occurrences of one null in a block of core $(\operatorname{chase}(I, \mathcal{M}))$, there are at least $\lceil n / r\rceil=k+1$ facts in this block (as $r$ is the maximum arity of a relation in the target schema). Moreover, these facts give rise to a clique of the same size $\lceil n / r\rceil$ in the Gaifman graph of facts of $\operatorname{core}(\operatorname{chase}(I, \mathcal{M}))$, and thus the $\mathrm{f}$-degree $g$ of $\operatorname{core}(\operatorname{chase}(I, \mathcal{M}))$ is at least $\lceil n / r\rceil-1=k$. That is, it holds that $\mathrm{f}$-degree $g \geq k$ as desired in the second claim of the lemma, which was to be shown.

Informally, the preceding theorem asserts that nested tgds can achieve unbounded f-block size on a class of source instances only because some null value appears unboundedly often in the core of the universal solutions of such instances. In contrast, plain SO tgds can achieve unbounded f-block size in more complex ways, as evidenced by Proposition 4.17.

Altogether, f-degree is an easy-to-use tool for showing that a schema mapping is not logically equivalent to a nested GLAV mapping (as we did in Proposition 4.17). However, it is not always sufficient for dealing with more complex schema mappings, as the next example shows.

Example 4.20. Consider the following plain SO $\operatorname{tgd} \sigma$ :

$$
\exists f \exists g \forall x \forall y \forall z(S(x, y) \wedge Q(z) \rightarrow R(f(z, x), f(z, y), g(z))) .
$$




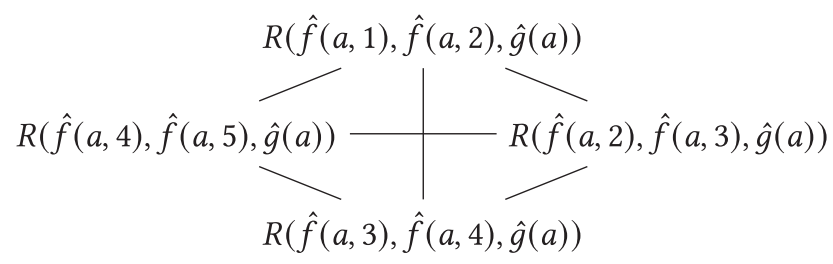

Fig. 9. Gaifman graph of facts of Example 4.20 for a successor relation of length 5.

It will turn out that $\sigma$ is not logically equivalent to any nested GLAV mapping. However, it is easy to see that each $\mathrm{f}$-block is a clique, which implies that for every class $C$ of source instances, $\sigma$ has unbounded f-block size on $C$ if and only if it has unbounded $\mathrm{f}$-degree on $C$. For example, if $C$ is the class of source instances in which $S$ is a successor relation and $Q$ is a singleton, then each f-block is a clique of the form depicted in Figure 9. Thus, Theorem 4.18 cannot be used to show that $\sigma$ is not logically equivalent to any nested GLAV mapping.

The preceding example shows that, in addition to Theorem 4.18, a different structural tool is needed to differentiate between plain SO tgds and nested GLAV mappings. To appreciate how delicate this differentiation can be, we note that a plain SO tgd and a nested tgd may have the same f-blocks (up to isomorphism) on some classes of instances, yet the plain SO tgd is not logically equivalent to any nested GLAV mapping.

Example 4.21. Consider the following plain SO $\operatorname{tgd} \sigma^{\prime}$ :

$$
\exists f \exists g \forall x \forall y \forall z(S(x, y) \wedge Q(z) \rightarrow R(f(z, x, y), g(z), x)) .
$$

This dependency is logically equivalent to the following nested tgd:

$$
\forall z(Q(z) \rightarrow \exists u(\forall x \forall y(S(x, y) \rightarrow \exists v R(v, u, x)))) .
$$

For the source instances in which $S$ is a successor relation, the f-blocks are isomorphic to those of the plain SO tgd $\sigma$ in Example 4.20, i.e., they are complete graphs (see Figures 9 and 11). Yet, as we are about to discover, $\sigma$ is not logically equivalent to any nested GLAV mapping. Intuitively, the reason is that $\sigma$ can "build arbitrarily long chains of nulls," while $\sigma^{\prime}$ can not, but we shall now introduce a concrete tool that allows one to make this distinction on a formal level.

Path Length. To cope with this situation, we need to look beyond the Gaifman graph of facts. Recall that the Gaifman graph of facts is the graph whose nodes are the facts and there is an edge between two facts if they share a null. Let $J$ be a target instance. The Gaifman graph of nulls of $J$ is the graph whose nodes are the nulls of $J$, and there is an edge between two nulls if they occur in the same fact in $J$.

It turns out that properties of the Gaifman graph of nulls can be used to show inexpressibility in situations where the structure of the Gaifman graph of facts is of no help. More formally, the path length of an undirected graph $G$ is the length of the longest simple path in $G$, where a simple path is a path that visits each node in $G$ at most once. We say that a schema mapping $\mathcal{M}$ specified by an SO tgd has bounded path length if there is a positive integer $l$ such that for every source instance $I$, the path length of the Gaifman graph of nulls of $\operatorname{core}(\operatorname{chase}(I, \mathcal{M}))$ is at most $l$; otherwise, we say that $\mathcal{M}$ has unbounded path length.

Theorem 4.22. Every nested GLAV mapping has bounded path length.

Proof. Let $\mathcal{M}$ be a nested GLAV mapping. Let $I$ be an arbitrary source instance. We show that the length of the longest simple path in the Gaifman graph of nulls of core $(\operatorname{chase}(I, \mathcal{M}))$ is bounded by a constant only depending on $\mathcal{M}$. Let $y_{1}, \ldots, y_{n}$ be an arbitrary simple path in the 


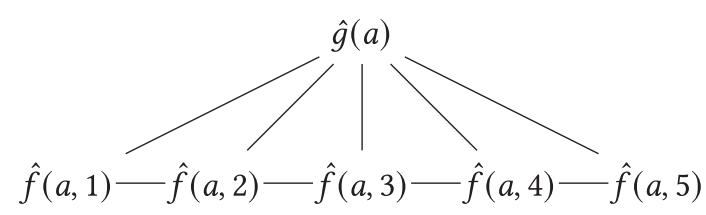

Fig. 10. Gaifman graph of nulls of Example 4.20 for a successor relation of length 5.

Gaifman graph of nulls of core $(\operatorname{chase}(I, \mathcal{M}))$. We denote by $e_{i}$ the edge $\left(y_{i-1}, y_{i}\right)$ of this path and by $A_{i}$ the atom in core $(\operatorname{chase}(I, \mathcal{M}))$ where $y_{i-1}$ and $y_{i}$ occur together (if there is more than one such atom, we choose an arbitrary one).

Let $\mathcal{A}=A_{1} \cup \ldots \cup A_{n}$ and let $T$ be the RC-chase tree w.r.t. $\mathcal{A}$. Let $d$ be the maximum depth of a nested tgd of $\mathcal{M}$. Let $e$ be the maximum number of existentially quantified variables in a part of a nested $\operatorname{tgd}$ of $\mathcal{M}$.

Claim 1. In $T$, every node has at most $m=d e+1$ child nodes.

Proof of Claim 1. Consider an arbitrary node $t$ in $T$. Suppose that $t$ has $s$ child nodes $t_{1}, \ldots, t_{s}$. By construction of $T$, the subtree rooted at each $t_{\alpha}$ contains at least one atom $A_{i_{\alpha}} \in \mathcal{A}$. If there are several such atoms of $\mathcal{A}$ in the subtree rooted at node $t_{\alpha}$, then we choose $A_{i_{\alpha}}$ arbitrarily. W.l.o.g., assume that the nodes $t_{1}, \ldots, t_{s}$ are arranged in such an order that $i_{1}<i_{2}<\cdots<i_{s}$.

Let $\alpha \in\{1, \ldots, s-1\}$ be arbitrary. Consider $t_{\alpha}$ and $t_{\alpha+1}$ : There must exist atoms $A_{j}$ and $A_{j+1}$ with $i_{\alpha} \leq j<j+1 \leq i_{\alpha+1}$ such that $A_{j}$ is in the subtree rooted at $t_{\alpha}$ and $A_{j+1}$ is outside this subtree. By definition of the atoms $A_{i}$ (i.e., the fact that they realize the edges of the path $y_{1}, \ldots, y_{n}$ in the Gaifman graph of nulls), $A_{j}$ and $A_{j+1}$ contain the null $y_{j}$. Since $A_{j}$ is in the subtree below $t_{\alpha}$ and $A_{j+1}$ is outside this subtree, $y_{j}$ must be one of the nulls introduced in the branch from the root to $t$, i.e., $y_{j}$ corresponds to a null $\hat{f}(\vec{c})$ where $\vec{c}$ is a prefix of the input assignment $\vec{a}_{0}$ of $t_{\alpha}$ (which is the same as the input assignment of $t_{\alpha+1}$, as sibling triggerings by definition have the same input assignments).

Recall that we are considering a simple path $y_{1}, \ldots, y_{n}$. Hence, the nulls $y_{j}$ that exist for each $\alpha$ must be pairwise-distinct for different values of $\alpha$. The number of nulls $y_{j}$ (which, as discussed before, are of the form $\hat{f}(\vec{c})$ where $\vec{c}$ is a prefix of the input assignment $\vec{a}_{0}$ shared by all $t_{\alpha}$ ) is bounded by $d e$. Hence, the number $s$ of child nodes $t_{\alpha}$ of $t$ must be bounded by $m=d e+1$. $\triangleleft$

By Claim 1, it holds that $T$ has at most $m^{d}$ nodes. Let $w$ be the maximum conclusion size, i.e., the maximum number of atoms in the conclusion of a part of a nested tgd of $\mathcal{M}$. Then $\mathcal{A}$ contains at most $m^{d} w$ atoms. Let $r$ be the maximum arity of the relation symbols in the target schema. Thus, since each atom can contain at most $r$ nulls, and all nulls $y_{1}, \ldots, y_{n}$ are contained in the atoms $\mathcal{A}=\left\{A_{1}, \ldots, A_{n}\right\}$, we get the upper bound $m^{d} w r$ on the number $n$ of nulls.

Equipped with Theorem 4.22, we now have a tool to show that the plain SO $\operatorname{tgd} \sigma$ of Example 4.20 is not logically equivalent to any nested GLAV mapping. This is so because $\sigma$ has unbounded path length, which can be checked using successor relations in $S$ as source instances (see Figure 10, where the Gaifman graph of nulls contains a simple path of length 4, in contrast to Figure 12).

\section{ADDING SOURCE CONSTRAINTS}

In the previous section, we showed that it is decidable whether a schema mapping specified by nested tgds is equivalent to a GLAV mapping. The decidability of whether an SO tgd is equivalent to a GLAV mapping is still open. In this section, we give evidence that the problem may indeed be harder for SO tgds: It is undecidable whether a plain SO tgd is equivalent to a GLAV mapping in the presence of a single source key dependency. In contrast, we will show that for nested tgds and 


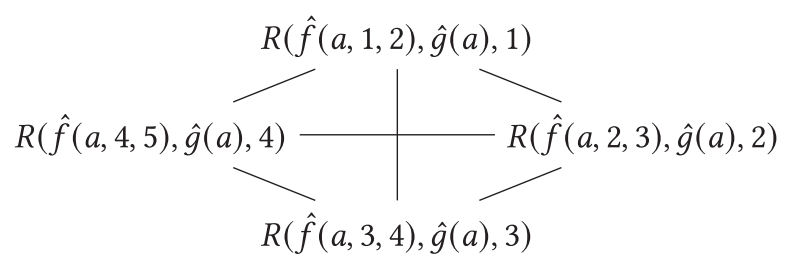

Fig. 11. Gaifman graph of facts of Example 4.21 for a successor relation of length 5.

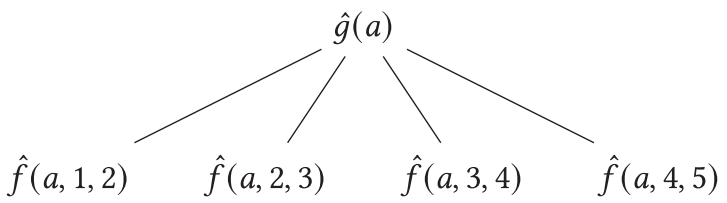

Fig. 12. Gaifman graph of nulls of Example 4.21 for a successor relation of length 5.

in the presence of arbitrary source egds, equivalence to GLAV is still decidable. Completing the picture, we also show that the implication problem of nested tgds discussed in Section 3 remains decidable in the presence of source egds.

Recall that Theorem 4.2 reduces the problem of whether a plain SO tgd is equivalent to a GLAV mapping to the problem of deciding whether it has bounded f-block size. This theorem, which is derived from Proposition 3.14 and Theorem 4.10 in Reference [9], thus played an important role in Section 4. A close inspection of the proofs of Proposition 3.14 and Theorem 4.10 in Reference [9] shows that these results (and therefore Theorem 4.2) still hold in the presence of source egds. This fact is used below.

THEOREM 5.1. It is undecidable whether a given plain SO tgd is equivalent to a GLAV mapping in the presence of a single source key dependency.

Proof. By the above comments it suffices to show the undecidability of the following problem: Given a schema mapping specified by a plain SO tgd and a source key dependency, does the mapping have bounded f-block size? Our proof is by reduction from the halting problem.

Thus, for a given Turing machine, we construct an SO tgd that "simulates" the computation of the Turing machine. We first describe the basic structure of our proof and then give the construction. The basic structure is to represent a run of a Turing machine (state and tape configurations) together with a successor relation in the source instance. We construct a key dependency to ensure that in the supposed successor relation, each element has a unique predecessor. The SO tgd then guarantees that the f-block size is bounded if and only if the Turing machine halts.

The particular challenge of this reduction is how to handle incorrect and missing information in the source instance. For incorrect information, we define "guards" that lead to a collapse of f-block size. The more problematic part is missing information, for which we define a specific onedimensional enumeration of the two-dimensional (time and tape space) structure of the Turing machine's run in the target. When we reach a certain point of this enumeration, we know that no essential information is missing up to that point.

The final challenge is how to handle the effects of unintended structure of the successor relation given in the source instance. While the single key dependency gives us some control over the structure, we define "traps" that address the effects of deviating from the successor relation in the target in ways not handled by that single key dependency. 
Construction. We now show the reduction, and we give the correctness proof in the Appendix. Let $\mathrm{TM}$ be a Turing machine. We now define a schema mapping from a source schema to a target schema specified by an SO $\operatorname{tgd} \sigma$. We assume w.l.o.g. that the Turing machine starts from the empty tape, and that from the halting state, there are no further transitions possible.

Source Schema. For every alphabet symbol $a_{i}$ of the Turing machine, there is a unary predicate $A_{i}$ in the source schema. The intended meaning is that if $A_{i}(x)$ holds, then $x$ equals $a_{i}$. Furthermore, we assume that $a_{0}=\triangleright$ (i.e., the special symbol always marking the left end of the tape) and $a_{1}=\sqcup$ (i.e., the blank symbol). Similarly, for every state $q_{i}$ there is a unary predicate $Q_{i}$ in the source schema, where $q_{0}$ denotes the starting state and $q_{1}$ the halting state. Also, we will use the unary predicate $C_{1}$ to represent a cursor being present, and $C_{0}$ for the cursor being absent.

For describing time and space of the Turing machine computation, we add a unary predicate $Z$ ("zero") as well as a binary predicate $S$ ("successor") to the source schema. The intended meaning is for $Z$ to be the root of a linear order, i.e., $Z(0)$, and for $S$ to describe the order itself, i.e., $S(0,1), S(1,2)$, and so on. For describing the configurations of the Turing machine, we use predicates state and tape. The intended meaning is that state $(x, q)$ denotes that at time $x$, the machine is in state $q$. Similarly, tape $(x, y, a, c)$ denotes that at time $x$ and tape position $y$, the symbol $a$ is stored and the cursor is at this position $(c=1)$ or not $(c=0)$.

Target Schema. The target schema contains a unary predicate $O$ ("origin") and a binary predicate $N$ ("next"). The intended meaning is to represent a linear order, rooted at $O$ and the successor relation stored in $N$. That is, $O$ and $N$ are comparable to $Z$ and $S$ of the source schema. However, the target schema is intended to represent a one-dimensional enumeration of the two-dimensional space-time structure of the Turing machine computation. We will illustrate this idea in detail later.

Dependency. We will now construct the SO $\operatorname{tgd} \sigma$ by describing all parts of the tgd (where all individual symbols are considered to be universally quantified). The final SO tgd is obtained by constructing a conjunction of those parts and adding existential function quantifiers for all function symbols. We first define the abbreviation fail, which, when put on the right-hand side of an SO tgd part and triggered, will lead to the core "collapsing."

- fail : $=O(x) \wedge N(x, x)$

Chain Guards. We require that the start of our linear order described by $Z$ has no predecessor and thus add the following part to our SO tgd:

- $Z(y) \wedge S(x, y) \rightarrow$ fail.

We also require $S$ to be irreflexive:

- $S(x, x) \rightarrow$ fail.

Note that we do not directly enforce functionality on $S$.

Uniqueness Guards. We need to make sure that for every time instant $x$ there is only one distinct state stored. Also, for every time instant $x$ and tape position $y$, we require that only one distinct tape symbol and cursor information is stored. For each $i \neq j$, we thus add the following parts:

- $\operatorname{state}\left(x, q_{i}\right) \wedge \operatorname{state}\left(x, q_{j}\right) \wedge Q_{i}\left(q_{i}\right) \wedge Q_{j}\left(q_{j}\right) \rightarrow$ fail,

- $\operatorname{tape}\left(x, y, a_{i},{ }_{-}\right) \wedge \operatorname{tape}\left(x, y, a_{j},{ }_{-}\right) \wedge A_{i}\left(a_{i}\right) \wedge A_{j}\left(a_{j}\right) \rightarrow$ fail,

- $\operatorname{tape}\left(x, y, \ldots, c_{i}\right) \wedge \operatorname{tape}\left(x, y,{ }_{-}, c_{j}\right) \wedge C_{i}\left(c_{i}\right) \wedge C_{j}\left(c_{j}\right) \rightarrow$ fail. 
Transition Guards. The symbol stored at a specific tape position $y$ and time instant $x$, as well as the fact whether the cursor is located at this tape cell, depends only on the previous time instant $x^{\prime}$, and there only on tape position $y$, the tape position on the left $y_{l}$, and the one on the right $y_{r}$.

We now need to check whether the configuration at time instant $x$ and tape position $y$ is correct according to the definition of the Turing machine. We thus collect all required information for current state $q_{\alpha}$, tape symbol $a_{\beta}$, and cursor information $c_{\beta}$ (1). That is, the previous state $q_{\alpha^{\prime}}$ as well as tape symbol and cursor information at the middle (2), left (3), and right (4) positions in the previous configuration $\left(a_{\beta}\right.$ and $c_{\beta}$ for $\left.p \in\{l, m, r\}\right)$. For such a setting $\pi=\left(q_{\alpha}, q_{\alpha^{\prime}}, a_{\beta}, a_{\beta^{l}}, a_{\beta^{m}}, a_{\beta^{r}}, c_{\beta}, c_{\beta^{l}}, c_{\beta^{m}}, c_{\beta^{r}}\right)$, we can thus define the following abbreviation $\operatorname{check}_{\pi}[x, y]:$

$$
\begin{aligned}
& \operatorname{check}_{\pi}[x, y]:= \\
& \operatorname{state}\left(x, q_{\alpha}\right) \wedge Q_{\alpha}\left(q_{\alpha}\right) \wedge \operatorname{tape}\left(x, y, a_{\beta}, c_{\beta}\right) \wedge A_{\beta}\left(a_{\beta}\right) \wedge C_{\beta}\left(c_{\beta}\right) \\
& \wedge S\left(x^{\prime}, x\right) \wedge \operatorname{state}\left(x^{\prime}, q_{\alpha^{\prime}}\right) \wedge Q_{\alpha^{\prime}}\left(q_{\alpha^{\prime}}\right) \wedge \operatorname{tape}\left(x^{\prime}, y, a_{\beta^{m}}, c_{\beta^{m}}\right) \wedge A_{\beta^{m}}\left(a_{\beta^{m}}\right) \wedge C_{\beta^{m}}\left(c_{\beta^{m}}\right) \\
& \wedge S\left(y_{l}, y\right) \wedge \operatorname{tape}\left(x^{\prime}, y_{l}, a_{\beta^{l}}, c_{\beta^{l}}\right) \wedge A_{\beta_{l}}\left(a_{\beta^{l}}\right) \wedge C_{\beta}\left(c_{\beta^{l}}\right) \\
& \wedge S\left(y, y_{r}\right) \wedge \operatorname{tape}\left(x^{\prime}, y_{r}, a_{\beta^{r}}, c_{\beta^{r}}\right) \wedge A_{\beta_{r}}\left(a_{\beta^{r}}\right) \wedge C_{\beta}\left(c_{\beta^{r}}\right) .
\end{aligned}
$$

For an arbitrary setting $\pi$, we can clearly identify whether or not it correctly follows the description of the Turing machine. We thus build the two sets $\Pi_{\text {good }}$ as the set of all $\pi$ that follow the definition of TM, and $\Pi_{\mathrm{bad}}$ for those that do not. For later use, we also define the special setting $\star=\left({ }_{-},,_{-},,_{-},,_{-},{ }_{-},{ }_{-}\right)$containing anonymous variables instead of constants. The intuition behind this setting is that it only checks for the existence of the relevant tuples and not in addition checks for good or bad ones.

For instance, when in a setting $\pi \in \Pi_{\text {good }}$ all $c_{\beta}$ cursor markers have the value 0 , then this setting corresponds to an inertia rule (i.e., a rule that enforces that, unless changed, the configuration stays the same). When at least one of $c_{\beta^{l}}, c_{\beta^{m}}, c_{\beta^{r}}$ has the value 1 , then this represents a transition rule. A clear example of a $\pi \in \Pi_{\text {bad }}$ is when $c_{\beta^{l}}, c_{\beta^{m}}, c_{\beta^{r}}$ all have the value 0 and $c_{\beta}$ has the value 1 . Note that these sets $\Pi_{\text {good }}$ and $\Pi_{\text {bad }}$ are clearly finite for a fixed Turing machine.

Now for all $\pi_{b a d} \in \Pi_{\text {bad }}$, we add the part:

- $\operatorname{check}_{\pi_{b a d}}[x, y] \rightarrow$ fail.

Note that for the border cases of the two-dimensional configuration structure (initial time instant, first tape position, the diagonal after which only blank symbols are present) adaptations need to be made to $\operatorname{check}_{\pi}[x, y]$. They are technical but do not provide deep insight. In particular, (2) needs to be omitted in the initial time instant, (3) needs to be omitted at the first tape position, and (4) needs to be omitted at the diagonal.

Constructing the Target. Our intention is for the target instance to represent an enumeration of the configuration matrix of the Turing machine. The structure of this enumeration is illustrated in Figure 13. The vertical axis represents time (starting at the top) and the horizontal axis the tape (starting at the left). Note that it is only necessary to represent this triangular part of the configuration matrix, as a Turing machine can in, e.g., 4 steps in time at most reach the 4 th tape cell. To realize this enumeration, we first need to materialize the origin $O$ (corresponding to the black square in Figure 13):

- $Z(x) \rightarrow O(f(x, x))$.

We also need to realize the arcs of the enumeration as $N$ atoms. There are two possible cases. The $\leftarrow$ step goes from the given tape position to the previous one at the same time instant. The $\searrow$ 


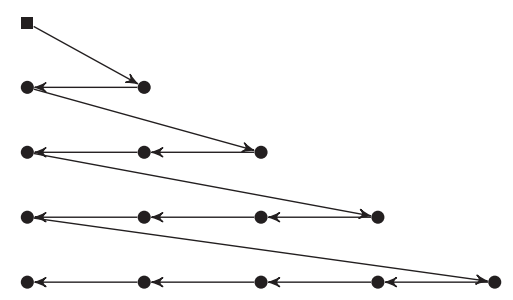

Fig. 13. Intended enumeration represented in the target instance. Arrows denote $N$ atoms. The origin node represented by $O$ is marked by a rectangle.

step to the diagonal in the configuration matrix and the next time instant is made when the initial tape position is reached. These are implemented as follows as SO tgd parts:

- $\operatorname{check}_{\star}[x, y] \wedge S\left(y, y^{\prime}\right) \rightarrow N\left(f\left(x, y^{\prime}\right), f(x, y)\right)$

- $\operatorname{check}_{\star}\left[x^{\prime}, x^{\prime}\right] \wedge S\left(x, x^{\prime}\right) \wedge Z(y) \rightarrow N\left(f(x, y), f\left(x^{\prime}, x^{\prime}\right)\right)$

$(\leftarrow$ transition $)$,

$(\searrow$ transition).

Note that the conditions in the antecedents of the above implications are mutually exclusive, since for any $y$ for which $Z(y)$ holds, there cannot be a predecessor $S\left({ }_{-}, y\right)$.

The key fact about this enumeration is that it uses the successor relation, both in space and in time, only in one direction (the "backwards" direction). This is necessary, because with a single key dependency, we can only guarantee that one direction allows correct navigation (in our case, we will guarantee unique predecessors). The only other navigation step we can ensure to be correct is "jumping to the diagonal" (the time and space index coincide), as illustrated by the diagonal arrows in Figure 13.

Unfoundedness Traps. The idea of the following parts is to get rid of "unfounded" paths, that is, paths not beginning at an origin $O$. For each combination of time instant $x$ and tape position $y$, we add a self-loop in $N$ using the function symbol $h$ and then connect this self-loop to the corresponding function symbol $f$. The effect is that in the core, paths that do not start at an $O$ atom can be "shrunk" into this self-loop, i.e., the core will only contain our self-loop and the connection, but not an unfounded path of arbitrary length. We add:

- $\operatorname{check}_{\star}[x, y] \rightarrow N(h(x, y), h(x, y))$,

- $\operatorname{check}_{\star}[x, y] \rightarrow N(h(x, y), f(x, y))$.

Key Dependency. We now make sure that in the $S$ relation there is no element with two predecessors by adding the following key dependency:

- $S(x, y) \wedge S\left(x^{\prime}, y\right) \rightarrow x=x^{\prime}$.

Note that the $S$ relation can still have a number of deviations from representing a linear order, in particular an element can have more than one distinct successor. This concludes our construction. Correctness is shown in the Appendix.

The Turing machine construction of Theorem 5.1 can be used to give an alternative proof of the undecidability of the equivalence of plain SO-tgds in the presence of source key dependencies, which was originally shown in Reference [12] by a reduction from the domino problem.

Also, we note that the SO tgd simulating a Turing machine computation can produce a core with f-blocks of arbitrary size but with bounded f-degree if the Turing machine does not halt. In this case, by Theorem 4.18, the SO tgd cannot be equivalent to a nested GLAV mapping. This immediately gives us the following undecidability result:

THEOREM 5.2. It is undecidable whether a given plain SO tgd is equivalent to a nested GLAV mapping in the presence of a single source key dependency. 
Nested GLAV mappings. We now show that in contrast, the problem of deciding whether a nested GLAV mapping is equivalent to a GLAV mapping is decidable even in the presence of source egds.

The proof strategy of Theorem 4.3 is still valid, namely, showing that the class of schema mappings has (1) effective threshold, (2) effective bounded anchor, as well as that (3) a mapping is logically equivalent to a GLAV mapping iff f-block size is bounded. We already mentioned that (3) still holds in the presence of source egds. It thus remains to show that also (1) and (2) hold if source egds are allowed.

We now show that nested tgds have effective threshold for f-block size also in the presence of source egds. However, a straightforward extension of Theorem 4.10 is not possible, as the following example illustrates:

Example 5.3. Consider the following nested $\operatorname{tgd} \sigma$ :

$$
\begin{aligned}
& \forall z\left(Q(z) \rightarrow \exists y \forall x_{1} \forall x_{2}\right. \\
& \left.\left(P_{1}\left(z, x_{1}\right) \wedge P_{2}\left(z, x_{2}\right) \rightarrow R\left(y, x_{1}, x_{2}\right)\right)\right)
\end{aligned}
$$

and the set $\Sigma_{s}$ of source dependencies given by $P_{1}\left(z, x_{1}\right) \wedge P_{1}\left(z, x_{1}^{\prime}\right) \rightarrow x_{1}=x_{1}^{\prime}$.

Now consider the source instance $I=\left\{Q(a), P_{1}(a, b), P_{2}(a, b), P_{2}(a, c)\right\}$. The proof of Theorem 4.10 depends on "cloning" parts of the source instance. Intuitively, in our example this means constructing a source instance $I^{\prime}=I \cup I[b \mapsto d]$, where $[b \mapsto d]$ denotes replacing all occurrences of $b$ by $d$. That is, we have $I^{\prime}=I \cup\left\{Q(a), P_{1}(a, d), P_{2}(a, d), P_{2}(a, c)\right\}$. But while both $I$ and $I[b \mapsto d]$ satisfy $\Sigma_{s}$, the combined instance $I^{\prime}$ does not. Indeed, $\left\{P_{1}(a, b), P_{1}(a, d)\right\}$ violates $\Sigma_{s}$.

Still, it is possible to show effective threshold also in this case. The key tool for this result and further results in this section is an adapted notion of canonical instances that takes source dependencies into account. To this end, it is convenient to allow source egds to unify two constants. Formally, this simply means that we replace one of the constants by the other. We will say that instance $K^{\prime}$ is obtained from instance $K$ by an application of egd $\epsilon$ if a firing of $\epsilon$ unifies two domain elements $b_{i}$ and $b_{j}$, which means that $K^{\prime}$ is obtained from $K$ by replacing every occurrence of $b_{j}$ in $K$ by $b_{i}$.

Definition 5.4 (Legal canonical source/target instance). Consider a nested tgd $\sigma$ defined for the source schema $\mathrm{S}$ and a target schema $\mathrm{T}$, such that a set of integrity constraints $\Sigma_{s}$ consisting of egds is associated with S. Given a pattern $p$ of $\sigma$, we define the legal canonical source, respectively, target instances $I_{p}^{s}$ and $J_{p}^{s}$, as the instances obtained from the canonical source instance $I_{p}$ and target instance $J_{p}$ as follows: Instance $I_{p}^{s}$ is obtained from $I_{p}$ by chasing it with $\Sigma_{s}$. Each unification of constants performed by this chase is also applied to the constants and instantiations of Skolem terms occurring in $J_{p}$ resulting in the instance $J_{p}^{s}$.

The following lemma is folklore, e.g., a similar property is shown in Lemma 3.4 of Reference [8]. Intuitively, it states that egd applications carry over from an instance to its homomorphic image. For the sake of completeness, we give a proof of this lemma in the Appendix.

LEMMA 5.5. Let $K_{1}$ and $K_{2}$ be instances and let $\theta$ be a c-homomorphism from $K_{1}$ to $K_{2}$. Let $\Sigma$ be a set of egds and let $K_{1}^{\prime}$ denote an instance that is obtained from $K_{1}$ by a sequence $S_{1}$ of applications of egds from $\Sigma$. Then there exists a sequence $S_{2}$ of applications of egds from $\Sigma$ to instance $K_{2}$, transforming $K_{2}$ into instance $K_{2}^{\prime}$, such that there exists a c-homomorphism $\theta^{\prime}$ from $K_{1}^{\prime}$ to $K_{2}^{\prime}$. Moreover, whenever $S_{1}$ leads to the unification of two domain elements $s_{1}, s_{2} \in \operatorname{dom}\left(K_{1}\right)$, then also $S_{2}$ leads to the unification of $\theta\left(s_{1}\right), \theta\left(s_{2}\right) \in \operatorname{dom}\left(K_{2}\right)$. 
In Section 3, the cloning of subtrees in a pattern $p$ of some nested $\operatorname{tgd} \sigma$ played an important role. Adding an isomorphic copy of some (complete) subtree in $p$ and taking the canonical source/target instance of the extended pattern was straightforward. As mentioned above, in the presence of source egds, we have to consider legal canonical source/target instances. Hence, when adding an isomorphic copy of some subtree in $p$, we have to make sure that we again get a legal canonical source instance of the extended pattern. The following two lemmas show how this can be ensured without explicitly carrying out the chase with the source egds.

Definition 5.6. Let $\sigma$ be a nested tgd. Let $K$ be a set of facts generated by a subtree $T$ of some (RC-)pattern $p$ of $\sigma$, and $\vec{a}$ be the tuple of local constants of $T$ (as defined in Section 4.1). The chase with $\Sigma_{s}$ unifies constants in $I_{p}$, transforming $I_{p}, K$, and $\vec{a}$, respectively, into the legal canonical source instance $I_{p}^{s}$, the set of facts $K^{s}$ in the canonical target instance of $p$, and the tuple of constants $\vec{a}^{s}$, which we call pseudolocal constants of $T$ (respectively, of $K^{s}$ ).

Note that due to the source egds $\Sigma_{s}$, the elements of $\vec{a}^{s}$ need not be pairwise-distinct.

LEMma 5.7. Let $\sigma$ be a nested tgd and let $\Sigma_{s}$ be a set of source egds. Let $p$ be a $k$-pattern of $\sigma$ with $k \geq 2$ and consider two isomorphic (complete) subtrees $T_{1}$ and $T_{2}$ in $p$ such that the roots of $T_{1}$ and $T_{2}$ are siblings. Let $I_{p}$ denote the canonical source instance of $p$ and let $\vec{b}$ (respectively, $\vec{c}$ ) denote the local constants used in the variable instantiations in $T_{1}$ (respectively, in $T_{2}$ ) to construct $I_{p}$. Let $I_{p}^{s}$ denote the legal, canonical source instance of $p$ and let $\vec{b}^{\prime}$ (respectively, $\left.\vec{c}^{\prime}\right)$ denote the pseudolocal constants used in the variable instantiations in $T_{1}$ (respectively, in $T_{2}$ ) to construct $I_{p}^{s}$. We write $b_{i}^{\prime}$ (respectively, $\left.c_{i}^{\prime}\right)$ to refer to the ith component of $\vec{b}^{\prime}$ (respectively, $\vec{c}^{\prime}$ ). Then $\vec{b}^{\prime}$ and $\vec{c}^{\prime}$ fulfill the following properties:

(1) for all $i, j$, if $b_{i}^{\prime}=b_{j}^{\prime}$ in $I_{p}^{s}$, then also $c_{i}^{\prime}=c_{j}^{\prime}$ holds;

(2) for all $i, j$, if $b_{i}^{\prime}=c_{j}^{\prime}$ in $I_{p}^{s}$, then also $c_{i}^{\prime}=c_{j}^{\prime}$ holds.

Note that the properties in Lemma 5.7 are symmetric in $T_{1}$ and $T_{2}$ and, hence, also in $\vec{b}^{\prime}$ and $\vec{c}^{\prime}$. Property (1) can thus be strengthened to the equivalence that $b_{i}^{\prime}=b_{j}^{\prime}$ holds in $I_{p}^{s}$ if and only if $c_{i}^{\prime}=$ $c_{j}^{\prime}$ holds. Moreover, by applying Property (1) to Property (2), we obtain that $b_{i}^{\prime}=c_{j}^{\prime}$ in $I_{p}^{s}$ implies all of the equalities $b_{i}^{\prime}=c_{i}^{\prime}=c_{j}^{\prime}=b_{j}^{\prime}$. We apply these properties to construct the legal canonical source instance after adding a clone without explicitly carrying out the chase with the source egds.

Lemma 5.8. Let $\sigma$ be a nested tgd and let $\Sigma_{s}$ be a set of source egds. Let $p$ be a k-pattern of $\sigma$ with $k \geq 2$ and consider two isomorphic (complete) subtrees $T_{1}$ and $T_{2}$ in $p$ such that the roots of $T_{1}$ and $T_{2}$ are siblings. Let $I_{p}^{s}$ denote the legal, canonical source instance of $p$ and let $\vec{b}^{\prime}$ (respectively, $\vec{c}^{\prime}$ ) denote the pseudolocal constants used in the variable instantiations in $T_{1}$ (respectively, in $T_{2}$ ) to construct $I_{p}^{s}$. As in Lemma 5.7, we write $b_{i}^{\prime}$ (respectively, $c_{i}^{\prime}$ ) to refer to the ith component of $\vec{b}^{\prime}$ (respectively, $\vec{c}^{\prime}$ ).

Let $r$ be obtained from $p$ by adding another isomorphic subtree $T_{3}$ as sibling of $T_{1}$ and $T_{2}$. Let $\vec{d}$ denote the vector of fresh, pairwise-distinct constants used in the variable instantiations of the parts in $T_{3}$ when constructing the canonical source instance $I_{r}$. Moreover, let $I_{r}^{s}$ denote the legal canonical source instance of $r$. We claim that $I_{r}^{s}$ can be obtained from $I_{p}^{s}$ by leaving the variable instantiations at all parts outside $T_{3}$ unchanged and by constructing the vector $\vec{d}^{\prime}$ of pseudolocal constants in $T_{3}$ from $\vec{d}$ as follows:

(1) for all $i$, if $b_{i}^{\prime}=c_{i}^{\prime}$, then set $d_{i}^{\prime}=b_{i}^{\prime}$;

(2) for all $i, j$, if $b_{i}^{\prime}=b_{j}^{\prime}$, then set $d_{i}^{\prime}=d_{j}^{\prime}$.

Proof. We divide the proof in two steps, showing that the unifications required according to (1) and (2) above are necessary and sufficient. Both steps rely on the one-to-one correspondence 
between the triggerings in $T_{1}, T_{2}$, and $T_{3}$ and, likewise, between the variable instantiations $\vec{b}, \vec{c}$, and $\vec{d}$ for the canonical source instance $I_{r}$.

Necessary unifications. Let $p^{\prime}$ be equal to pattern $p$ but use $\vec{b}$ and $\vec{d}$ as local constants of $T_{1}$ and $T_{2}$ to produce the canonical source instance $I_{p^{\prime}}$. Consider the c-homomorphism $\theta$ from $I_{p}$ to $I_{p^{\prime}}$ with $\theta\left(c_{i}\right)=d_{i}$ for every $i$ and $\theta(s)=s$ otherwise. Now assume that the chase of $I_{p}$ with $\Sigma$ unifies the elements $b_{i}$ and $c_{i}$. Then, by Lemma 5.5, the chase of $I_{p^{\prime}}$ with $\Sigma$ unifies the elements $\theta\left(b_{i}\right)$ and $\theta\left(c_{i}\right)$ with $\theta\left(b_{i}\right)=b_{i}$ and $\theta\left(c_{i}\right)=d_{i}$. By $I_{p^{\prime}} \subseteq I_{r}$, we thus also have in $I_{r}^{s}$ the equality $d_{i}^{\prime}=b_{i}^{\prime}$ as requested by condition (1).

To show that the unifications according to condition (2) are also necessary, we again consider pattern $p^{\prime}$ with canonical source instance $I_{p^{\prime}}$. By the isomorphism of $I_{p}$ and $I_{p^{\prime}}$, if the chase of $I_{p}$ with $\Sigma$ unifies $b_{i}$ and $b_{j}$, then so does the chase of $I_{p^{\prime}}$ with $\Sigma$. But then, by applying Lemma 5.7 to $I_{p^{\prime}}$, we conclude that $d_{i}^{\prime}=d_{j}^{\prime}$ is enforced by the chase of $I_{p^{\prime}}$. By $I_{p^{\prime}} \subseteq I_{r}$, we thus also have in $I_{r}^{s}$ the equality $d_{i}^{\prime}=d_{j}^{\prime}$ as requested by condition (2).

Sufficient unifications. The proof is indirect. Suppose that the chase of $I_{r}$ with $\Sigma$ enforces further unifications. We distinguish several cases and derive a contradiction in each of them.

Case 1. Suppose that some $d_{i}$ is unified with some $d_{j}$ although $b_{i}^{\prime} \neq b_{j}^{\prime}$ holds in $I_{p}$. Consider the chomomorphism $\theta$ from $I_{r}$ to $I_{p}$ with $\theta\left(d_{i}\right)=b_{i}$ for every $i$ and $\theta(s)=s$ otherwise. Since we assume that the chase of $I_{r}$ with $\Sigma$ unifies $d_{i}$ with $d_{j}$, the chase $I_{p}$ with $\Sigma$ unifies $b_{i}$ with $b_{j}$ by Lemma 5.5. This contradicts our assumption that $b_{i}^{\prime} \neq b_{j}^{\prime}$ holds in $I_{p}^{s}$.

Case 2. Suppose that some $d_{i}$ is unified with $b_{i}$ (or, symmetrically with some $c_{i}$ ) although this equality is not enforced by conditions (1) and (2). In particular, this means that $b_{i}^{\prime} \neq c_{i}^{\prime}$ in $I_{p}$. Consider the c-homomorphism $\theta$ from $I_{r}$ to $I_{p}$ with $\theta\left(d_{i}\right)=c_{i}$ for every $i$ and $\theta(s)=s$ otherwise. By Lemma 5.5 and by condition $d_{i}^{\prime}=b_{i}^{\prime}$ in $I_{r}^{s}$, we conclude that $c_{i}^{\prime}=b_{i}^{\prime}$ holds in $I_{p}^{s}$-a contradiction.

Case 3. Suppose that some $d_{i}$ is unified with some $b_{j}$ (or, symmetrically with some $c_{j}$ ) with $i \neq j$ although this equality is not enforced by conditions (1) and (2). Again, we consider the chomomorphism $\theta$ from $I_{r}$ to $I_{p}$ with $\theta\left(d_{i}\right)=c_{i}$ for every $i$ and $\theta(s)=s$ otherwise. By Lemma 5.5 and by condition $d_{i}^{\prime}=b_{j}^{\prime}$ in $I_{r}^{s}$, we conclude that $c_{i}^{\prime}=b_{j}^{\prime}$ holds in $I_{p}^{s}$. By Lemma 5.7, all the equalities $c_{i}^{\prime}=b_{i}^{\prime}=b_{j}^{\prime}=c_{j}^{\prime}$ hold in $I_{p}^{s}$. But then condition (1) is applicable and enforces the equalities $d_{i}^{\prime}=b_{i}^{\prime}$ and $d_{j}^{\prime}=b_{j}^{\prime}$. Together with $b_{i}^{\prime}=b_{j}^{\prime}$, also the equality $d_{i}^{\prime}=b_{j}^{\prime}$ is thus enforced by condition (1)-a contradiction.

Case 4. Suppose that some $d_{i}$ is unified with some $t$ in $\operatorname{dom}\left(I_{r}\right)$ outside $\vec{b}, \vec{c}$, and $\vec{d}$, although this equality is not enforced by conditions (1) and (2). We consider two c-homomorphisms $\theta_{1}$ and $\theta_{2}$ from $I_{r}$ to $I_{p}$ with $\theta_{1}\left(d_{i}\right)=b_{i}$ and $\theta_{2}\left(d_{i}\right)=c_{i}$ for every $i$ and $\theta_{1}(s)=s$ and $\theta_{2}(s)=s$ otherwise. We are assuming that $d_{i}$ is unified with some $t$ outside $\vec{b}, \vec{c}$, and $\vec{d}$ by the chase of $I_{r}$ with $\Sigma$. By applying Lemma 5.5 to $\theta_{1}$, we conclude that $c_{i}$ is unified with $t$ by the chase of $I_{p}$ with $\Sigma$. By applying Lemma 5.5 to $\theta_{2}$, we conclude that also $b_{i}$ is unified with $t$ by the chase of $I_{p}$ with $\Sigma$. But then, in total, $b_{i}$ is unified with $c_{i}$ by the chase of $I_{p}$ with $\Sigma$. Hence, by condition (1), we set $d_{i}^{\prime}=b_{i}^{\prime}$. Together with $b_{i}^{\prime}=s$, we conclude that the unification of $d_{i}$ with $s$ is enforced by conditions (1) and (2).

Before we can lift Theorem 4.10 to the setting with source egds, we observe that the above definitions and properties of legal canonical source instances can be literally carried over from patterns to RC-patterns introduced in Definition 4.7. We are now able to show our desired result.

THEOREM 5.9. The class of nested tgds with source egds has effective threshold for f-block size. 
Proof. The proof proceeds analogously to the proof of Theorem 4.10-replacing canonical instances by legal canonical instances. Below, we describe how the proof of Theorem 4.10 has to be adapted to take the set $\Sigma_{s}$ of source egds into account.

Again, by Lemma 4.5, it suffices to consider a single nested $\operatorname{tgd} \sigma$. Moreover, let $\sigma$ be an arbitrary nested $\operatorname{tgd} \sigma$ and let $n$ denote the maximum size of all RC-1-patterns of $\sigma$. Then, we again set the threshold $g(\sigma)$ to $g(\sigma)=n+1$. Now suppose that there exists a source instance $I$ with $I=\Sigma$, s.t. $\operatorname{core}(\operatorname{chase}(I, \sigma))$ has f-block size $m \geq g(\sigma)$. As in the proof of Theorem 4.10, it suffices to show that, from $I$, we can construct a source instance $I^{\prime}$ with $I^{\prime} \mid=\Sigma$, s.t. $\operatorname{core}\left(\operatorname{chase}\left(I^{\prime}, \sigma\right)\right)$ has f-block size $\geq m+1$.

Considering legal canonical source instances only. By Corollary 3.15, we know that the facts in some f-block $B$ of $\operatorname{core}(\operatorname{chase}(I, \sigma))$ must be generated by a single chase tree in the chase forest $\mathcal{F}_{\sigma, I}$. Now suppose that f-block size $m$ of $\operatorname{core}(\operatorname{chase}(I, \sigma))$ is realized by some f-block $B$ of $\operatorname{core}(\operatorname{chase}(I, \sigma))$ and that the facts in $B$ are generated by RC-chase tree $T_{p}$ with RC-pattern $p$. Let $I_{p}^{s}$ be the legal canonical source instance of $p$. Then the following property holds:

Claim 1. The f-block size of $\operatorname{core}\left(\operatorname{chase}\left(I_{p}^{s}, \sigma\right)\right)$ is at least $m$.

The proof idea of this claim is the same as in the proof of Theorem 4.10. We just need now in add Lemma 5.5 to conclude that there exists a c-homomorphism from $I_{p}^{s}$ to $I$ and that there again is a one-to-one correspondence between the triggerings in (and the atoms generated by) the chase tree $T_{\sigma, I}$ and the corresponding pattern $p$ equipped with the variable bindings according to the legal canonical source instance $I_{p}^{s}$. We can thus again define the set $B_{p}$ of atoms in the legal canonical target instance corresponding to the set $B$ in chase $(I, \sigma)$. As in the proof of Theorem 4.10, it can be shown that $B_{p}$ or a superset thereof must also be an f-block in the core of the canonical target instance. In the remainder of the proof, we may thus assume w.l.o.g. that $I$ is the legal canonical source instance of $p$.

Isomorphic subtrees in $T_{p}$ with local constants in atoms of $B$. As in the proof of Theorem 4.10, the considerations on the threshold $g(\sigma)$ allow us to conclude that there exist (at least) two siblings in the pattern $p$, which are the roots of isomorphic subtrees $T_{1}$ and $T_{2}$, such that both $T_{1}$ and $T_{2}$ generate some facts of $B$. Note that now that we are considering legal canonical source instances, it may happen that local constants of subtrees $T_{1}$ and $T_{2}$ are possibly unified by the source chase. We therefore strengthen Claim 2 from the proof of Theorem 4.10 to rule out such unifications.

Claim 2. For each $i \in\{1,2\}$, there exists an atom $A_{i}$ generated by $T_{i}$ such that $A_{i} \in B$, atom $A_{i}$ contains a pseudolocal constant $b_{i}$ of $T_{i}$, and $b_{1}$ and $b_{2}$ are not unified by the chase with the source egds.

Again, the proof idea is the same as in the proof of Theorem 4.10, i.e., if no such atoms $A_{1}$ and $A_{2}$ existed, then we would derive a contradiction by exhibiting an endomorphism on chase $(\sigma, I)$, which further shrinks the core.

Construct another isomorphic subtree. Let $\vec{b}$ denote the pseudolocal constants of $T_{1}$ and let $\vec{c}$ denote the pseudolocal constants of $T_{2}$. By Lemma 5.8, we know which constant unifications have to be carried out when adding another clone $T_{3}$ with pseudolocal constants $\vec{d}$. Let $\theta$ be the mapping that realizes the one-to-one correspondence between $\vec{b}$ and $\vec{d}$, i.e., $\theta\left(b_{i}\right)=d_{i}$ for every $b_{i}$ in $\vec{b}$ and $d_{i}$ in $\vec{d}$. We extend $I$ to $I^{\prime}=I \cup \theta(I)$. For the f-block size of $\operatorname{core}\left(\operatorname{chase}\left(I^{\prime}, \sigma\right)\right)$, the following property can be shown:

Claim 3. The instance core $\left(\operatorname{chase}\left(I^{\prime}, \sigma\right)\right)$ has f-block size $\geq m+1$.

Again, the proof of this claim follows the same pattern as the proof of Claim 3 in the proof of Theorem 4.10, 
Lemma 5.8 shows how legal canonical instances of a pattern $p$ can be obtained via extensions of respective legal canonical instances of a smaller pattern $p^{\prime} \leq_{2} p$, exactly as in the case when no source egds are present. With this provision, we can lift Lemma 3.22 to the case of source egds.

\section{LEMMA 5.10. Consider the following setting:}

- A set $\sum$ of nested tgds and a nested $\operatorname{tg} d \sigma$.

- Integerk $>m_{\sigma} w_{\Sigma}$, where $m_{\sigma}$ is the maximum number of nulls in the canonical target instances of 1-patterns of $\sigma$ and $w_{\Sigma}$ is the maximum number of universally quantified variables in any nested tgd in $\Sigma$.

- Patterns $p^{k}, p$ of $\sigma$ where $p^{k}$ is a $k$-pattern and $p^{k} \leq_{k} p$ holds.

- Pairs $\left(I_{p^{k}}^{s}, J_{p^{k}}^{s}\right)$ and $\left(I_{p}^{s}, J_{p}^{s}\right)$ of the legal canonical source and the legal canonical target instances of the patterns $p^{k}$ and $p$, respectively. We assume that $I_{p}^{s}$ and $J_{p}^{s}$ have been constructed by extending $I_{p^{k}}^{s}$ and $J_{p^{k}}^{s}$, as specified in Lemma 5.8.

Then, every homomorphism $h^{k}$ from $J_{p^{k}}$ to chase $\left(I_{p^{k}}, \Sigma\right)$ can be extended to a homomorphism $h$ from $J_{p}$ to chase $\left(I_{p}, \Sigma\right)$.

Proof. As in the case of the original Lemma 3.22, the proof is by induction on the length $i$ of the sequence of $k^{+}$-increments of $p^{k}$ transforming it to $p$, with the trivial base case $i=0$. Assume that for some sequence length $i$, a homomorphism $h_{i}$ from $J_{p_{i}}^{s}$ to $\operatorname{chase}\left(I_{p_{i}}^{s}, \Sigma\right)$ exists, s.t. $h_{i}$ extends $h^{k}$. We show that $h_{i}$ can be transformed to a homomorphism $h_{i+1}$ from $J_{p_{i+1}}^{s}$ to $\operatorname{chase}\left(I_{p_{i+1}}^{s}, \Sigma\right)$, where $p_{i+1}$ is obtained from $p_{i}$ by a single $k^{+}$-increment.

We thus have trees $T_{1}, \ldots, T_{k}$ in $p_{i}$ that are instantiated, in the legal canonical target instance $J_{p_{i}}^{s}$, by the subinstances $K_{1}, \ldots, K_{k}$. In particular, if $T_{1}, \ldots, T_{k}$ are rooted at nodes with the label $\sigma_{i}$, the trees $T_{1}, \ldots, T_{k}$ represent chase trees resulting from the triggerings of the part $\sigma_{i}$ with the input assignment $\vec{a}_{0}$. By $\vec{a}_{1}, \ldots, \vec{a}_{k}$, we denote tuples of pseudolocal constants of $T_{1}, \ldots, T_{k}$.

The pattern $p_{i+1}$ adds another clone $T_{k+1}$ to the subtrees $T_{1}, \ldots, T_{k}$. In the legal canonical target instance, this corresponds to an addition of a further subinstance $K_{k+1}$ with a tuple of pseudolocal constants $\vec{a}_{k+1}$.

Let $F$ denote the set of nulls shared between any two distinct instances in the set $\left\{K_{1}, \ldots, K_{k+1}\right\}$. In the absence of egds, in the proof of Lemma 3.22, we argued that each such shared null has the form $\hat{f}(\vec{b})$ where $\vec{b}$ is a prefix of the common input assignment $\vec{a}_{0}$ of the triggerings corresponding to the roots of $T_{1}, \ldots, T_{k+1}$. The effect of the source egds, however, may be a unification of constants $a_{r}$ in $\vec{a}_{j}$ with $a_{\ell}^{\prime}$ in $\vec{a}_{j^{\prime}}$. Source egds may thus also lead to the unification of a null $\hat{f}\left(\vec{a}_{0} \cdot \vec{a}_{j}\right)$ in $K_{j}$ with a null $\hat{f}\left(\vec{a}_{0} \cdot \vec{a}_{j^{\prime}}\right)$ in $K_{j^{\prime}}$. Hence, in the presence of source egds, the set $F$ may be significantly bigger than the number of distinct Skolem functions $v_{\sigma}$. However, Lemma 5.8 allows us to derive an upper bound on $|F|$ also in this case. Indeed, Lemma 5.8 establishes precisely the form of the tuple of pseudolocal constants $\vec{a}_{k+1}$ of $K_{k+1}$. More specifically, if constants are positions $r$ and $\ell$ are unified in $\vec{a}_{j}$ and $\vec{a}_{j^{\prime \prime}}$, then the positions $r$ and $\ell$ contain the same constant in all tuples $\vec{a}_{1}, \ldots, \vec{a}_{k+1}$. By the same token, we may conclude that the unification of nulls $\hat{f}\left(\vec{a}_{0} \cdot \vec{a}_{k+1}\right)$ in $K_{k+1}$ and $\hat{f}\left(\vec{a}_{0} \cdot \vec{a}_{j^{\prime}}\right)$ in $K_{j^{\prime}}$ is only possible if these nulls are also unified with the corresponding null $\hat{f}\left(\vec{a}_{0} \cdot \vec{a}_{j}\right)$ in $K_{j}$. In other words, the number of nulls created by ancestor triggerings of $T_{1}, \ldots, T_{n}$ plus the number of nulls in $K_{j}$ give us an upper bound on the number of nulls shared between any two distinct subinstances $K_{j}$ and $K_{j^{\prime}}$. That is, additional nulls contained in clones can be neglected for this upper bound. But then we have $|F| \leq m_{\sigma}$. By Proposition 3.8, this maximum $m_{\sigma}$ exists and can be computed. 
Having obtained a new upper bound on $|F|$, the remainder of the proof of Lemma 3.22 can be easily carried over to the setting with source egds.

Applying Lemma 5.10 instead of Lemma 3.22, the argumentation in the proof of Theorem 4.14 that nested tgds have effective bounded anchor still holds in the presence of source egds by using legal canonical instances. We can thus extend Theorem 4.3:

THEOREM 5.11. It is decidable whether a given nested GLAV mapping is equivalent to a GLAV mapping in the presence of source egds.

Finally, we show that also the implication is decidable in the presence of source egds.

THEOREM 5.12. The implication problem for nested tgds is decidable in the presence of source egds, i.e., the following problem is decidable: Given source and target schemas $\mathbf{S}, \mathbf{T}$, a set $\Sigma_{s}$ of source egds, a set $\Sigma$ of nested tgds, and a nested $\operatorname{tg} d \sigma$, does $\Sigma_{s} \cup \Sigma \mid=\sigma$ hold?

Proof. The proof uses exactly the same ideas as the proof of Theorem 3.1 (i.e, decidability of implication without source constraints) with two crucial modifications:

(1) we have to use legal canonical (source and target) instances rather than arbitrary canonical instances;

(2) the upper bound $k$ on the $k$-patterns to be inspected in the procedure IMPLIES in Section 3 has to be increased to match the precondition of Lemma 5.10.

The proof is analogous to the proof of Theorem 3.1:

Characterization of implication via the existence of homomorphisms. The implication $\Sigma_{s} \cup \Sigma \mid=\sigma$ holds if every instance $I$ over source schema $\mathbf{S}$ satisfying $\Sigma_{s}$ and $\Sigma$ also satisfies $\sigma$. Analogously to the case without source dependencies, the latter condition is equivalent to the following: For every instance $I$ over source schema $\mathbf{S}$ satisfying $\Sigma_{s}$, there exists a homomorphism from chase $(I, \sigma)$ to chase $(I, \Sigma)$. In other words, the source egds only play a role in restricting the set of source instances $I$ to be considered by this implication criterion. The criterion itself (namely, characterizing implication via the existence of homomorphisms between the chase results of $\sigma$ and the chase results of $\Sigma$ ) is not affected.

Considering legal canonical source instances only. Recall that, in Lemma 3.18, we have shown that it suffices to inspect canonical source instances $I$ rather than all source instances over a given source schema. Analogously, we can show that the following equivalence holds: Every instance $I$ over source schema $\mathbf{S}$ satisfying $\Sigma_{s}$ and $\Sigma$ also satisfies $\sigma$ if and only if every legal canonical source instance over source schema $\mathbf{S}$ satisfying $\Sigma$ also satisfies $\sigma$. Clearly, we can drop the condition that $\Sigma_{s}$ must be satisfied, because every legal canonical source instance is guaranteed to do so. The extension of the proof of Lemma 3.18 to the setting with source egds is straightforward.

Considering only $\boldsymbol{k}$-patterns up to some fixed $\boldsymbol{k}$. This is by virtue of Lemma 5.10.

Definition of a decision procedure. To sum up, we can define a decision procedure IMPLIES ${ }^{s}$ for the implication problem of nested tgds in the presence of source egds by modifying the algorithm ImPLies from Section 3 in two ways: (1) rather than defining $k=v_{\sigma} \cdot w_{\Sigma}+1$, we set $k=m_{\sigma} \cdot w_{\Sigma}+1$, where $m_{\sigma}$ denotes the maximum number of nulls in the target canonical instance of any 1-pattern of $\sigma$; and (2) we use legal canonical instances of $k$-patterns in place of canonical instances.

Theorem 5.12 separates the complexity of reasoning for nested and plain SO tgds, since as shown in Reference [12], equivalence-and thus also implication-of plain SO tgds is undecidable even in the presence of a single source key dependency. 


\section{CONCLUDING REMARKS}

In this article, we initiated the study of fundamental reasoning tasks and structural properties of nested tgds. On the positive side, we showed that the following problems are decidable: the implication problem (and, hence, the equivalence problem) of nested tgds, and the problem of deciding whether a given nested GLAV mapping is equivalent to some GLAV mapping. We also showed that these problems remain decidable even if source egds are allowed. In contrast, we established that the problem whether a given plain SO tgd is equivalent to some GLAV mapping becomes undecidable as soon as a single key dependency is allowed in the source schema.

For future work, the decidability of the equivalence problem for plain SO tgds and of the problem of determining whether a given plain SO tgd is equivalent to some GLAV mapping (respectively, to some nested GLAV mapping) remains open. Moreover, the aforementioned decidability results for nested tgds call for further study: All of our decidability results depend on the notion of $k$ patterns introduced in Section 3. As pointed out in that section, the number of $k$-patterns and the maximum size of $k$-patterns for a given nested tgd are non-elementary in the depth of the nested tgd, and so are all algorithms utilizing patterns. It is an important open question for future work to find out whether this high complexity is inherent in these problems or more efficient algorithms can be designed. Lemma 3.22 seems to be the key to investigating this question: There, we managed to prove that any homomorphism can be extended from a $k$-pattern $p^{k}$ to a pattern $p$ obtained by an arbitrary sequence of $k^{+}$-increments provided that $k$ is chosen sufficiently big. It remains to be analyzed if such an extension of homomorphisms can also be guaranteed for smaller patterns.

Another direction for future research is concerned with structural characterizations of schema mappings along the lines of Reference [28]. In this article, we discovered necessary conditions (via the notions of unbounded f-degree and bounded path length) of schema mappings that are logically equivalent to some nested GLAV mapping. These properties sometimes provide an easy argument for telling apart plain SO tgds from nested GLAV mappings. It remains open whether these properties can be extended to a sufficient condition. For instance, are all plain SO tgds with unbounded f-degree and/or bounded path length equivalent to a nested GLAV mapping? A structural characterization of plain SO tgds (raised in Reference [2]) also remains an interesting open problem.

\section{APPENDICES}

\section{A PROOFS FOR SECTION 4}

We first complete the proof of Theorem 4.10:

THEOREM 4.10 The class of nested GLAV mappings has effective threshold for f-block size.

Proof. In the main body of the text, we have already presented the overall proof, which was based on the following three claims:

CLAim 1. The f-block size of core $\left(\right.$ chase $\left.\left(I_{p}, \sigma\right)\right)$ is at least $m$.

Claim 2. For each $i \in\{1,2\}$, there exists an atom $A_{i}$ generated by $T_{i}$ such that $A_{i} \in B$ and $A_{i}$ contains a local constant of $T_{i}$. (Recall that in a canonical target instance, local constants do not occur outside a subinstance generated by a subtree of a chase tree.)

CLAIm 3. The instance core $\left(\right.$ chase $\left.\left(I^{\prime}, \sigma\right)\right)$ has f-block size $\geq m+1$.

It only remains to prove these claims. 
Proof of Claim 1. Let $B$ be an f-block of size $m$ of core $(\operatorname{chase}(I, \sigma))$ and suppose that the facts in $B$ are generated by RC-chase tree $T_{p}$ with RC-pattern $p$. Moreover, let $I_{p}$ be the canonical source instance of $p$. Recall from the proof of Lemma 3.18 that there is a one-to-one correspondence $\theta^{[\mathrm{tr}]}$ between the triggerings in $T_{p}$ and those in the canonical chase tree (i.e., the chase tree obtained from $p$ assuming that variables are instantiated to fresh, pairwise-distinct constants). Clearly, this correspondence also carries over to chase trees with restricted conclusions. Moreover, there is a c-homomorphism $\theta$ from $I_{p}$ to $I$, which is also a c-homomorphism from $J_{p}$ (the canonical target instance of the RC-pattern $p)$ to $B$. Specifically, for every triggering $\left(\sigma_{i}, \vec{a}_{0}, \vec{a}\right)$ in $T_{p}$ and triggering $\left(\sigma_{i}, \vec{a}_{0}^{\prime}, \vec{a}^{\prime}\right)$ in the canonical chase tree such that $\theta^{[\operatorname{tr}]}\left(\left(\sigma_{i}, \vec{a}_{0}^{\prime}, \vec{a}^{\prime}\right)\right)=\left(\sigma_{i}, \vec{a}_{0}, \vec{a}\right)$, mapping $\theta$ maps the elements of $\vec{a}^{\prime}$ and $\vec{a}_{0}^{\prime}$ to the respective elements of $\vec{a}$ and $\vec{a}_{0}$.

Recall also the mapping $\theta^{\text {[null] }}$ on nulls of $J_{p}$, which sends each null $\hat{f}\left(\vec{a}_{0}^{\prime} \cdot \vec{a}^{\prime}\right) \in \operatorname{nulls}\left(\left(\sigma_{i}, \vec{a}_{0}^{\prime}, \vec{a}^{\prime}\right)\right)$ to a distinct null $\hat{f}\left(\vec{a}_{0} \cdot \vec{a}\right) \in \operatorname{nulls}\left(\left(\sigma_{i}, \vec{a}_{0}, \vec{a}\right)\right)$. In other words, $\theta^{\text {[null] }}: N_{p} \rightarrow N$ is a bijection between $N_{p}$ and $N$, where we write $N_{p}$ and $N$ to denote the sets of nulls in $J_{p}$ and in $B$, respectively. Mapping $\theta^{[\text {null] }}$ is bijective even in the case when $\theta$ is not injective. As in the proof of Lemma 3.18, we write $\theta^{\overline{[\text { null }]}}$ to denote the inverse of $\theta^{\text {[null }]}$.

We will now show that $J_{p}$ is connected in (the Gaifman graph of facts of) $\operatorname{chase}\left(I_{p}, \sigma\right)$ and that $J_{p}$ persists (up to variable renaming) in core $\left(\operatorname{chase}\left(I_{p}, \sigma\right)\right)$. By $J_{p}=\theta^{-1}(B)$, we have $\left|J_{p}\right| \geq m$. In total, we may thus conclude that $\operatorname{core}\left(\operatorname{chase}\left(I_{p}, \sigma\right)\right)$ has f-block size at least $m$. To see that $J_{p}$ is indeed connected, we observe that the c-homomorphism $\theta$ from $J_{p}$ to $B$ coincides with $\theta^{\text {[null] }}$ when restricted to the nulls in $J_{p}$ and $B$, respectively. By the bijectivity of $\theta^{\text {[null] }}, \theta^{\text {[null] }}$ (and, hence, also $\left.\theta^{\overline{[\text { null }}}\right)$ is a variable renaming. But then the connectedness of $B$ implies the connectedness of $J_{p}=\theta^{-1}(B)$.

It remains to show that $J_{p}$ persists (up to variable renaming) in core (chase $\left.\left(I_{p}, \sigma\right)\right)$. To this end, it suffices to show that every homomorphism $h$ from $J_{p}$ to $\operatorname{chase}\left(I_{p}, \sigma\right)$ is a variable renaming. Suppose to the contrary that there exists a homomorphism $h$ from $J_{p}$ to $\operatorname{chase}\left(I_{p}, \sigma\right)$ that either maps some null to a constant or maps two distinct nulls to the same null. From $h$, we construct the homomorphism $e$ from $B$ to chase $(I, \sigma)$ as in the proof of Lemma 3.18, namely:

$$
e(s)= \begin{cases}s, & \text { if } s \text { is a constant } \\ \theta^{[\text {null }]}\left(h\left(\theta^{\overline{[\text { null }]}}(s)\right)\right), & \text { if } s \text { is a null. }\end{cases}
$$

As in the proof of Lemma 3.18, it can be shown that $e$ is indeed a homomorphism from $B$ to chase $(I, \sigma)$. But then, since we are assuming that $h$ is not a variable renaming, we conclude that $e$ is not a variable renaming either. This means that $B$ can be further reduced in core $(\operatorname{chase}(I, \sigma))$, which contradicts our original assumption that $B$ is an f-block in core $(\operatorname{chase}(I, \sigma))$.

Proof of ClAim 2. In the remainder of the proof, we assume w.l.o.g. that $I$ is the canonical source instance of $p$. Let $B$ be an f-block in core $(\operatorname{chase}(I, \sigma))$ of size $m$ and let $T_{1}$ and $T_{2}$ be two isomorphic subtrees in the RC-pattern $p$, such that the roots of $T_{1}$ and $T_{2}$ are siblings. By the isomorphism between $T_{1}$ and $T_{2}$, there exists a one-to-one correspondence between the facts generated by $T_{1}$ and the facts generated by $T_{2}$. Moreover, there exists a c-homomorphism $\rho$ between the set of facts generated by $T_{1}$ and the set of facts generated by $T_{2}$. Since we are dealing with the canonical source instance, $\rho$ is in fact bijective.

We have to show that, for each $i \in\{1,2\}$, there exists an atom $A_{i}$ generated by $T_{i}$ such that $A_{i} \in B$ and $A_{i}$ contains a local constant of $T_{i}$. Suppose to the contrary that in the facts generated by one of the $T_{i}$ 's, the local constants only occur (if at all) as arguments of Skolem terms in these facts. By the existence of the c-homomorphism $\rho$, which is in fact a c-isomorphism, it follows that 
then none of the facts generated by either $T_{1}$ or $T_{2}$ contains a local constant as argument. But then $\rho$ is in fact a homomorphism (leaving constants unchanged) and not just a c-homomorphism.

We now define the endomorphism $\rho^{\prime}$ on $B$ such that $\rho^{\prime}(s)=\rho(s)$ if $s$ is a null $\hat{f}(\vec{a})$ such that a local constant generated by $T_{1}$ occurs in $\vec{a}$, and $\rho^{\prime}(s)=s$ otherwise. Clearly, $\rho^{\prime}$ is a homomorphism (and, hence, an endomorphism), because it maps every atom generated by $T_{1}$ to an atom generated by $T_{2}$ and maps all other atoms to themselves. But the existence of such a proper endomorphism $\rho^{\prime}$ contradicts the assumption that $B$ is an f-block in core $(\operatorname{chase}(I, \sigma))$.

Proof of Claim 3. We partition the atoms in $B$ into three subsets $\mathcal{A}, \mathcal{B}, C$, such that $\mathcal{B}$ contains the atoms generated by $T_{1}, C$ contains the atoms generated by $T_{2}$, and $\mathcal{A}$ contains the remaining atoms of $B$. Let $\vec{b}$ denote the local constants of $T_{1}$ and let $\vec{c}$ denote the local constants of $T_{2}$. Then $\rho$ from the proof of Claim 2 maps each constant $b_{i}$ in $\vec{b}$ to the corresponding constant $c_{i}$ in $\vec{c}$. Let $\vec{a}$ denote the local constants of the atoms generated by any ancestor triggering of the roots of $T_{1}$ and of $T_{2}$. Then the atoms generated by $T_{1}$ (respectively, $T_{2}$ ) are of the form $R[\vec{a}, \vec{b}]$ (respectively, $R[\vec{a}, \vec{c}]$ ), i.e.: the arguments in these facts are either constants in $\vec{a}$ and $\vec{b}$ (respectively, in $\vec{a}$ and $\vec{c}$ ) or nulls resulting from instantiating the arguments of Skolem terms by constants in $\vec{a}$ and $\vec{b}$ (respectively, in $\vec{a}$ and $\vec{c}$ ). In particular, by Claim 2, there indeed exists an atom $A_{1}$ (respectively, $A_{2}$ ) of the form $R[\vec{a}, \vec{b}]$ (respectively, $R[\vec{a}, \vec{c}]$ ), such that at least one constant $b_{i}$ in $\vec{b}$ (respectively, $c_{i}$ in $\vec{c}$ ) occurs as argument in this atom.

We now introduce a new vector $\vec{d}$ of fresh, pairwise-distinct constants, such that $\vec{d}$ has the same size as $\vec{b}$ and $\vec{c}$. Moreover, we define a mapping $\theta$ that maps every $b_{i}$ in $\vec{b}$ to the corresponding constant $d_{i}$ in $\vec{d}$. Let $\theta(I)$ be the instance obtained from $I$ by applying $\theta$ to the constants occurring in the facts of $I$. Moreover, we set $I^{\prime}=I \cup \theta(I)$. We claim that $I^{\prime}$ has the desired property according to Claim 3, namely: $\operatorname{core}\left(\operatorname{chase}\left(I^{\prime}, \sigma\right)\right)$ has f-block size $\geq m+1$.

Before we prove this claim, we make some observations and introduce some more terminology. Let $J=\operatorname{chase}(I, \sigma)$ and $J^{\prime}=\operatorname{chase}\left(I^{\prime}, \sigma\right)$. We observe that $\theta$ can be extended to a c-homomorphism from $J$ to $J^{\prime}$ by applying $\theta$ also to the constants used to instantiate the arguments of Skolem terms. By slight abuse of notation, we also use $\theta$ to refer to this c-homomorphism. Indeed, let $F$ be a fact of $J$. We have to show that $\theta(F) \in J^{\prime}$. Let $\sigma_{i}$ be the nested tgd part whose firing in the chase of $I$ generates $F$ and let $I_{F}$ be the facts from $I$ that trigger the firing of $\sigma_{i}$ and all its ancestor parts. Let $I_{F}^{\prime}=\theta\left(I_{F}\right)$. It is clear that $I_{F}^{\prime} \subseteq I^{\prime}$ holds by construction of $I^{\prime}$. But then $\sigma_{i}$ also fires on $I_{F}^{\prime}$ generating $\theta(F)$. Thus, $\theta(F) \in J^{\prime}$, which was to be shown.

Moreover, we can define a c-homomorphism $\bar{\theta}$ also in the opposite direction, i.e., from $J^{\prime}$ to $J$. To this end, we define $\bar{\theta}$ as follows:

$$
\bar{\theta}(s)= \begin{cases}s, & \text { if } s \in \operatorname{const}(J) \\ \theta^{-1}(s), & \text { if } s \in \operatorname{const}\left(J^{\prime}\right) \backslash \operatorname{const}(J) \\ \hat{f}\left(\theta^{-1}(\vec{e})\right), & \text { if } s \text { is a null of the form } \hat{f}(\vec{e}) .\end{cases}
$$

In the above definition, we write $\theta^{-1}(\vec{e})$ to denote the application of $\theta^{-1}$ to every component of vector $\vec{e}$. To show that $\bar{\theta}$ is a c-homomorphism, let $F^{\prime}$ be a fact in $J^{\prime}$. We have to show that $\bar{\theta}\left(F^{\prime}\right) \in J$. Let $\sigma_{i}$ be the nested tgd part whose firing generates $F^{\prime}$ in the chase of $I^{\prime}$ with $\sigma$. Let $I_{F^{\prime}}^{\prime}$ be the facts from $I^{\prime}$ that trigger the firing of $\sigma_{i}$ and of all its ancestor parts. Let $I_{F}=\theta^{-1}\left(I_{F^{\prime}}^{\prime}\right)$. It is clear that $I_{F} \subseteq I$ by construction of $I^{\prime}$ as $I^{\prime}=I \cup I \theta$. But then $\sigma_{i}$ also triggers on $I_{F}$ in the chase of $I$ with $\sigma$, and this triggering generates $\bar{\theta}\left(F^{\prime}\right)$. Thus, $\bar{\theta}\left(F^{\prime}\right) \in J$, which was to be shown.

Now let us define $\mathcal{D}:=\theta(\mathcal{B})$. By the above observations, we clearly have $\mathcal{A} \cup \mathcal{B} \cup C \cup \mathcal{D} \subseteq J^{\prime}$. We now prove Claim 3 by showing several properties of $\mathcal{A} \cup \mathcal{B} \cup C \cup \mathcal{D}$.

Property 1. $\mathcal{A} \cup \mathcal{B} \cup C \cup \mathcal{D}$ is connected in $J^{\prime}$. 
Proof of Property 1. By assumption, $\mathcal{A} \cup \mathcal{B} \cup \mathcal{C}$ is an f-block and, hence, connected. It follows from Lemma 3.14 that the connection of $\mathcal{B}$ and $C$ is realized via nulls of the form $\hat{f}(\vec{a})$ occurring both in the atoms of $\mathcal{B}$ and of $C$, where $\vec{a}$ is a vector of constants used in the variable instantiations of the triggerings at the joint ancestors of the roots of $T_{1}$ and $T_{2}$. But then all these nulls of the form $\hat{f}(\vec{a})$ are also generated in $\mathcal{D}$ in the chase of $I^{\prime}$. Hence, the set $\mathcal{D}$ of atoms is connected to $\mathcal{B}$ and $C$.

Property 2. Every endomorphism $h^{\prime}$ on $J^{\prime}$ is a variable renaming on $\mathcal{A} \cup \mathcal{B} \cup C$, i.e., $h^{\prime}$ maps no null in $\mathcal{A} \cup \mathcal{B} \cup \mathcal{C}$ to a constant and $h^{\prime}$ maps no two nulls in $\mathcal{A} \cup \mathcal{B} \cup C$ to the same null.

Proof of Property 2. For the proof of both Properties 2 and 3, we make use of the following observation: Consider an arbitrary endomorphism $h$ on $J$. Then $h$ is a variable renaming on the f-block $B$. Indeed, let $g$ be the endomorphism on $J$ that reduces $J$ to the core, i.e., $C=\operatorname{core}(\operatorname{chase}(I, \sigma))=g(J)$. Now suppose that $h$ is not a variable renaming on $B$, i.e., it either maps some null in $B$ to a constant or it maps two nulls in $B$ to the same null. We can consider the composition $g(h(\cdot))$ as a homomorphism from $B$ to $J$. Clearly, $g(h(\cdot))$ is not a variable renaming on $B$ either. By definition of f-blocks, the nulls in $B$ form a connected component. Hence, we can define an endomorphism $e$ on $C$ by setting $e(s)=g(h(s))$ for all nulls $s$ in $B$ and $e(s)=s$ otherwise. Since $h$ either maps some null in $B$ to a constant or it maps two nulls in $B$ to the same null, so does $e$. But then $e$ further reduces $B$, which contradicts the assumption that $B$ is an f-block in $\operatorname{core}(\operatorname{chase}(I, \sigma))$.

We now prove Property 2 indirectly: Suppose that there exists an endomorphism $h^{\prime}$ on $J^{\prime}$ that is not a variable renaming on $\mathcal{A} \cup \mathcal{B} \cup C$. By restricting $h^{\prime}$, we can consider $h^{\prime}$ as a homomorphism from $\mathcal{A} \cup \mathcal{B} \cup C$ to $J^{\prime}$. Moreover, we can compose $h^{\prime}$ with $\bar{\theta}$ from above to get a c-homomorphism $\bar{\theta}\left(h^{\prime}(\cdot)\right)$ from $\mathcal{A} \cup \mathcal{B} \cup \mathcal{C}$ to $J$. To complete the proof, it suffices to show that $\bar{\theta}\left(h^{\prime}(\cdot)\right)$ is in fact a homomorphism, i.e., it is the identity on constants. It is easy to verify that this is the case: Let $s$ be a constant occurring in $\mathcal{A} \cup \mathcal{B} \cup C$. By $\mathcal{A} \cup \mathcal{B} \cup C \subseteq J$, we have $s \in \operatorname{const}(J)$. By the definition of homomorphisms, we have $h^{\prime}(s)=s$. Hence, by the definition of $\bar{\theta}$, we also have $\bar{\theta}\left(h^{\prime}(s)\right)=s$.

Since $h^{\prime}$ is not a variable renaming on $\mathcal{A} \cup \mathcal{B} \cup C$, the homomorphism $\bar{\theta}\left(h^{\prime}(\cdot)\right)$ is not a variable renaming either. Since $B=\mathcal{A} \cup \mathcal{B} \cup C$ is an f-block, $\bar{\theta}\left(h^{\prime}(\cdot)\right)$ can be extended to an endomorphism $h$ on $J$, which again is not a variable renaming on $\mathcal{A} \cup \mathcal{B} \cup C$. This contradicts our above observation that such an endomorphism cannot exist.

Property 3. There is no endomorphism on $J^{\prime}$ that maps a labeled null in $\mathcal{D}$ to a constant.

Proof of Property 3. Suppose to the contrary that there exists an endomorphism $h^{\prime}$ on $J^{\prime}$ that maps a labeled null in $\mathcal{D}$ to a constant. We define a mapping $h=\bar{\theta}\left(h^{\prime}(\theta(\cdot))\right)$. Clearly, $h$ is a chomomorphism from $J$ to $J$. By restricting $h$, we can consider $h$ also as a c-homomorphism from $\mathcal{A} \cup \mathcal{B} \cup \mathcal{C}$ to $J$. We claim that $h$ restricted to $\mathcal{A} \cup \mathcal{B} \cup C$ is in fact a homomorphism. To show this, consider an arbitrary constant $s$ occurring in some fact of $\mathcal{A} \cup \mathcal{B} \cup C$. We have to show that $h(s)=s$ holds. We distinguish two cases as to whether $s$ is equal to some $b_{i}$ from the local constants of $\mathcal{B}$ or not. First consider the case $s=b_{i}$ for some $i$. Then $\theta\left(b_{i}\right)=d_{i}$. Since $h^{\prime}$ is a homomorphism, we have $h^{\prime}\left(d_{i}\right)=d_{i}$. Finally, by the definition of $\bar{\theta}$, we have $\bar{\theta}\left(d_{i}\right)=b_{i}$. In total, we thus have $\bar{\theta}\left(h^{\prime}\left(\theta\left(b_{i}\right)\right)\right)=b_{i}$ as desired. Now consider the case $s \in \operatorname{const}(J) \backslash l \operatorname{cst}(\mathcal{B})$. In this case, we have $\theta(s)=s$. Since $h^{\prime}$ is a homomorphism, we also have $h^{\prime}(s)=s$. Finally, since $s \in \operatorname{const}(J)$, we also have $\bar{\theta}(s)=s$. In total, we thus again have $\bar{\theta}\left(h^{\prime}(\theta(s))\right)=s$ as desired.

By assumption, $h^{\prime}$ maps some null in $\mathcal{D}$ to a constant. By definition, $\theta$ maps each null in $\mathcal{B}$ to a null in $\mathcal{D}$. Hence, $h^{\prime}(\theta(\cdot))$ maps some null in $\mathcal{B}$ to a constant, and so does $h=\bar{\theta}\left(h^{\prime}(\theta(\cdot))\right)$. However, by the above considerations, this contradicts the assumption that $B=\mathcal{A} \cup \mathcal{B} \cup C$ is an f-block in $\operatorname{core}(\operatorname{chase}(I, \sigma))$. 
We are now ready to complete the proof of Claim 3: More specifically, we show that $\operatorname{core}\left(\operatorname{chase}\left(I^{\prime}, \sigma\right)\right)$ has f-block size at least $m+1$. Let $h^{\prime}$ be the endomorphism on $J^{\prime}$ that reduces $J^{\prime}$ to $\operatorname{core}\left(\operatorname{chase}\left(I^{\prime}, \sigma\right)\right)$. By Claim 1 , we know that $\mathcal{A} \cup \mathcal{B} \cup C \cup \mathcal{D}$ is connected. By Claim 2 and 3, no null in $\mathcal{A} \cup \mathcal{B} \cup C \cup \mathcal{D}$ is mapped to a constant by $h^{\prime}$. Hence, also $h^{\prime}(\mathcal{A} \cup \mathcal{B} \cup C \cup \mathcal{D})$ is connected. By Claim 2, $h^{\prime}$ is a variable renaming on $\mathcal{A} \cup \mathcal{B} \cup C$. Hence, $h^{\prime}(\mathcal{A} \cup \mathcal{B} \cup C)$ has the same size as $\mathcal{A} \cup \mathcal{B} \cup \mathcal{C}$, namely, $m$. Finally, we also know that $h^{\prime}(\mathcal{D})$ contains at least one fact $h^{\prime}(R[\vec{a}, \vec{d}]) \notin h^{\prime}(\mathcal{A} \cup \mathcal{B} \cup C)$ such that $h^{\prime}(R[\vec{a}, \vec{d}])$ is connected to $h^{\prime}(\mathcal{A} \cup \mathcal{B} \cup C)$. Thus, altogether, $h^{\prime}(\mathcal{A} \cup \mathcal{B} \cup C \cup \mathcal{D})$ is connected and has size at least $m+1$ in core $\left(\operatorname{chase}\left(I^{\prime}, \sigma\right)\right)$. This concludes the proof of Claim 3 and, hence, also of Theorem 4.10 .

We now also provide a detailed proof of Theorem 4.14:

THEOREM 4.14. The class of nested GLAV mappings has effective bounded anchor.

Proof. As before, by Lemma 4.5, it suffices to consider a single nested tgd $\sigma$. We have to show that there exists a recursive function $a$ that, for a given schema mapping specified by a nested $\operatorname{tgd} \sigma$, returns an integer $a(\sigma)$ witnessing bounded anchor for $\sigma$. Let $I$ be a source instance and let $J \subseteq \operatorname{core}(\operatorname{chase}(I, \sigma))$ be connected. We have to show that there exists a source instance $I^{\prime}$ with $\left|I^{\prime}\right| \leq a(\sigma)|J|$ and connected $J^{\prime} \subseteq \operatorname{core}\left(\operatorname{chase}\left(I^{\prime}, \sigma\right)\right)$ with $\left|J^{\prime}\right| \geq|J|$.

By Corollary 3.15, we know that the facts in the connected subinstance $J$ of core $(\operatorname{chase}(I, \sigma))$ must be generated by a single chase tree in the chase forest $\mathcal{F}_{\sigma, I}$, i.e., $J$ is generated by some chase tree $T_{p}$ with pattern $p$. Let $I_{p}$ be the canonical source instance of $p$. As in the proof of Theorem 4.10, it can be shown that also core $\left(\operatorname{chase}\left(I_{p}, \sigma\right)\right)$ contains a connected subinstance $J^{*}$ of size at least $|J|$. W.l.o.g., we may assume that $J^{*}$ is a maximal connected subinstance of $\operatorname{core}\left(\operatorname{chase}\left(I_{p}, \sigma\right)\right)$. Again by Corollary $3.15, J^{*}$ is actually a subinstance of the canonical target instance $J_{p}$ of pattern $p$.

Analogously to Lemma 3.22, let $k=v_{\sigma} w_{\sigma}+1$, where $v_{\sigma}$ is the number of existentially quantified variables (i.e., distinct Skolem functions) and $w_{\sigma}$ is the number of universally quantified variables in $\sigma$. Then, we define $a(\sigma)$ as the maximum size of the canonical source instance of any $k$-pattern of $\sigma$.

If $\left|I_{p}\right| \leq a(\sigma) \leq a(\sigma)|J|$, then we set $I^{\prime}=I_{p}$ and $J^{\prime}=J^{*}$ and we are done. Otherwise, $p$ cannot be a $k$-pattern. By Lemma 3.17, there exists a $k$-pattern $p_{0}$ with $p_{0} \leq_{k} p$, i.e., pattern $p$ is obtained from $p_{0}$ by a sequence of $k^{+}$-increments. More precisely, there exists a sequence of patterns $p_{0}, p_{1}, p_{2}, \ldots, p_{n}$ with $p_{n}=p$, such that each $p_{i}$ is obtained from $p_{i-1}$ by a adding a further clone of some subtree $t$ of $p_{i-1}$, such that $p_{i-1}$ already contains at least $k$ clones of $t$ and for every subtree $t^{\prime}$ of $t, p_{i-1}$ contains at most $k$ clones.

For $i \in\{0, \ldots, n-1\}$, let $J_{p_{i}}$ denote the canonical target instance of $p_{i}$ and define $J_{p_{i}}^{*}=J^{*} \cap J_{p_{i}}$. By the connectedness of $J^{*}$, also $J_{p_{n-1}}^{*}$ must be a connected subinstance of $J_{p_{n-1}}$, since adding another clone cannot make two disconnected subinstances connected. By an easy induction argument, we conclude that $J_{p_{i}}^{*}$ must be a connected subinstance of $J_{p_{i}}$ for every $i$.

Moreover, for every $i, J_{p_{i}}^{*}$ is (up to variable renaming) indeed a subinstance of $\operatorname{core}\left(\operatorname{chase}\left(I_{p_{i}}, \sigma\right)\right)$, i.e., every homomorphism $h$ from $J_{p_{i}}$ to $\operatorname{core}\left(\operatorname{chase}\left(I_{p_{i}}, \sigma\right)\right)$ is a null renaming on the nulls of $J_{p_{i}}^{*}$. The reason for this is that, otherwise, by Lemma $3.22, h$ can be extended to a homomorphism $h_{p}$ from $J_{p}$ to $\operatorname{core}\left(\operatorname{chase}\left(I_{p}, \sigma\right)\right)$, which is not a null renaming on $J^{*}$ either. But this contradicts the above assumption that $J^{*}$ is a maximal connected subinstance of $\operatorname{core}\left(\operatorname{chase}\left(I_{p}, \sigma\right)\right)$.

By the definition of $a(\sigma)$, we clearly have $\left|I_{p_{0}}\right| \leq a(\sigma)$. If $\left|J_{p_{0}}^{*}\right| \geq|J|$, then we are done with $I^{\prime}=I_{p_{0}}$ and $J^{\prime}=J_{p_{0}}^{*}$. Otherwise, our sequence of $k^{+}$-increment steps keeps adding clones so that eventually a pattern $p_{i}$ is reached for which $\left|J_{p_{i}}^{*}\right| \geq|J|$ holds. We thus set $I^{\prime}=I_{p_{i}}$ and $J^{\prime}=J_{p_{i}}^{*}$. It remains to show that $\left|I_{p_{i}}\right| \leq a(\sigma)|J|$ holds. To this end, we use the property shown in Lemma 3.17, that the 
sequence of $k^{+}$-increment steps adds clones in a "top-down" fashion, i.e., whenever a subtree $t$ is added, then all subtrees $t^{\prime}$ of $t$ have at most $k$ clones in $t$. In particular, this means that every $k^{+}$-increment step adds at most $a(\sigma)$ atoms to the source instance, i.e., $\left|I_{p_{i}} \backslash I_{p_{i-1}}\right| \leq a(\sigma)$ for every $i$. Moreover, the $k^{+}$-increment steps may be arranged in arbitrary order as long as supertrees are added before any subtrees of them are added. Hence, we may arrange the $k^{+}$-increment steps in such an order that subtrees containing at least one atom from $J^{*}$ are added before the subtrees that contain no such atom. In this way, it is guaranteed that at most $|J|-1$ increment steps are needed to reach a subinstance of size at least $|J|$. In total, this proves that the desired source instance $I_{p_{i}}$ with $\left|J_{p_{i}}^{*}\right| \geq|J|$ indeed satisfies the condition $\left|I_{p_{i}}\right| \leq a(\sigma)|J|$.

\section{B UNDECIDABILITY PROOFS FOR SECTION 5}

In this section, we give the correctness proof for Theorem 5.1, as well as the proof for Theorem 5.2. We start with the former:

THEOREM 5.1. It is undecidable whether a given plain SO tgd is equivalent to a GLAV mapping in the presence of a single source key dependency.

Proof. (Correctness). Having already shown the construction, we now proceed to showing the correctness of our reduction in two parts.

Part 1 (If TM does not halt, then $\sigma$ has unbounded f-block size). Assume that TM does not halt. To prove that $\sigma$ has unbounded f-block size, we show that for an arbitrary integer $K$, we can find a source instance $I$ such that the core of the canonical solution for $I$ under $\sigma$ has f-block size of at least $K$. In particular, we show that for a computation of length $n$ of TM, we can construct instance $I$ such that the core of the canonical universal solution has f-block size of at least $n$ (in actuality, at least $n^{2} / 2$, but the exact size is of no immediate concern here). Since a non-halting Turing machine can have computations of arbitrary length, it then follows that $\sigma$ has unbounded f-block size.

Let $C_{0}, C_{1}, \ldots, C_{n}$ denote the sequence of configurations of the run of TM with $n+1$ configurations, where we pad blanks so that for every $C_{i}$, we represent $n+1$ tape cells. That is, for each time instant $0 \leq x \leq n$ and tape position $0 \leq y \leq n$, we have tape symbol $a_{x y}$ and cursor information $c_{x y}$. We also have for every time instant $x$ the corresponding state $q_{x}$. Given such a run of TM, we now construct a corresponding source instance $I$. We first represent the tape atoms:

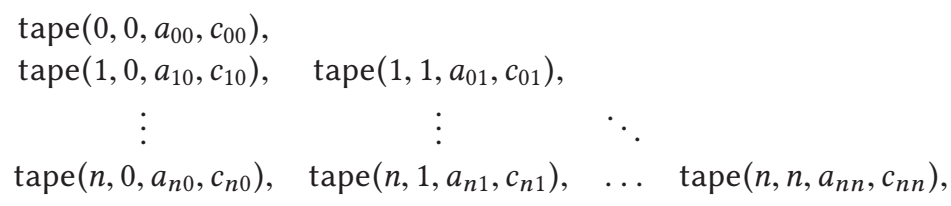

We now represent the rest of the atoms contained in $I$ :

$$
\begin{aligned}
& \text { state }\left(0, q_{0}\right), \text { state }\left(1, q_{1}\right), \ldots, \text { state }\left(n, q_{n}\right), \\
& Z(0), S(0,1), \ldots, S(n-1, n), \\
& A_{0}\left(a_{0}\right), \ldots, A_{k}\left(a_{k}\right), \quad Q_{0}\left(q_{0}\right), \ldots, Q_{l}\left(q_{l}\right), \quad C_{0}(0), C_{1}(1) .
\end{aligned}
$$

We now show that $J=\operatorname{chase}(I, \sigma)$ has f-block size at least $n$. Intuitively, the structure of $J$ can be seen in Figure 14.

Specifically, $J$ contains the following atoms:

$$
\begin{aligned}
& O(\hat{f}(0,0)), \\
& N(\hat{f}(0,0), \hat{f}(1,1)),
\end{aligned}
$$




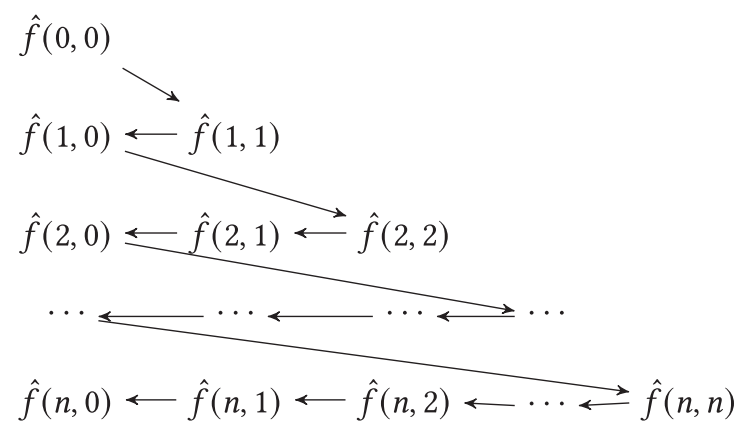

Fig. 14. Structure of a prototypical target instance.

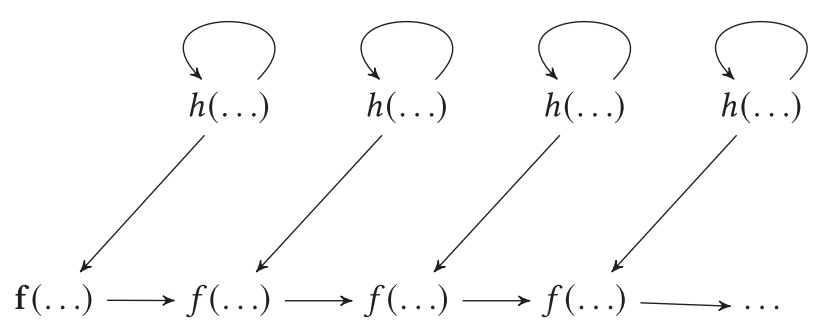

Fig. 15. Abstract representation of the structure of $J$. Edges denote $N$ facts. Bold font denotes the $O$ fact.

$$
\begin{aligned}
& N(\hat{f}(1,1), \hat{f}(1,0)), N(\hat{f}(1,0), \hat{f}(2,2)), \\
& N(\hat{f}(2,2), \hat{f}(2,1)), N(\hat{f}(2,1), \hat{f}(2,0)), N(\hat{f}(2,0), \hat{f}(3,3)), \\
& \ldots \\
& N(\hat{f}(n, n), \hat{f}(n, n-1)), \ldots, N(\hat{f}(n, 1), \hat{f}(n, 0)) .
\end{aligned}
$$

We will call these atoms $J_{b}$. Already the first $N$ atoms in each line of $J_{b}$ witness the claim of an f-block of size at least $n$, while the rest of each line shows that the atoms are indeed connected through a "chain" of nulls. Intuitively, these $N$ atoms contain $\hat{f}(i, j)$ nulls, which correspond to the contents of tape cell $j$ at time instant $i$. Each $N$ atom defines a connection between two cells, such that the whole configuration is enumerated.

What we have shown so far is that the canonical solution $J_{b}$ contains an f-block of size at least $n$. What remains to show is that $J_{b}$ is also part of the core $J^{*}=\operatorname{core}(J)$. First, observe that no guards fire on $I$. That is, apart from the atoms in $J_{b}, J$ only contains the following "self-loop" for each labeled null $X, N(X, X)$, as required by the unfoundedness trap.

We now show that $J$ is indeed a core $\left(J=J^{*}\right)$. In Figure 15, we give an abstract illustration of the structure of $J$. Let us call an $N$-fact $x$ the predecessor of an $N$-fact $y$, if-with $N$ considered as a graph-it is possible to reach $y$ from $x$. Towards a contradiction, assume that there exists a proper endomorphism mapping some $N$-fact $\eta$ to another $N$-fact $\eta^{\prime}$. Whenever this happens, also all predecessors of $\eta$ must be mapped to respective predecessors of $\eta^{\prime}$. However, this is not possible for the deepest predecessor, namely, the $N$-fact that has no predecessor: If $\eta^{\prime}$ precedes $\eta$, there are simply not enough predecessors of $\eta^{\prime}$, and if $\eta^{\prime}$ succeeds $\eta$, there is no fact connected to an $O$-fact in the chain. Also, the self-loops using the $h$ function symbol cannot be used as the images of our proper endomorphism, since they do not start with an $O$-fact. That is, no proper endomorphism of $J$ can exist. Therefore, $J$ is indeed a core and with that, $J_{b} \subseteq J$ witnesses an f-block of size at least $n$. Thus, follows the claim. 


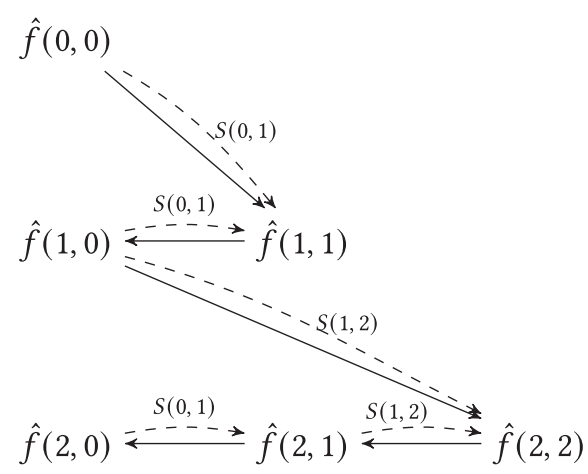

Fig. 16. Direction of $N$ relation (solid) and $S$ relation (dashed) edges.

Part 2 (If $\sigma$ has unbounded f-block size, then TM does not halt). Assume that $\sigma$ has unbounded f-block size. We then have to show that TM does not halt. We will prove this in two steps: First, we show that given that the size of the core of the universal solutions under $\sigma$ is unbounded, there is an $N$-path of unbounded length in some core of $\sigma$ (Claim A). Then, we show that if there exists an $N$-path of unbounded length in some core of $\sigma$, then TM has a computation of unbounded length (Claim B). We can then conclude that TM does not halt.

Let us first formalize some common constructs: We say that the size of the core under $\sigma$ is unbounded, if there is no integer $b$, such that for any source instance $I$, the number of atoms contained in $\operatorname{core}(\operatorname{chase}(I, \sigma))$ is at most $b$. We say that there exists an $N$-path of length $n$ in some core of $\sigma$, if there are distinct labeled nulls $x_{1}, x_{2}, \ldots, x_{n}$ such that the atoms $N\left(x_{1}, x_{2}\right), N\left(x_{2}, x_{3}\right), \ldots, N\left(x_{n-1}, x_{n}\right)$ are contained in core $(\operatorname{chase}(I, \Sigma))$ for some source instance $I$. We say that there exists an $N$-path of unbounded length in the core of $\sigma$, if for any integer $b$ there exists an $N$-path of length $b^{\prime} \geq b$ in some core of $\sigma$.

Claim A. If the size of the core under $\sigma$ is unbounded, then there exists an $N$-path of unbounded length in some core of $\sigma$.

We will show the following, which implies the claim: If the $S$-relation in the source instance $I$ has no "join" nodes (i.e., no nodes with more than one predecessor; in this case the $S$-relation forms a forest, where some of the roots are possibly not marked by $Z$ ), and the $N$-relation of core $(\operatorname{chase}(I, \sigma))$ has $k$ facts, then the $N$-relation of $\operatorname{core}(\operatorname{chase}(I, \sigma))$ consists of a founded simple path of size $k / 3$ and of $2 k / 3$ facts representing $k / 3$ "traps" of the form $N(\hat{h}(x, y), \hat{h}(x, y)), N(\hat{h}(x, y), \hat{f}(x, y))$, such that each node $\hat{h}(x, y)$ occurs in exactly two "trap" atoms and $\hat{f}(x, y)$ occurs in the simple path. We call this structure a $k$-gadget and illustrate it in Figure 17. The core of the universal solutions under $\sigma$ is always a $k$-gadget (for some $k$ ). Hence, if the $k$-gadget has unbounded size, so does the length of this founded simple path $(k / 3)$. Thus follows the original claim.

Proof of Claim A. Consider a vertex $\hat{f}(x, y)$ in the $N$-relation of chase $(I, \sigma)$.

Case 1: if $x=y$, then the unique predecessor of $\hat{f}(x, y)$ is $\hat{f}\left(x^{\prime}, 0\right)$, where $x^{\prime}$ is the unique predecessor of $x$ in the S-relation.

Case 2: if $x \neq y$, then the unique predecessor of $\hat{f}(x, y)$ is $\hat{f}\left(x, y^{\prime}\right)$, where $y^{\prime}$ is a successor of $y$ in the S-relation (see Figure 16 for an example of how the directions of the relations may look like in a concrete instance). Assume that $y$ is reachable from an element in $Z$. We will later discuss the situation of this not being the case. In principle, there could be several successors of $y$. In our extended example in Figure 18, consider $\hat{f}(2,1)$, which has as its two predecessors $\hat{f}(2,2)$ and 


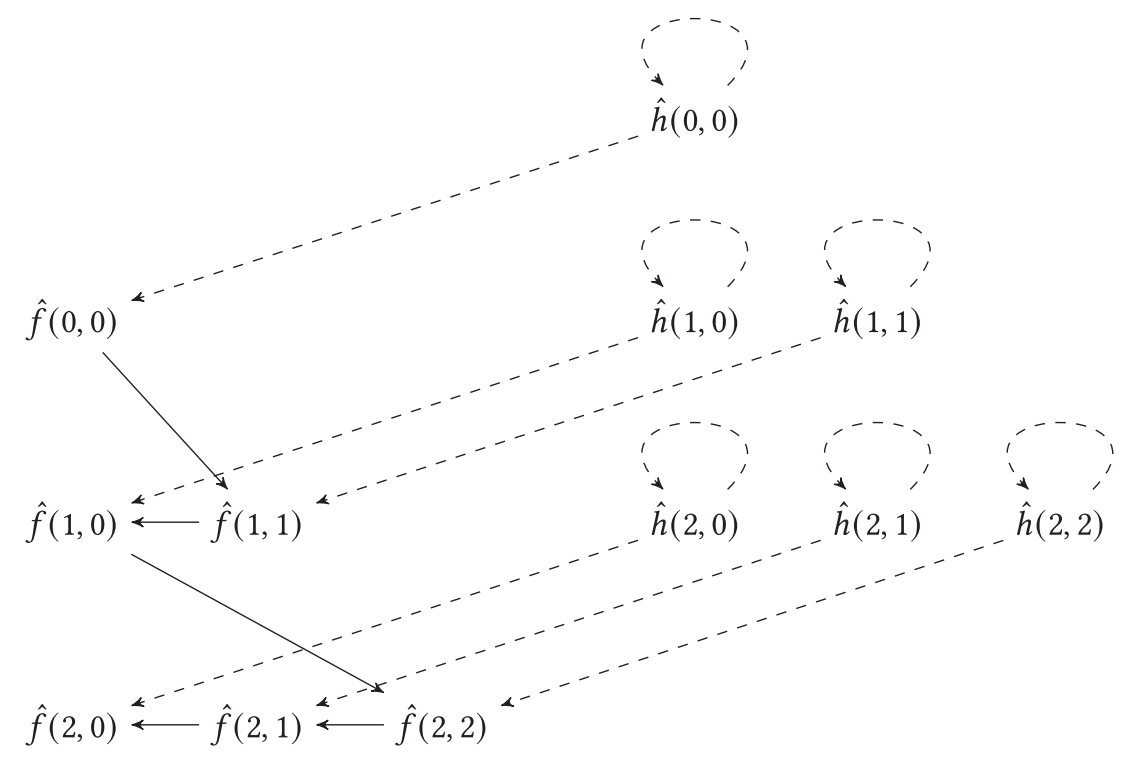

Fig. 17. $k$-gadget: edges denote the $N$ relation formed of $k$ atoms. Of these, $k / 3$ form a founded simple path (solid) and $2 k / 3$ are not part that founded simple path (dashed).

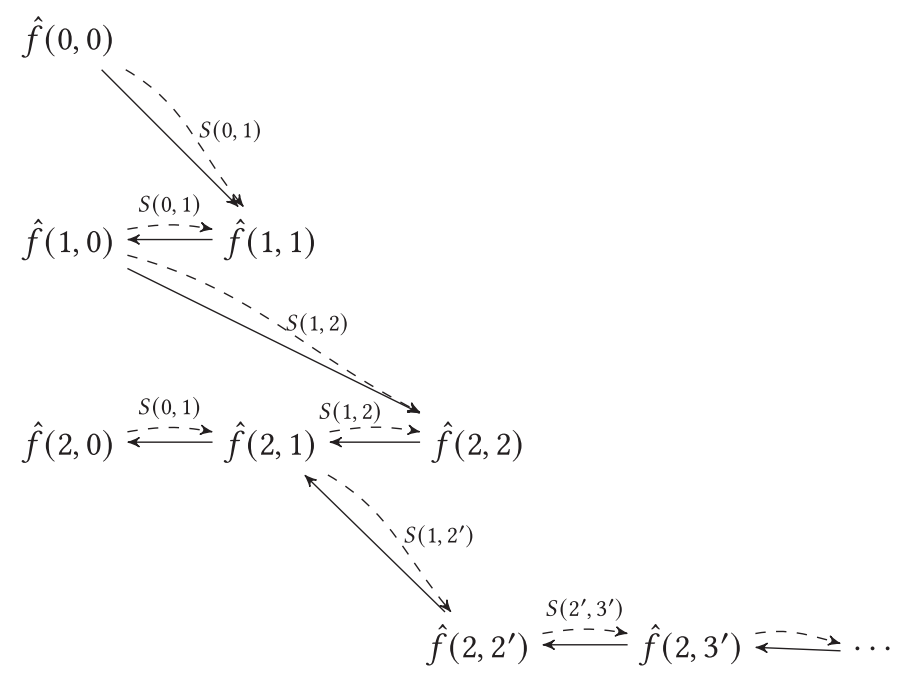

Fig. 18. The lower path splitting at $\hat{f}(2,1)$ must be unfounded. In particular, note that only the upper path has a diagonal $\hat{f}(2,2)$ after the split, while the lower one containing $\hat{f}\left(2,2^{\prime}\right)$ never reaches a diagonal after the split.

$\hat{f}\left(2,2^{\prime}\right)$. This corresponds to 1 having two successors in the S-relation, namely, 2 and $2^{\prime}$. However, note that $(x, y)$ is reached by horizontal arcs (directed from right to left) from the diagonal point $(x, x)$. In our example, this diagonal is already one of the predecessors, i.e., $(2,2)$. Hence, we have the inequalities $y<y^{\prime}<x$, where " $<$ " denotes the transitive closure of the S-relation. Note that there can be only one $S$-successor of $y$ from which $x$ is reachable, since, otherwise, we would have first a "fork" (i.e., a node with two or more successors) and then a "join" (i.e., a node with two or 


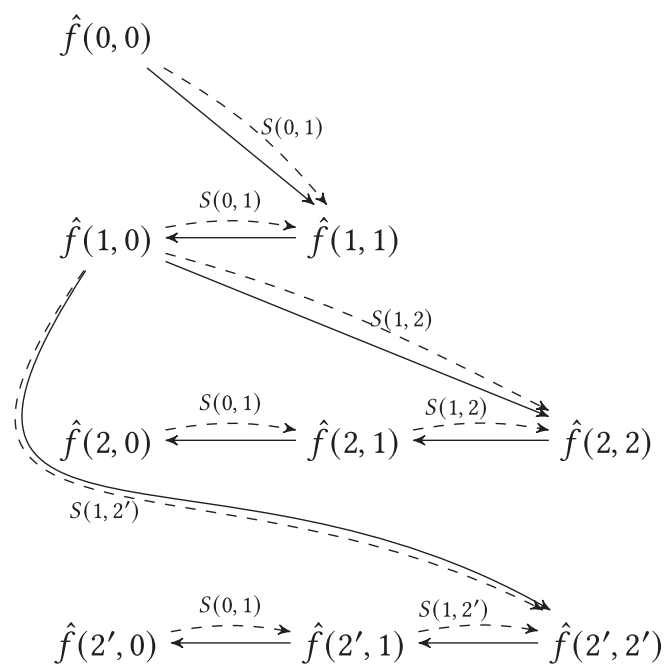

Fig. 19. Part of a canonical universal solution where a split occurs. In contrast to Figure 18 , note that the direction of the $N$-edge at the split is in the opposite direction, and the split occurs due to the first (time) argument of $\hat{f}(\cdot, \cdot)$. Thus, we do reach a diagonal in both split paths. Since the direction of the $N$-paths at the split are different, this split yields a tree.

more predecessors). Consider the $S$-successors of $y$ from which $x$ is not reachable. In our example, this is $2^{\prime}$. Each of these $S$-successors gives rise to a simple $N$-path leading to $\hat{f}(x, y)$, which we claim is not founded (i.e., cannot contain a node marked with $O$ ).

We now show that each node in the path connected to $\hat{f}(x, y)$ has the form $\hat{f}(x, z)$ for some $z$. Indeed, the only point where the first argument $v$ in $\hat{f}(v, w)$ changes is when $v=w$ (at the diagonal). In our example, this is $\hat{f}(2,2)$. However, by our assumption, $x$ is not reachable from $y$, so we can never come to a node $\hat{f}(x, x)$. In our example, we see this in the lower branch containing $\hat{f}\left(2,2^{\prime}\right)$, and we know that $2(x)$ is not reachable from $2(y)$. Moreover, it holds that $y<z$, therefore $z$ never coincides with the least element of the < order, thus we cannot reach the origin (by traversing the $S$ relation backwards). We thus know that $y^{\prime}$ is uniquely determined by $x$ and $y$ and, therefore, also $\hat{f}\left(x, y^{\prime}\right)$ is the unique $N$-predecessor of $\hat{f}(x, y)$, which is reachable from an origin (see Figure 18 for an intuitive illustration of the situation discussed in this paragraph).

We thus have shown that each vertex $\hat{f}(x, y)$ in the $N$-relation of $\operatorname{chase}(I, \sigma)$ has a unique predecessor reachable from an origin. Note that all predecessors not reachable from an origin cannot be part of the core, since they will be mapped to "traps." In particular, this includes the situation mentioned before in Case 2, where the assumption that $y$ is reachable from an element if $Z$ does not hold. See Figure 19 for an example of part of such a chase result. Therefore, $\operatorname{core}(\operatorname{chase}(I, \sigma))$ is a forest (plus traps connected to each non-origin node of the forest. Further note that all founded paths in the forest collapse to the longest founded path. We therefore have shown that the $N$-relation of core $(\operatorname{chase}(I, \sigma))$ can be decomposed into a founded simple path and a trap for each non-origin node.

Claim B. If there exists an $N$-path of unbounded length in some core of $\sigma$, then TM has a computation of unbounded length.

Proof Sketch of Claim B. Assume that $J$ contains a path of length $\hat{K}$. If $\hat{K} \geq \frac{n(n+1)}{2}+1$, then the source instance contains an encoding of a correct TM computation of length $n$. The formula is 
derived from the size of the lower triangle of the configuration matrix including the main diagonal, plus a single $O$ fact.

We proceed by induction on $\hat{K}$. We use as induction hypothesis that all entries of the configuration matrix corresponding to the path of length $i$ are correct (i.e., correspond to the actual configuration of the Turing machine).

For the base case, $i=2$, we know that no guard constraint with conclusion "fail" has fired and the guard constraints fully specify the first three configuration matrix entries (intuitively at $(0,0)$, $(1,0)$, and $(1,1))$. Thus, all entries of the configuration matrix up to that point are correct.

For the induction step, assume that all entries of the configuration matrix up to $i$ are correct. We then have to show that entry $i+1$ is also correct. Configuration matrix entry $i+1$ depends only on three specific entries of the configuration matrix. By construction of the enumeration, we know that the three configuration matrix entries that entry $i+1$ depends on precede entry $i+1$ in the enumeration. In particular, by the fact that $J$ contains only a single path defining that enumeration (alternate paths stemming from forks or joins are prohibited by source constraints and guards as shown in Claim A), these three entries in the configuration matrix are uniquely defined. Thus, by assumption, we know that they are correct. The guard constraints fully specify the configuration matrix entry $i+1$ by the fact that the Turing machine starts from the empty tape and thus only has a single possible run. Therefore, we know that also entry $i+1$ is correct.

THEOREM 5.2. It is undecidable whether a given plain SO tgd is equivalent to a nested GLAV mapping in the presence of a single source key dependency.

Proof. In this proof, we use the same Turing machine simulation technique as described previously. Namely, the given TM is simulated as a plain SO-tgd $\sigma$, which, when run on an intended source instance, produces a target instance whose core is a chain of binary $N$-facts, founded in an $O$-fact. It remains to show that $\sigma$ is logically equivalent to a nested GLAV mapping if and only if the simulated Turing machine halts on the empty input.

Suppose that TM does not halt. We show that in this case, the simulating plain SO-tgd $\sigma$ cannot be logically equivalent to any nested GLAV mapping. To this end, we recall several facts from the literature.

First, we recall the property of SO-tgds [11], stating that the chase with this class of dependencies always terminates and delivers a universal solution. Since nested tgds are SO-tgds, this property also holds for them. We further recall the concept of CQ-equivalence [9]. It follows from Proposition 3.5 in Reference [9] that for mappings specified by SO-tgds (and thus also for mappings specified by nested tgds) CQ-equivalence can be defined as follows: The mappings $\mathcal{M}_{1}, \mathcal{M}_{2}$ are CQ-equivalent iff for arbitrary source instance $I$, $\operatorname{core}\left(\operatorname{chase}\left(I, \mathcal{M}_{1}\right)\right) \cong \operatorname{core}\left(\operatorname{chase}\left(I, \mathcal{M}_{2}\right)\right)$ holds (where $\cong$ denotes isomorphism). By Proposition 3.6 in Reference [9], we know that logical equivalence implies CQ-equivalence and, hence, also the failure of CQ-equivalence implies the failure of logical equivalence. It thus suffices to show that for the mapping $\mathcal{M}_{\sigma}$ specified by the plain SO-tgd $\sigma$ simulating a non-halting TM, there exists a source instance $I$ such that for any nested GLAV mapping $\mathcal{M}$, core $\left(\operatorname{chase}\left(I, \mathcal{M}_{\sigma}\right)\right) \nsucceq \operatorname{core}(\operatorname{chase}(I, \mathcal{M}))$ holds.

Let $C$ be a class of "intended" source instances as described in the proof of Theorem 5.1, with a forest in the $S$ relation, in which all tree roots are marked by $Z$-facts. Assume that $C$ contains a family of instances $\left\{I_{1}, I_{2}, \ldots\right\}$, in which every $I_{n}$ contains a tree of depth $n$ in the $S$ relation. As shown in the proof of Theorem 5.1, for every $n$, core $\left(\operatorname{chase}\left(I_{n}, \sigma\right)\right)$ consists of a single f-block with f-degree at most three. This is because each fact in the $N$-chain shares nulls with at most two other $N$-facts belonging to the same chain, namely, with the predecessor and the successor facts. The 
initial $N$-fact of the chain is marked with an $O$-fact. Finally, every other fact of the $N$-chain shares a null with a single $N$-fact from a "self-loop trap." From the proof of Theorem 5.1 it is easy to see that the length of the $N$-chain and thus the f-block size of core $\left(\operatorname{chase}\left(I_{n}, \sigma\right)\right)$ is greater than $n$, and thus, we have that $\sigma$ has bounded f-degree and unbounded f-block size on $C$.

From Theorem 4.18, we know that any nested GLAV mapping has bounded f-degree on $C$ if and only if its f-block size on $C$ is bounded as well. Specifically, if $\mathcal{M}$ is a nested GLAV mapping, by Claim 2 of Lemma 4.19, we know that for each integer $k$, there exists an integer $m_{k}$, such that for arbitrary source instance $I$, if $\operatorname{core}(\operatorname{chase}(I, \mathcal{M}))$ has f-block size $b \geq m_{k}$, then it has f-degree $g \geq k$. Observe that, in our case, we have f-degree 3. Thus, we know that if $\operatorname{core}(\operatorname{chase}(I, \mathcal{M}))$ has f-block size $b \geq m_{3}$, then it has f-degree $g \geq 3$. Given such number $m_{3}$, it suffices to choose $n>m_{3}$ to ensure that f-block size of core $\left(\operatorname{chase}\left(I_{n}, \mathcal{M}_{\sigma}\right)\right)$ is greater than $m_{3}$ as well. But then, $\operatorname{core}\left(\operatorname{chase}\left(I_{n}, \mathcal{M}\right)\right) \nsubseteq \operatorname{core}\left(\operatorname{chase}\left(I_{n}, \mathcal{M}_{\sigma}\right)\right)$ holds, since we have seen above that $\mathrm{f}$-degree of $\mathcal{M}_{\sigma}$ is bounded by three on $C$, and $I_{n} \in C$. The failure of CQ-equivalence between $\mathcal{M}_{\sigma}$ and arbitrary nested GLAV mapping $\mathcal{M}$ is demonstrated, as required. As a consequence, we obtain that no nested GLAV mapping is logically equivalent to the mapping specified by $\sigma$, which simulates a nonhalting TM.

Now, suppose that the TM halts. In this case, core $\left(\operatorname{chase}\left(I, \mathcal{M}_{\sigma}\right)\right)$ has bounded f-block size, as shown in the proof of Theorem 5.1. But then, $\sigma$ is even logically equivalent to some GLAV mapping, according to Reference [28], Theorem 3.11.

Thus, we have shown that TM halts iff the mapping based on its simulating plain SO-tgd $\sigma$ is logically equivalent to a nested GLAV mapping. The claim of the theorem follows from the undecidability of the halting problem.

\section{PROOF OF LEMMAS 5.5 AND 5.7}

LEMMA 5.5. Let $K_{1}$ and $K_{2}$ be instances and let $\theta$ be a c-homomorphism from $K_{1}$ to $K_{2}$. Let $\Sigma$ be a set of egds and let $K_{1}^{\prime}$ denote an instance that is obtained from $K_{1}$ by a sequence $S_{1}$ of applications of egds from $\Sigma$. Then there exists a sequence $S_{2}$ of applications of egds from $\Sigma$ to instance $K_{2}$, transforming $K_{2}$ into instance $K_{2}^{\prime}$, such that there exists a c-homomorphism $\theta^{\prime}$ from $K_{1}^{\prime}$ to $K_{2}^{\prime}$. Moreover, whenever $S_{1}$ leads to the unification of two domain elements $s_{1}, s_{2} \in \operatorname{dom}\left(K_{1}\right)$, then also $S_{2}$ leads to the unification of $\theta\left(s_{1}\right), \theta\left(s_{2}\right) \in \operatorname{dom}\left(K_{2}\right)$.

Proof. Let $K_{1}^{0}, \ldots, K_{1}^{m}$ be the sequence of instances corresponding to the chase sequence $S_{1}$ where $m$ is the length of $S_{1}: K_{1}^{0}=K_{1}$ and $K_{1}^{m}=K_{1}^{\prime}$. For each $i \geq m, K_{1}^{i}$ is transformed into $K_{1}^{i+1}$ by a unification of two constants, which we denote $a^{i}, b^{i} \in \operatorname{dom}\left(K_{1}^{i}\right)$ such that $K_{1}^{i+1}=K_{1}^{i}\left[a^{i} \mapsto b^{i}\right]$.

We now recursively define a sequence of c-homomorphisms translating the instances produced by the chase of $K_{1}$ into instances produced in the chase of $K_{2}$. We define $\theta^{0}=\theta$ and $\theta^{i+1}=\left[\theta^{i}\left(a^{i}\right) \mapsto \theta^{i}\left(b^{i}\right)\right] \circ \theta^{i}$. Finally, we obtain a sequence $\left(K_{2}^{i}\right)$ of instances corresponding to the chase of $K_{2}$. We set $K_{2}=K_{2}^{0}$ and $K_{2}^{i+1}=K_{2}^{i}\left[\theta^{i}\left(a^{i}\right) \mapsto \theta^{i}\left(b^{i}\right)\right]$, for $0<i<m$. In total, we have

(1) a sequence of instances $\left(K_{1}^{0}=K_{1}\right), \ldots,\left(K_{1}^{m}=K_{1}^{\prime}\right)$,

(2) a sequence of constant pairs $\left(a^{0}, b^{0}\right), \ldots,\left(a^{m-1}, b^{m-1}\right)$ such that $K_{1}^{i+1}=K_{1}^{i}\left[a^{i} \mapsto b^{i}\right]$,

(3) a sequence of c-homomorphisms $\left(\theta^{0}=\theta\right), \ldots, \theta^{i+1}=\theta^{i} \circ\left[\theta^{i}\left(a^{i}\right) \mapsto \theta^{i}\left(b^{i}\right)\right]$,

(4) a sequence of instances $\left(K_{2}^{0}=K_{2}\right), \ldots, K_{2}^{i+1}=K_{2}^{i}\left[\theta^{i}\left(a^{i}\right) \mapsto \theta^{i}\left(b^{i}\right)\right]$.

By induction on $i$, we show that the instances $K_{2}^{0}, \ldots, K_{2}^{i}$ make up a beginning of a chase sequence of $K_{2}$ with $\Sigma$, in which the same egds as in $S_{1}$ are applied. (Note that it may happen that the equality $\theta^{i}\left(a^{i}\right)=\theta^{i}\left(b^{i}\right)$ already holds in $K_{2}^{i}$, in which case the substitution $\left[\theta^{i}\left(a^{i}\right) \mapsto \theta^{i}\left(b^{i}\right)\right]$ is void and $K_{2}^{i+1}=K_{2}^{i}$ ). Furthermore, we show that $\theta^{i}$ is indeed a c-homomorphism from $K_{1}^{i}$ to $K_{2}^{i}$. 
The base case $i=0$ is by the assumption of the lemma that $\theta=\theta^{0}$ is a c-homomorphism from $K_{1}=K_{1}^{0}$ to $K_{2}=K_{2}^{0}$, the corresponding chase sequence is empty.

Now assume that the claim holds for the first $i$ egd applications in $S_{1}$, namely, that $\theta^{i}$ is a chomomorphism from $K_{1}^{i}$ to $K_{2}^{i}$ and that $K_{2}^{i}$ can be obtained from $K_{2}$ via $i$ egd applications, where the same egds as in the sequence $S_{1}$ trigger. At step $i+1$, an egd $\tau=\forall \vec{x}\left(\phi(\vec{x}) \rightarrow x_{r}=x_{q}\right)$ triggers on $K_{1}^{i+1}$ with an assignment $\vec{d}$ for $\vec{x}$, in which the $r$ th element is $a^{i}$ and the $q$ th element is $b^{i}$ yielding the instance $K_{1}^{i+1}=K_{1}^{i}\left[a^{i} \mapsto b^{i}\right]$. Since $K_{2}^{i}=\theta^{i}\left(K_{1}^{i}\right)$ and $\phi(\vec{x})$ does not contain constants, $K_{2}^{i} \mid=$ $\phi\left(\theta^{i}(\vec{d})\right)$ and thus the same egd $\tau$ applied to $K_{2}^{i}$ yields the instance $K_{2}^{i+1}=K_{2}^{i}\left[\theta^{i}\left(a^{i}\right) \mapsto \theta^{i}\left(b^{i}\right)\right]=$ $\theta^{i+1}\left(K_{1}^{i}\right)=\theta^{i+1}\left(K_{1}^{i+1}\right)$, as desired.

At every step the substitution $a^{i} \mapsto b^{i}$ in $S_{1}$ corresponds to the substitution $\theta^{i}\left(a^{i}\right) \mapsto \theta^{i}\left(b^{i}\right)$ and that $\theta^{i}=\theta \circ \epsilon_{i}$ holds, where $\epsilon_{i}$ mimics the constant unifications in the chase sequence $S_{2}$, i.e., $\epsilon_{i}=\left[\theta^{i-1}\left(a^{i-1}\right) \mapsto \theta^{i-1}\left(b^{i-1}\right)\right] \circ \cdots \circ\left[\theta^{0}\left(a^{0}\right) \mapsto \theta^{0}\left(b^{0}\right)\right]$. The desired c-homomorphism $\theta^{\prime}$ from $K_{1}^{\prime}$ to $K_{2}^{\prime}$ is obtained as $\theta^{m}=\theta \circ \epsilon_{m}$, where $\epsilon_{m}$ mimics all unifications enforced by the sequence $S_{2}$ of applications of egds from $\Sigma$ to $K_{2}$.

Moreover, applying the substitution $\theta^{i}\left(a^{i}\right) \mapsto \theta^{i}\left(b^{i}\right)$ also enforces the equality $\theta\left(a^{i}\right)=\theta\left(b^{i}\right)$. Ultimately, the sequence $S_{2}$ of applications of egds from $\Sigma$ to $K_{2}$ results in the instance $K_{2}^{\prime}=K_{2}^{m}$, in which the equalities $\theta\left(a^{i}\right)=\theta\left(b^{i}\right)$ have been enforced for every $i \in\{0 \ldots m-1\}$. Note that $S_{1}$ may enforce further equalities by transitivity, e.g.: suppose that some $b^{i}$ and $b^{j}$ with $i \neq j$ are the same element from $\operatorname{dom}\left(K_{1}\right)$. Then $S_{1}$ also enforces the unification $a_{i}=a_{j}$. But then the analogous unifications (e.g., $\theta\left(a_{i}\right)=\theta\left(a_{j}\right)$ in our example) are of course also enforced by transitivity of the equalities due to sequence $S_{2}$.

LEMMA 5.7. Let $\sigma$ be a nested $\operatorname{tg} d$ and let $\Sigma_{s}$ be a set of source egds. Let $p$ be a $k$-pattern of $\sigma$ with $k \geq 2$ and consider two isomorphic (complete) subtrees $T_{1}$ and $T_{2}$ in $p$ such that the roots of $T_{1}$ and $T_{2}$ are siblings. Let $I_{p}$ denote the canonical source instance of $p$ and let $\vec{b}$ (respectively, $\vec{c}$ ) denote the local constants used in the variable instantiations in $T_{1}$ (respectively, in $T_{2}$ ) to construct $I_{p}$. Let $I_{p}^{s}$ denote the legal, canonical source instance of $p$ and let $\vec{b}^{\prime}$ (respectively, $\vec{c}^{\prime}$ ) denote the pseudolocal constants used in the variable instantiations in $T_{1}$ (respectively, in $T_{2}$ ) to construct $I_{p}^{s}$. We write $b_{i}^{\prime}$ (respectively, $\left.c_{i}^{\prime}\right)$ to refer to the ith component of $\vec{b}^{\prime}$ (respectively, $\vec{c}^{\prime}$ ). Then $\vec{b}^{\prime}$ and $\vec{c}^{\prime}$ fulfill the following properties:

(1) for all $i, j$, if $b_{i}^{\prime}=b_{j}^{\prime}$ in $I_{p}^{s}$, then also $c_{i}^{\prime}=c_{j}^{\prime}$ holds;

(2) for all $i, j$, if $b_{i}^{\prime}=c_{j}^{\prime}$ in $I_{p}^{s}$, then also $c_{i}^{\prime}=c_{j}^{\prime}$ holds.

Proof. Consider the canonical source instance $I_{p}$ of $p$ and let $\vec{b}$ (respectively, $\vec{c}$ ) denote the local constants used in the variable instantiations in $T_{1}$ (respectively, in $T_{2}$ ) to construct $I_{p}$. We write $b_{i}$ (respectively, $c_{i}$ ) to refer to the components of $\vec{b}$ (respectively, $\vec{c}$ ). Moreover, we write $\mathcal{B}$ (respectively, $C$ ) to denote the facts in $I_{p}$ obtained from instantiating the antecedents of the parts in $T_{1}$ (respectively, in $T_{2}$ ). Clearly, the facts in $\mathcal{B}$ (respectively, $C$ ) contain as arguments either local constants of $\mathcal{B}$ (respectively, $C$ ) or constants used for instantiating variables from the antecedents in parts at common ancestors of $T_{1}$ and $T_{2}$. Recall from Section 3 that there is a one-to-one correspondence between the parts in $T_{1}$ and $T_{2}$. In particular, there exists a c-isomorphism $\theta$ between $\mathcal{B}$ and $C$, such that $\theta\left(b_{i}\right)=c_{i}$ for every $i$. Moreover, $\theta$ can be extended to a c-homomorphism from $I_{p}$ to $I_{p} \backslash \mathcal{B}$ by setting $\theta(s)=s$ for all $s \notin \vec{b}$.

We now prove Property (1). Suppose that $b_{i}^{\prime}=b_{j}^{\prime}$, i.e.: there is a chase sequence $S_{1}$ with the egds in $\Sigma$, which transforms $I_{p}$ into $I_{p}^{s}$, s.t. $b_{i}$ and $b_{j}$ are unified. We have to show that the chase of $I_{p}$ with $\Sigma$ also unifies $c_{i}$ and $c_{j}$. By considering the c-homomorphism $\theta$ and applying Lemma 5.5 , we conclude that there exists a sequence $S_{2}$ of applications of egds from $\Sigma$ to transform $I_{p}$ 
into some instance $I^{*}$, s.t. there exists a homomorphism $\theta^{\prime}$ from $I_{p}^{s}$ to $I^{*}$. Moreover, whenever the sequence $S_{1}$ unifies elements $s_{1}$ and $s_{2}$ from $\operatorname{dom}\left(I_{p}\right)$, then $S_{2}$ unifies $\theta\left(s_{1}\right)$ and $\theta\left(s_{2}\right)$ from $\operatorname{dom}\left(\theta\left(I_{p}\right)\right)$. We are assuming that $b_{1}^{\prime}=b_{2}^{\prime}$ holds, i.e., the chase of $I_{p}$ with $\Sigma$ unifies $b_{1}$ and $b_{2}$. But then the chase of $\theta\left(I_{p}\right)$ with $\Sigma$ unifies $c_{1}=\theta\left(b_{1}\right)$ and $c_{2}=\theta\left(b_{2}\right)$. Note that $\theta\left(I_{p}\right) \subseteq I_{p}$. Hence, the sequence of egd firings in $\theta\left(I_{p}\right)$ is also applicable in $I_{p}$. Hence, the unification of $c_{1}$ and $c_{2}$ must also be enforced when we construct $I_{p}^{s}$ from $I_{p}$ via the chase with $\Sigma$, i.e., $c_{i}^{\prime}=c_{j}^{\prime}$ indeed holds in $I_{p}^{s}$.

To prove Property (2), we again consider the c-homomorphism $\theta$ and apply Lemma 5.5. Note that $\theta\left(b_{i}\right)=c_{i}$ and $\theta\left(c_{j}\right)=c_{j}$. By assumption, the sequence $S_{1}$ of egd applications to $I_{p}$ unifies the elements $s_{1}=b_{i}$ and $s_{2}=c_{j}$ from $\operatorname{dom}\left(I_{p}\right)$. Hence, the sequence $S_{2}$ of egd applications in $\theta\left(I_{p}\right)$ unifies $c_{i}$ and $c_{j}$. By $\theta\left(I_{p}\right) \subseteq I_{p}$, we conclude that the chase of $I_{p}$ with $\Sigma$ also unifies $c_{i}$ and $c_{j}$.

\section{REFERENCES}

[1] Marcelo Arenas, Pablo Barceló, Leonid Libkin, and Filip Murlak. 2014. Foundations of Data Exchange. Cambridge University Press, Cambridge, UK.

[2] Marcelo Arenas, Jorge Pérez, Juan Reutter, and Cristian Riveros. 2013. The language of plain SO-tgds: Composition, inversion, and structural properties. f. Comput. Syst. Sci. 79, 6 (2013), 763-784.

[3] Marcelo Arenas, Jorge Pérez, and Cristian Riveros. 2009. The recovery of a schema mapping: Bringing exchanged data back. ACM Trans. Datab. Syst. 34, 4 (2009), 22:1-22:48.

[4] Patricia C. Arocena, Boris Glavic, and Renee J. Miller. 2013. Value invention in data exchange. In Proceedings of the SIGMOD Conference. ACM, 157-168.

[5] Angela Bonifati, Elaine Qing Chang, Aks V. S. Lakshmanan, Terence Ho, and Rachel Pottinger. 2005. HePToX: Marrying XML and heterogeneity in your P2P databases. In Proceedings of the 31st International Conference on Very Large Data Bases. 1267-1270.

[6] AnHai Doan, Alon Halevy, and Zachary Ives. 2012. 11 - XML. In Principles of Data Integration. Morgan Kaufmann, Boston, MA, 291-323.

[7] Ronald Fagin and Phokion G. Kolaitis. 2012. Local transformations and conjunctive-query equivalence. In Proceedings of the Symposium on Principles of Database Systems. 179-190.

[8] Ronald Fagin, Phokion G. Kolaitis, Rene J. Miller, and Lucian Popa. 2005. Data exchange: Semantics and query answering. Theor. Comput. Sci. 336, 1 (2005), 89-124.

[9] Ronald Fagin, Phokion G. Kolaitis, Alan Nash, and Lucian Popa. 2008. Towards a theory of schema-mapping optimization. In Proceedings of the Symposium on Principles of Database Systems. 33-42.

[10] Ronald Fagin, Phokion G. Kolaitis, and Lucian Popa. 2005. Data exchange: Getting to the core. ACM Trans. Datab. Syst. 30, 1 (2005), 174-210.

[11] Ronald Fagin, Phokion G. Kolaitis, Lucian Popa, and Wang Chiew Tan. 2005. Composing schema mappings: Secondorder dependencies to the rescue. ACM Trans. Datab. Syst. 30, 4 (2005), 994-1055.

[12] Ingo Feinerer, Reinhard Pichler, Emanuel Sallinger, and Vadim Savenkov. 2015. On the undecidability of the equivalence of second-order tuple generating dependencies. Inf. Syst. 48 (2015), 113-129.

[13] Ariel Fuxman, Mauricio A. Hernández, C. T. Howard Ho, Renée J. Miller, Paolo Papotti, and Lucian Popa. 2006. Nested mappings: Schema mapping reloaded. In Proceedings of the International Conference on Very Large Data Bases. 67-78.

[14] Georg Gottlob, Reinhard Pichler, and Emanuel Sallinger. 2015. Function symbols in tuple-generating dependencies: Expressive power and computability. In Proceedings of the Symposium on Principles of Database Systems. 65-77.

[15] Rihan Hai and Christoph Quix. 2019. Rewriting of plain SO tgds into nested tgds. Proc. VLDB Endow. 12, 11 (2019), 1526-1538.

[16] Alon Y. Halevy, Zachary G. Ives, Peter Mork, and Igor Tatarinov. 2003. Piazza: Data management infrastructure for semantic web applications. In Proceedings of the World Wide Web Conference. ACM, 556-567.

[17] Pavol Hell and Jaroslav Nešetřil. 1992. The core of a graph. Disc. Math. 109 (1992), 117-126.

[18] Mauricio A. Hernández, Howard Ho, Lucian Popa, Ariel Fuxman, Renée J. Miller, Takeshi Fukuda, and Paolo Papotti. 2007. Creating nested mappings with Clio. In Proceedings of the IEEE International Conference on Data Engineering. 1487-1488.

[19] Phokion G. Kolaitis, Maurizio Lenzerini, and Nicole Schweikardt (Eds.). 2013. Data Exchange, Integration, and Streams. Dagstuhl Follow-Ups, Vol. 5. Schloss Dagstuhl - Leibniz-Zentrum für Informatik.

[20] Phokion G. Kolaitis, Reinhard Pichler, Emanuel Sallinger, and Vadim Savenkov. 2014. Nested dependencies: Structure and reasoning. In Proceedings of the Symposium on Principles of Database Systems. 176-187.

[21] Phokion G. Kolaitis, Reinhard Pichler, Emanuel Sallinger, and Vadim Savenkov. 2018. Limits of schema mappings. Theor. Comput. Syst. 62, 4 (2018), 899-940. 
[22] Maurizio Lenzerini. 2002. Data integration: A theoretical perspective. In Proceedings of the Symposium on Principles of Database Systems. 233-246.

[23] Leonid Libkin. 2004. Elements of Finite Model Theory. Springer.

[24] Jayant Madhavan and Alon Y. Halevy. 2003. Composing mappings among data sources. In Proceedings of the International Conference on Very Large Data Bases. 572-583.

[25] Reinhard Pichler, Emanuel Sallinger, and Vadim Savenkov. 2013. Relaxed notions of schema mapping equivalence revisited. Theor. Comput. Syst. 52, 3 (2013), 483-541.

[26] Alessandro Raffio, Daniele Braga, Stefano Ceri, Paolo Papotti, and Mauricio A. Hernández. 2008. Clip: A visual language for explicit schema mappings. In Proceedings of the IEEE International Conference on Data Engineering. 30-39.

[27] Emanuel Sallinger. 2013. Reasoning about schema mappings. In Data Exchange, Information, and Streams. Dagstuhl Follow-Ups, Vol. 5. Schloss Dagstuhl - Leibniz-Zentrum fuer Informatik, 97-127.

[28] Balder ten Cate and Phokion G. Kolaitis. 2009. Structural characterizations of schema-mapping languages. In Proceedings of the International Conference on Database Theory. 63-72.

Received December 2018; revised September 2019; accepted October 2019 